IS-T-57|

RECEIVED BY TIC MAR 201973

\title{
A SYSTEMATIC STUDY OF THE PHOTODISINTEGRATION OF GERMANIUM ISOTOPES
}

Ph.D. Thes is Submitted to lowa State University,
February, 1973

J. J. McCarthy

\begin{abstract}
This report was prepared as an account of work sponsored by the United States Government. Neither the United States nor the United States Atomic Energy Commission, nor any of their employees, nor any of their contractors, subcontractors, or their employees, makes any warranty, express or implied, or assumes any legal liability or responsibility for the accuracy, completeness or usefulness of any information, apparatus, product or process disclosed, or represents that its use would not infringe privately owned rights.
\end{abstract}

Ames Laboratory, USAEC lowa State University

Ames, lowa 50010

Date of Manuscript: February, 1973 


\section{DISCLAIMER}

This report was prepared as an account of work sponsored by an agency of the United States Government. Neither the United States Government nor any agency Thereof, nor any of their employees, makes any warranty, express or implied, or assumes any legal liability or responsibility for the accuracy, completeness, or usefulness of any information, apparatus, product, or process disclosed, or represents that its use would not infringe privately owned rights. Reference herein to any specific commercial product, process, or service by trade name, trademark, manufacturer, or otherwise does not necessarily constitute or imply its endorsement, recommendation, or favoring by the United States Government or any agency thereof. The views and opinions of authors expressed herein do not necessarily state or reflect those of the United States Government or any agency thereof. 


\section{DISCLAIMER}

Portions of this document may be illegible in electronic image products. Images are produced from the best available original document. 


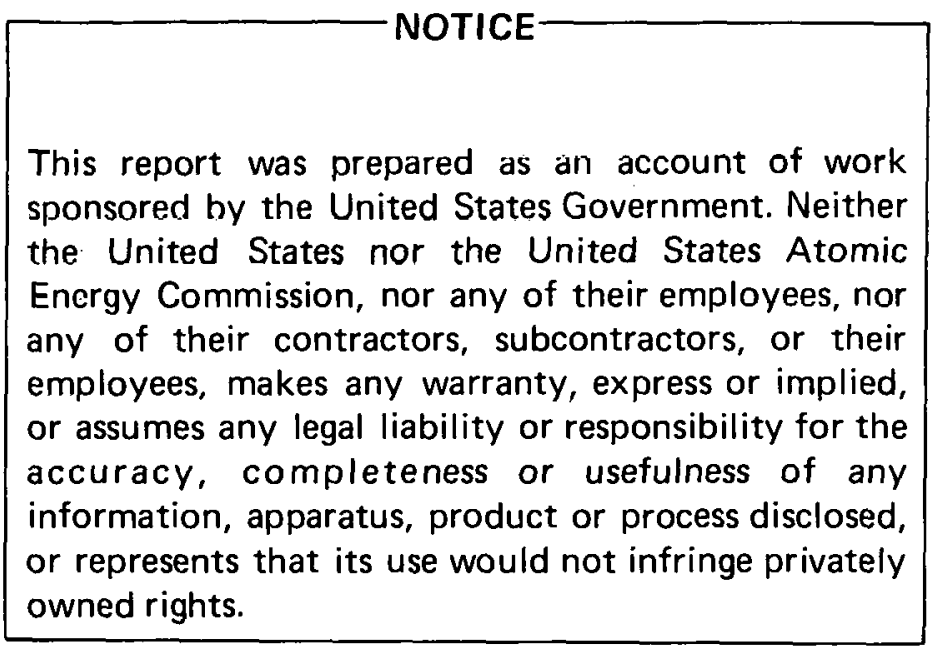

Available from: National Technical Information Service Department A

Springfield, VA 22151

Price: Microfictie $\$ 0.95$ 
A systematic study of the photodisintegration of. germanium isotopes

by

Jon Jay MeCarthy

A Dissertation Submitted to the

Graduate Faculty in Partial Fulfillment of

The Requirements for the Degree of

DOCTOR OF PHILOSOPHY

Department: Physics

Major: Nuclear Physics

Approved:

R. C. Morrison

In Charge of Major Work

For the Major Department

For the Graduate College

lowa State University

Ames, lowa

1973 


\section{ABSTRACT}

CHAPTER I. INTRODUCTION

Page

vi

The Giant Dipole Resonance I

Motivation for this Experiment 5

Systematic Study of Germanium 9

$\begin{array}{ll}\text { Experimental Considerations } & 10\end{array}$

CHAPTER 11. REVIEW OF THEORY 11.913

$\begin{array}{ll}\text { The Hydrodynamic Model } & 15\end{array}$

The Independent Particle Model 16

Isospin Considerations $\quad 18$

Implications for Germanium 23

CHAPTER 111 . EXPERIMENTAL INVESTIGATION 28

$\begin{array}{ll}\text { Activation Technique } & 28\end{array}$

Experimental Apparatus 29

Calibrations and Accuracy $\quad 34$

Data Collection $\quad 40$

CHAPTER IV. DATA ANALYSIS 43

Relation of Cross Section to Yield 43

Identification of Residual Activities 47

Reduction of Raw Data $\quad 49$

Calculation of Reduced Yield Curves 52

Extraction of Cross Sectlons $\quad 55$

Determination of Absolute Cross Sections 57 
CHAPTER V. EXPERIMENTAL RESULTS

Cross Section Results

Sources of Error

Comparison to Other Germanium Data

Total Absorption Cross Sections

80

CHAPTER VI. DISCUSSION AND CONCLUSIONS

Isospin Effects

Systematic Effects in the Total Cross Sections 90

Suggestions for Future Work 105

REFERENCES 
A systematic study of the photodisintegration

of germanium isotopes

Jon Jay McCarthy

Under the supervision of R. C. Morrison and D. J. Zaffarano

From the Department of Physics

lowa State University

Cross sections for the reactions ${ }^{70} \mathrm{Ge}(\gamma, n){ }^{69} \mathrm{Ge},{ }^{76} \mathrm{Ge}(\gamma, n){ }^{75} \mathrm{Ge}$, ${ }^{74} \mathrm{Ge}(\gamma, p){ }^{73} \mathrm{Ga},{ }^{70} \mathrm{Ge}(\gamma, \mathrm{np}){ }^{68} \mathrm{Ga},{ }^{72} \mathrm{Ge}(\gamma, \mathrm{np}){ }^{70} \mathrm{Ga},{ }^{74} \mathrm{Ge}(\gamma, \mathrm{np}){ }^{72} \mathrm{Ga}$, and ${ }^{76} \mathrm{Ge}(\gamma, \mathrm{np})^{74} \mathrm{Ga}$ have been measured from threshold to $40 \mathrm{MeV}$. Cross sections were extracted from bremsstrahlung Induced yield curves of the residual radloactlvity using least structure analysis. Cross sections measured in this experiment have been combined with the $(\gamma, n)$ and $(\gamma, 2 n)$ cross sections for ${ }^{70} \mathrm{Ge},{ }^{72} \mathrm{Ge},{ }^{74} \mathrm{Ge}$ and ${ }^{76} \mathrm{Ge}$ to form total absorption cross sections. The $(\gamma, n)$ and $(\gamma, 2 n)$ cross sections were measured in a companion experiment also performed at this Laboratory.

The total absorption cross sections for all isotopes display two prominent peaks. Peak energy and resonance width as well as peak and Integrated cross sections are presented for each isotope. The primary resonance has a peak energy, width and integrated cross section which Identify it as the usual giant dipole resonance. The secondary resonance has a peak energy at about $33 \mathrm{MeV}$ in ${ }^{70} \mathrm{Ge}$, whlch decreases systematically to about $27 \mathrm{MeV}$ in ${ }^{76} \mathrm{Ge}$. The observed second resonance has a strength nearly half of the giant resonance and a larger width than the giant 
resonance. Isospin effects in the primary resonance are identified and compared to the predictions of isospin formalism. Present models are not able to adequately describe the systematic character of the secondary resonance. Experimental evidence is presented to suggest that the secondary resonance is present over a wide range of nuclear mass number and may very pusslbly be a general property of nuclear matter. 


\section{CHAPTER I. INTRODUCTION}

The Giant Dipole Resonance

The dominant feature of the photon absorption cross section for nuclei is the giant dipole resonance. It is a large maximum, 3 to $10 \mathrm{MeV}$ in width located between 13 and $16 \mathrm{MeV}$ for medium and heavy elements, and near $20 \mathrm{MeV}$ for light nuclei. The giant dipole resonance is observed in the photodisintegration of all nuclei and may be viewed as a general property of nuclear matter.

Many features of the giant resonances of heavy elements have been described in terms of a purely collective model. In 1948, M. Goldhaber and E. Teller (1) first presented a model of collective nuclear vibrations resulting from electric dipole excitation. In this model the nucleus is assumed to consist of interpenetrating neutron and proton fluids of constant total density confined in a rigid boundary at the nuclear surface. The giant resonance is then associated with the lowest mode of the electric dipole counter-oscillations of these two fluids. The resonance frequency is determined by the dimensions, shape, and mass of the nucleus. In light nuclei, the giant resonance is most easily understood as the absorption of a photon to excite a single nucleon from its ground state configuration to a highly excited state, prior to de-excitation by particle ejection or by a radiative transition to the ground state. This approach to describing the giant resonance is the basis of the independent particle model proposed by Wilkinson (2). 
In either the collective or single particle descriptions of the giant resonance, it is often a convenient simplification to consider the neutron and proton as alternative states of one entity, the nucleon. I sobaric spin formalism has been developed to provide a mathematical framework for this concept. Since the electric dipole operator for photons can be expressed as a vector in isospin space, isospin formalism may be of some importance in describing photonuclear processes in both collective and independent particle models.

The isobaric spin formalism, also called isotopic spin or simply isospin, was first developed by Wigner (3) in 1937. The formalism was not generally applied to medium and heavy nuclei until recently.' It was always assumed that the strong coulomb potential in heavier nuclei would produce configuration mixing and destroy the isospin symmetry. However, the discovery of isobaric analog resonances in $(p, n)$ reactions in medium weight nuclei in the last decade (4) suggest that isospin is applicable in the heavier mass nuclei.

Three gross parameters can be determined from photonuclear cross section measurements; the cross section magnitude, the giant resonance energy, and its width. Photoneutron cross section measurements have long been studied, and the results of early studies by Baldwin and Klaiber (5) on a series of light and heavy elements provided the motivation for the Goldhaber Teller collective model. In more recent times, several groups have made extensive studies of $(\gamma, n)$ reactions for medium and heavy nuclei $(6,7)$ and give results for resonance energy and width in good agreement with collective models. The only structure allowed by the 
collective model is a gross splitting of the glant resonance into two resonances corresponding to oscillations along the major and $\mathrm{mlnor}$ axes of a deformed nucleus. Experimental studies of deformed nuclel, particularly ${ }^{235} \mathrm{U}(8),{ }^{153} \mathrm{Eu}(9),{ }^{160} \mathrm{Gd}(9)$ and ${ }^{181} \mathrm{Er}$ (10) have found st ructure In the giant resonance in good agreement with these collective mode predictions. Experimental measurements on light elements have yielded quite different results.

In light elements the glant resonance has been observed consistently at energles near $20 \mathrm{MeV}$. Experiments on oxygen in the 1950's by Katz and Montalbetti $(11)$ and others $(12,13)$ hinted at discrete levels in the low energy reglon. Later studies (14) confirmed the presence of the discrete levels in the oxygen cross section. Since that time many studies of light nuclel have been carried out. The structure seen in the cross sectlon varies from the very smooth shape of carbon (15) to the detailed structure of many discrete resonances as found in sillicon (16). An adequate description of the gross properties in the glant resonances for light nuclel has been embodied by a shell model description of single particle transitions.

Most experimental studies of the photonuclear giant resonance employ one of three general methods. These methods are total absorption measurements, direct measurements of the spectra of emitted particles, and activation measurements. Each method has limitations peculiar to the type of measurement.

Total absorption work is limited by the fact that nuclear absorption cross sections are at most $10 \%$ of the total absorption, the remainder being atomic absorption. The atomic absorption cross section must be 
well known in order to extract meaningful nuclear data from the measured total. Also since atomic processes vary as $z^{2}$ and $z^{4}$, while the nuclear absorption varies as NZ/A, data for high $Z$ elements are most difficult to analyze. Another limitation is that the result of this measurement is the total nuclear absorption, and offers no information about branching ratios to the various end products. Information on the partial reaction cross sections offers detailed information about the decay of the dipole state, and would facilitate evaluation of theoretical descriptions of this decay.

Measurements may be made of the energy spectra of emitted particles. This is a useful technique in light nuclei, where proton emission is strong, and the density of states in the residual nucleus is low. For heavler nuclei, neutron emission dominates and the final state density is high. Such experiments are hard to interpret. Often in this type of measurement it is difficult to separate competing reaction channels involving emission of the same particle, or multiple emission of that particle.

In activation work, the partial cross section for production of a particular daughter nucleus is measured by counting the radioactive decays of the product nuclei. The characteristics of the decay radiation provide a convenient basis for separating several reactions and their competing decay modes from multi-isotopic targets. This method provides specific information about the decay of the dipole state. However, this method will not work on reactions which lead to stable daughter nuclei. The activation method is also limited by the statistical inaccuracies 
resulting from the finite number of counts acquired. This makes reactions which result in decay half lives which are very short or very long extremely difficult to perform.

\section{Motivation for this Experiment}

While collective models describe many features of the giant resonance of very heavy nuclei, and single particle models apply well to light nuclei, the description of other nuclei often contains elements of both models.

Some features of light nuclei can be described by collective models and vise-versa. If it is fair to generalize at all, one can only say that a complete description of the giant resonance in any nucleus requires blending features of both models as the situation requires.

In 1957 D. M. Brink (17) showed that the shell model wave function of the state reached by the nuclear photo-effect contains the collective vibration described by Goldhaber and Teller. More recently V. V. Balashov (18) has pointed out that the particle-hole, or nucleon-nucleon correlations necessary to correct the independent particle model giant resonance predictions play an important role in collectivizing the theory.

It appears that the introduction of nucleon-nucleon correlations into elther model is necessary if the model is to describe correctly anything more than the gross features of the photonuclear cross section. A decision regarding the validity of one model or another is difficult. One uses the model which yields the most accurate predictions. 
The experimentalist aids the process of describing the photodisintegration of nuclei by measuring quantities that will test the predictions of various models. As noted earlier, experimental results played roles in prompting and verifying the collective and independent particle models. At the present time, as model calculations have become more detalled, experiments designed to study systematic effects over large and small ranges of $A, Z$ and $N$ are required to test predicted systematic variations of gross properties of the nuclear photo-effect. To date, such systematic studies have been few in number. Some examples exist in the studies of photoneutron cross sections in heavy nuclei by Fultz et al. (6), Bowman et al. (7), Berman et al. (9), and by Johnson at this laboratory (19). Comparisons of systematic studies by different laboratories are often hindered by the differences in normalization techniques.

Systematic studies in medium heavy nuclei have rarely been undertaken. The situation is exemplified by experiments designed to study isospin effects in these nuclei. In recent years, isospin formalism has been applied to medium heavy nuclei. This was prompted in part by the desire to explain structure found in the giant resonance of these nuclei.

Electric dipole absorption by a target nucleus with isospin $T_{0}\left(=T_{z}=(N-Z) / 2\right)$ leads to a giant resonance made up of two components with isospin $T_{<}=T_{0}$ and $T_{>}=T_{0}+1$. The two components will be split in energy and contain different fractions of the total dipole strength. Since single nucleons have $t=1 / 2$, and $t_{3}= \pm 1 / 2$, the $T_{<}$dipole state can decay by either proton or neutron emission. Proton emission from the $T_{>}$state is fully allowed, while neutron emission directly to the ground 
state of the daughter nucleus $\left(T_{0}^{D}=T_{0}^{P}-1 / 2=T_{>}-3 / 2\right)$ is isospin forbidden.

Experimental efforts have been made to observe this splitting of the giant resonance and verify the predicted strength ratio. Since neutron emission is isospin allowed from $T_{<}$states, but not from $T_{>}$states, directly to the residual ground state, while proton emission from both $T$ states is allowed, a direct comparison of $(\gamma, p)$ and $(\gamma, n)$ cross sections in the same nucleus should verify the splitting (20). However, varying normalizations of cross section results make these comparisons rather difficult.

Several attempts have been made to directly measure the $T_{>}$component of the giant resonance in the $(\gamma, p)$ and $(p, \gamma)$ reactions. Axel et al. (21) observed a sharp resonance in the ${ }^{89} Y(p, \gamma)$ cross section about 5 MeV above the $(\gamma, \mathrm{n})$ giant resonance, in good agreement with the energy splitting predicted by Akyuz and Fallieros (22). However the strength of the resonance was much less than that predicted for the $T_{;}$component. Shoda et al. (23) have measured the $\left(\gamma, p_{0}\right)$ cross sections for ${ }^{88} \mathrm{si}$ and ${ }^{90} \mathrm{Zr}$. Distinct resonances at 4-5 MeV above the $(\gamma, n)$ resonance $(24)$ were observed, but again the strengths were less than predicted. A possible explanation for the lack of strength in the proton channel is the existence of alternate isospin allowed decay channels, e.g. neutron emission to $T_{0} \pm 1 / 2$ exclted states of the residual nucleus.

A recent photon scattering experiment (25) (which reflects the total absorption cross section) has identified a structure 3-5 MeV above the glant resonance for a group of nuclei in the range $48 \leq A \leq 120$. 
The angular distribution of the scattered radiation excludes the possibility that it is electric quadrupole, and the strengths are close to isospin predictions.

The scattering data lends support to the reports of $(\gamma, n)$ cross sections for nickel and tin. Min (26) has pointed out that isospin effects are probably responsible for the difference in magnitudes of the ${ }^{58} \mathrm{Ni}$ and ${ }^{60} \mathrm{Ni}(\gamma, n)$ cross sections. In tin, structure above the giant resonance has been reported by fultz et al. (27), but isospin effects are clouded by the fact that the E2 giant resonance and the $T_{>}$giant resonance coincide in energy for this mass region.

A series of experiments on ${ }^{64} \mathrm{Zn}$ by Schamber (28), Paul, Amann, and Snover (29), and most recently Clark (30) have studied Isospin effects in this nucleus. Clark used his $(\gamma, p)$ cross section and the $(\gamma, n)$, $(\gamma, \mathrm{np}),(\gamma, 2 \mathrm{n})$ of Schamber to prepare a total absorption cross section for ${ }^{64} \mathrm{Zn}$. His values of $3 \mathrm{MeV}$ for the energy splitting and 0.2 for the ratio of $\mathrm{T}_{>}$strength to $\mathrm{T}_{<}$strength agree quite well with theoretical predictions.

The difficulty in interpreting the results of experiments measuring only partial reaction channels has pointed to the need for experimental investigations that provide information about all major decay channels, as in the case of ${ }^{64} \mathrm{Zn}$. Such experiments would provide a test of predictions of the isospin formalism for medium weight nuclei. They would provide a basis for attempts to develop a complete description of the giant resonance over the entire range of $A$. Systematic studies of giant resonance structures in medium heavy nuclei would help to explain the 
transition from the independent particle nature of light nuclei to the collective structure of the heavy nuclei. It is this need which is the motivation for the present study of germanium isotopes. This experiment is one of a series of experiments which will provide total absorption cross sections. for four germanium isotopes.

Systematic Study of Germanium

The plan of the entire series of experiments is shown in Table 1.

Table 1. Plan of experiments. Reactions measured in this experiment are denoted by the letter $A$. Reactions measured by direct counting of emitted nucleons are denoted by the letter $D$. $P$ is used to denote reactions that can be measured by $(p, \gamma)$ or $(\gamma, p)$ studies.

\begin{tabular}{ccccc}
\hline Reaction & ${ }^{70} \mathrm{Ge}$ & ${ }^{72} \mathrm{Ge}$ & ${ }^{74} \mathrm{Ge}$ & ${ }^{76} \mathrm{Ge}$ \\
\hline$\gamma, \mathrm{n}$ & $\mathrm{A}, \mathrm{D}$ & $\mathrm{D}$ & $\mathrm{D}$ & $\mathrm{A}, \mathrm{D}$ \\
$\gamma, \mathrm{P}$ & $\mathrm{P}$ & $\mathrm{P}$ & $\mathrm{A}$ & \\
$\gamma, \mathrm{nP}$ & $\mathrm{A}$ & $\mathrm{A}$ & $\mathrm{A}$ & $\mathrm{A}$ \\
$\gamma, 2 \mathrm{n}$ & $\mathrm{D}$ & $\mathrm{D}$ & $\mathrm{D}$ & $\mathrm{D}$ \\
\hline
\end{tabular}

The $(\gamma, n)$ and $(\gamma, 2 n)$ cross sections for all isotopes have been measured by H. Vander Molen (31) using direct neutron counting and separated isotopes of germanium as targets. The targets were exposed to bremsstrahlung from the lowa State University $70 \mathrm{MeV}$ Electron Synchrotron, and photoneutrons were detected by a high-efficiency, neutron-sensitive, scintillation detector. 
The $(\gamma, \mathrm{p})$ reactions in $70,72 \mathrm{Ge}$ lead to stable end products, ${ }^{69} \mathrm{Ga}$ and ${ }^{71} \mathrm{Ga}$, and cannot be measured by the activation method. These reactions could be studied by direct photoproton measurements with separated Ge targets. Results from $(p, \gamma)$ reactions using ${ }^{69} \mathrm{Ga}$ and ${ }^{71} \mathrm{Ga}$ targets could be used by applying the principle of detailed balance.

The present experiment is a study of the $(\gamma, n),(\gamma, p)$ and $(\gamma, n p)$ reactions in the four germanium isotopes which can be measured by the activation method. These reactions are labeled with an $A$ in Table 1. Samples were irradiated with bremsstrahlung and the residual activity of all daughter nuclei counted with $\mathrm{Ge}(L i)$ detectors. Partial reaction cross sections were separated by identifying the gamma activity by energy and half-life. The experiment was performed with targets of pure, natural germanium.

\section{Experimental Considerations}

As an activation experiment, this experiment is unique in one aspect. For the first time it has been possible in a single experiment to measure simultaneously and separately seven reactions from a multi-isotopic target. This fact is further dramatized by the wide range of half lives of the decaying daughter nuclei. The half lives for each decay are given In Table 2. They range from $8 \mathrm{~min}$, to $40 \mathrm{hr}$. In the past, separation of a maximum of four or five reactions was possible in activation studies, and then only if the daughter nuclei had convenient, widely separated, half lives. These problems are overcome by recording gamma decay spectra, eliminating the need to fit the combined decay curve of the daughter 
activity and perform a complicated statistical separation based on the half lives to obtain yields.

Table 2. Decay of reaction products

\begin{tabular}{|c|c|c|}
\hline Reactions & Mode of Decay & Reaction End Product \\
\hline${ }^{76} \mathrm{Ge}(\gamma, n){ }^{75} \mathrm{Ge}$ & $80.1 \mathrm{~min}$. & ${ }^{76}$ As \\
\hline${ }^{70} 0_{\mathrm{Ge}}(\gamma, n)^{69}{ }_{\mathrm{Ge}}$ & $\begin{array}{c}\beta^{+} \\
39.1 \mathrm{hr} .\end{array}$ & $69_{\mathrm{Ga}}$ \\
\hline${ }^{74} \mathrm{Ge}(\gamma, p){ }^{73} \mathrm{Ga}$ & $\begin{array}{c}8^{-} \\
4.8 \mathrm{hr} .\end{array}$ & ${ }^{73} \mathrm{Ge}$ \\
\hline${ }^{76} \mathrm{Ge}(\gamma, \mathrm{np}){ }^{74} \mathrm{Ga}$ & $\begin{array}{c}\beta^{-} \\
7.9 \mathrm{~min} .\end{array}$ & $74 \mathrm{Ge}$ \\
\hline${ }^{74} \mathrm{Ge}(\gamma, \mathrm{np}){ }^{72} \mathrm{Ga}$ & $\begin{array}{c}\beta^{-} \\
14.1 \mathrm{hr} .\end{array}$ & ${ }^{72} \mathrm{Ge}$ \\
\hline${ }^{72} \mathrm{Ge}(\gamma, \mathrm{np}){ }^{70} \mathrm{Ga}$ & $\begin{array}{c}\beta- \\
21.1 \mathrm{~min} .\end{array}$ & ${ }^{70} \mathrm{Ge}$ \\
\hline${ }^{70} \mathrm{Ge}(\gamma, \mathrm{np}){ }^{68_{\mathrm{Ga}}}$ & $\begin{array}{c}\beta+ \\
68.2 \mathrm{~min} .\end{array}$ & $68_{\mathrm{Zn}}$ \\
\hline
\end{tabular}

It is well to note that the advent of high efficiency, high resoIution $\mathrm{Ge}(\mathrm{Li})$ detectors has made it possible to separate the gamma spectra of the daughter nuclei with high precision.

The successful application of this new technique to the activation method is Important to photonuclear studies. With the high efficiency $\mathrm{Ge}(\mathrm{Li})$ detectors currently avallable many activation studies not possible in the past can now be performed with relative ease. 
In Chapter 11 we review theoretical models of the giant resonance and expand our discussion of isospin formalism. Chapter 111 describes the actual experimental investigation, and Chapter IV gives the details of the data analysis procedure. Cross section results are presented and compared to previous measurements on germanium in Chapter $V$. Total cross sections based upon the collected results are also presented in Chapter V. In the last chapter these results are discussed in the light of theory and experiment, and conclusions based on the present data are proposed. 


\section{CHAPTER II. REVIEW OF THEORY}

This chapter presents a brief review of several well-known models of the giant resonance. In this manner we hope to form a basis for a comparison with experimental results. In the first two sections, only the very important aspects of these models are presented. The third section includes a more detailed account of recent predictions of isospin calculations. The last section is devoted to exploring the implications of the various theories to the specific case of the germanium isotopes. One main feature of the giant dipole resonance is independent of the speclfic model chosen. This model independent feature is cast in the form of a sum rule for the dipole operator.

The dipole sum rule is a conservation law which gives the total integrated absorption cross section. In atomic systems it is the wellknown Thomas-Reiche-Kuhn (TRK) dipole sum rule, and is a consequence of commutation relations. The extension of the TRK sum rule to a nucleus of $Z$ protons and $N$ neutrons was first treated by J. S. Levinger and $H$. $A$. Bethe (32). The sum rule has been treated by many others, but the result remains essentially the same, and is commonly referred to as the classical dipole sum. The integrated absorption cross section into the state $|k\rangle$ is given by

$$
\int \sigma_{k}(E) d E=\frac{2 \pi^{2} e^{2} \hbar}{M c} \cdot f_{k}
$$

where $M$ is the mass of the particle undergoing the transition to level $k$. $f_{k}$ is the osclllator strength for a transition from the ground state $|0\rangle$ 
to an exclted state $|k\rangle$ defined by

$$
f_{k}=\frac{2 M E_{k}}{\hbar^{2}}|\langle k|z| 0\rangle|^{2}
$$

It can be shown that the sum over all states $k$, yields

$$
\sum_{k} \cdot f_{k}=\frac{-M}{\hbar 2}|\langle 0|[[H, z], z]| 0\rangle|^{2}
$$

For an electron in an atom, the Hamiltonian is

$$
H=p^{2} / 2 M+V(r)
$$

and

$$
\sum_{k} f_{k}=z
$$

just the TRR result. For the nucleus, the sum is modified to account for the effect of the recoiling nucleus by using the concept of effective charge. In terms of the modified sum of the oscillator strength the nuclear absorption cross section becomes

$$
\int \sigma(E) d E=\frac{2 \pi^{2} e^{2} h}{M c}\left[\sum_{i=1}^{Z}\left(\frac{N}{A}\right)^{2} f_{i}+\sum_{j=1}^{N}(-Z / A)^{2} f_{j}\right]
$$

or

$$
\int \sigma(E) d E=\frac{2 \pi^{2} e^{2} h}{M c} \cdot \frac{N Z}{A} .
$$

This integrated electric dipole absorption cross section has then a minimum value resulting from the kinetic energy term of the nuclear Hamiltonian. The sum is augmented further by potential energy terms 
which do not commute with the dipole operator. In the following, the factor by which the dipole sum exceeds its classical value will be called B so that:

$$
\int \sigma(E) d E=\frac{2 \pi^{2} e^{2} n}{M c} \beta \frac{N Z}{A}=0.06 \frac{N Z}{A} B \text { MeV barns. }
$$

Experimental values of the integrated cross section to $30 \mathrm{MeV}$ indicate that for the lightest elements, only about half the dipole sum is in the glant resonance. In heavy nuclel the cross section increases to become approximately one dipole sum.

The Hydrodynamic Model

Since Goldhaber and Teller first proposed a collective description of the dipole excltation (1), many authors have elaborated upon this concept. The theory has developed to the point that descriptions of glant resonance structure due to deformation and osclilations of the nuclear surface are avallable. This body of theory is known as the hydrodynamic model. Extensions of the simple collective model to include the nuclear symmetry energy and coulomb interactions have been effective in describing the giant resonance energy $E_{0}$ and predict the appearance of dipole overtone and quadrupole resonances at $2.86 \mathrm{E}_{\mathrm{o}}$ and $1.6 \mathrm{E}_{\mathrm{o}}$ respectively. The resonance energy is given by

$$
E_{0}=\frac{2.08}{R}\left[\frac{8 K N 2}{M A^{2}}\right]^{1 / 2}
$$

where 2.08 is the solution to the eigenvalue equation for a sphere of radius $R$, and $K$ is the constant coefficient of the symmetry energy in 
the seml-emplrical mass formula. A reasonable cholce of $K$ and $R$ lead to the result that

$$
E_{0}=80 \mathrm{~A}^{-1 / 3} \mathrm{MeV}
$$

This expression gives energies in good agreement to experimental values for the heavy nuclel.

The simple collectlve plcture falls to properly describe the width of the giant resonance. Experimentally, the presence of dipole overtones or the glant quadrupole resonance has not been established.

In recent years, recognition that the quadrupole oscillation of the nuclear surface could couple with the glant resonance has led to an expansion of the hydrodynamic theory called the Dynamic Collective Model. Calculations and estimates of both photonuclear absorption and scattering cross sections have been made by Le Tourneaux (33), Danos and Greiner (34) and others. The magnitude of the effects depends upon the softness of the nuclear surface; the parameters entering the calculations being transition rates and the energies of the low lying collective states. The predicted energies of the structure in the giant resonance agree reasonably well with experimental results for as light a nucleus as ${ }^{75}$ As (35), but calculated strengths often disagree with experimental values.

The Independent Particle Model

An independent partlcle model of the nuclear photo-effect has been emphasized by Wilkinson (2). In this treatment the giant resonance is ascribed to the electric dipole absorption of photons by the valence nucleons and those in the uppermost filled shell. These particles make transitions upward to the next unfilled shell of opposite parity 
according to the selectlon fule $\Delta l= \pm 1$. Wilkinson showed that dipole transitions from the last filled shell which are allowed by this selection rule go to levels sufficlently close together in energy to form a giant resonance. Furthermore, essentially all of the dipole oscillation strength is exhausted in transitions from the last filled shell levels to virtual shell levels in the continuum.

While independent particle model calculations are able to account for the existence of a glant resonance, a roughly correct oscillator strength, and a reasonable width $(\Gamma)$ for the glant resonance, the glant resonance energy predicted is far too low. The model gives a resonance energy as essentlally $41 \mathrm{~A}^{-1 / 3} \mathrm{MeV}$, the harmonic oscillator level spacing. We have al ready seen that the expression, $E_{0}=80 A^{-1 / 3}$, applies well to the heavy elements while light elements have their glant resonances near $20 \mathrm{MeV}$. The latter energy is still greater than the oscillator spacing. The introduction of particle-hole calculations by Elliott and Flowers (36) made substantial improvements in this matter of energy prediction.

The vital point is that the states which make up the giant resonance differ from the ground state in that one particle has been elevated to the next open shell of opposite parity. This particle is strongly correlated to the corresponding hole created in the excitation. Particlehole interactions cause configuration mixing and the resulting dipole state energy is a linear combination of the single particle-single hole energies. Furthermore, this interaction causes the dipole strength to be concentrated at the highest excitation energies. For a nucleus $A$, 
the energies of the hole states are found in the levels of nucleus A-1, and the particle state energies in nucleus $A+1$. The harmonic oscillator energles are replaced by ones closer to those which occur in the nucleus. Whlle these energies are not quite correct because nuclear core states are different in the $A-1, A+1$, and $A$ systems, the energies obtained are a much closer approximation to the true transition energies. Particlehole calculations for light nuclei have been quite successful in predictIng resonance energies, but still require drastic changes to properly alter the calculated strength to the point where it agrees with experiment. The observed strength in the glant resonance of light nuclei is approximately one-half the classical dipole sum.

\section{Isospin Considerations}

Isospin has some consequence for photonuclear reactions. The basic idea is simple. If one ignores the mass and charge differences of the proton and neutron, they can be considered collectively as a nucleon which can exist in two different states. Angular momentum formalism can be used to describe these states by assigning an isospin of $T=1 / 2$ to the nucleon with a $z$ component of $t_{z}=+1 / 2$ for neutrons and $t_{z}=-1 / 2$ for protons. The isospin of a nucleus $w i$ th $N$ neutrons and $Z$ protons is determined by addltion of the isospin vectors of the individual nucleons. Thus, for a particular nucleus, the $z$ component, $T_{z}$, is constant and equal to $1 / 2(\mathrm{~N}-\mathrm{Z})$. The total nuclear isospin can have a value from $1 / 2(\mathrm{~N}+\mathrm{Z})$ to $1 / 2(\mathrm{~N}-\mathrm{Z}):$ the nuclear ground state is assumed to possess the lowest possible value. A sequence of corresponding levels in neighboring isobars with the same $T$ but different values of $T_{z}$ is called 
an isobaric multiplet. The individual states in a multiplet are sald to be isobaric analogs of one another.

The electric dipole operator can be expanded in isospin space as:

$$
\begin{aligned}
D & =e \cdot \sum_{i=1}^{Z} r(i) \\
& =e \sum_{i=1}^{A} r(i)\left[1 / 2-t_{z}(i)\right] \\
& =\frac{e}{2} \sum_{i=1}^{A} r(i)-e\left[r(1) t_{z}(t)\right.
\end{aligned}
$$

where $t_{z}$ is an operator with elgenvalue $-1 / 2$ for protons and $+1 / 2$ for neutrons, and we have used the fact that the photon wavelength is large with respect to the nuclear dimensions. The first term is responsible for Thomson scattering; the second term induces electric dipole transitions. The cross section for forming an excited state with total isospin $T$ from a nuclear ground state $\left|T_{0} T_{0}\right\rangle$ is:

$$
\sigma\left(T_{0} \rightarrow T\right)=\frac{4 \pi^{2} e^{2}}{3 \pi c} E\left|\left\langle T_{0}\left|D_{3}\right| T_{0} T_{0}\right\rangle\right|^{2} .
$$

The selection rules for this process are $\Delta T=0, \pm 1,0 \neq 0$ as first demonstrated for self-confugate nuclei by Trainor (37) and later extended to the general case by Radicati (38) and Gell-Mann and Telegdi (39). Conservation of the $z$ component ellminates $\Delta T=-1$, thus the giant dipole resonance possesses two parts, one with states of isospin $T_{<}=T_{0}$ and another with states of isospin $T_{>}=T+1$, where $T_{0}$ is the isospin of the ground state. Application of the Wigner-Eckart gheorem to the matrlx 
element the above equation provides an estlmate of the relative strengths of the two components. If the magnitude of the reduced matrix elements were equal, geometric factors alone would determine the ratio of relative strengths as:

$$
\frac{\sigma\left(T_{0}+T_{0}+1\right)}{\sigma\left(T_{0}+T_{0}\right)}=\frac{1}{T_{0}} \quad \frac{E_{T_{0}}+1}{E_{T_{0}}}
$$

\section{Energy splitting}

Experimental observation of the two isospin components of the giant resonance is possible because there is an energy difference between the two parts. The center of strength of a resonance is defined as:

$$
\bar{E}=\frac{\int \sigma d E}{\int(\sigma / E) d E}
$$

and the energy splitting due to isospin effect is

$$
E=E_{>}-\bar{E}_{<}
$$

Several authors have estimated this energy splitting. A rough estimate has been derived by Akyuz and Fallleros (22);

$$
E=(60 \mathrm{MeV})(T+1) / \mathrm{A} .
$$

Leonardi has given a much more complicated formula (40): 


$$
\begin{aligned}
E & =\frac{T+1}{A} 2 \bar{E}\left(\frac{1-\alpha}{\alpha}\right)\left[1+\frac{2(1-T)}{\alpha A}-\frac{4 T}{\alpha^{2} A^{2}}\right]^{-1} \\
& \equiv \frac{T+1}{A} U
\end{aligned}
$$

where

$$
\alpha=\frac{3 \beta}{2 M E}\left(\left\langle R_{p}{ }^{2}\right\rangle-\frac{N \varepsilon}{2 T}\right)^{-1},\left\langle R_{p}{ }^{2}\right\rangle^{1 / 2} \text { is the r.m.s. proton }
$$

radius with

$$
\varepsilon=\left\langle R_{n}^{2}\right\rangle-\left\langle R_{p}^{2}\right\rangle,\left\langle R_{n}^{2}\right\rangle^{1 / 2} \text { is the r.m.s. neut ron radius. }
$$

The parameter a takes into account two body correlations in the nuclear ground state, and $\beta$ indicates by how much the integrated cross section exceeds the classical dipole sum. In spite of the complicated dependence on $T, \alpha$, and $A, U$ is essentially 55 to $70 \mathrm{MeV}$, except for light nuclei and nuclei with very large neutron excess.

Paul, Amann, and Snover (29) have fit the experimental data for a mass range near $A=60$ to an expression of the form:

$$
E=67 \mathrm{MeV}(1-39 \mathrm{~T} / \mathrm{A})(\mathrm{T}+1) / \mathrm{A} \text {. }
$$

\section{Sum rule calculations}

A useful quantity for model calculations is the bremsstrahlung welghted cross section defined by:

$$
\sigma_{-1} \equiv \int \sigma / E \mathrm{dE}
$$

A special sum rule first derived by Goulard and Fallieros (41) has been extended to the general case by $0^{\prime}$ Connell (42) and Hayward et al. 
(43). Thus sum rule relates the bremsitrahlung weighted cross section of the $T_{>}$component of the giant resonance to the total bremsstrahlung welghted cross section.

$$
\frac{\sigma_{-1}\left(T_{>}\right)}{\sigma_{-1}}=\frac{1}{T+1}\left(1-\frac{1.97\left\langle R_{p}^{2}\right) T(1-n)}{A^{4 / 3}}\right),
$$

where

$$
\eta=\frac{(2 T-1) A^{2 / 3}}{3 N Z}
$$

and $\left\langle R_{p}{ }^{2}\right\rangle$ is the mean square charge radius of the nucleus. The factor $n$ accounts for increased strength in the $T_{>}$channel as a result of ground state correlations. The factor in brackets is always less than one and is called the dynamic factor.

Leonardl has also given an expression for a sum rule result (40). Leonardl finds the ratio of the $T_{>}$bremsstrahlung weighted cross section to that of the $\mathrm{T}_{<}$to be:

$$
\frac{\sigma-1\left(T_{>}\right)}{\sigma-1}\left(T_{e}\right)=\frac{1}{T} \frac{\left(\frac{\alpha A}{2}-T\right)}{\left(\frac{\alpha A}{2}+1\right)}
$$

where

$$
\alpha \equiv \frac{3}{2} \frac{\beta}{M E}\left(\left\langle R_{p}^{2}\right)-\frac{N E}{2 T}\right)^{-1}
$$

is defined as before.

Experimental evidence for Isospin splitting and sum rule strengths is not overpowering. Verification of isospin effects can be provided in 
only one way, by observing all decay channels, and comparing reaction strengths in the varlous energy regions with predlctions which use the Isospin selection rules. Few nuclei have been studied In such detail. One noteworthy exception is ${ }^{64} \mathrm{Zn}$. Clark (30) has analyzed the total photoabsorption cross section for ${ }^{64} \mathrm{Zn}$ and found it in agreement with isospin predictions. Clark measured the $(\gamma, p)$ cross section for ${ }^{64} \mathrm{Zn}$, and found distinct resonances at energies of 16, 19 and $22 \mathrm{MeV}$. The peaks at 16 and $19 \mathrm{MeV}$ are identified as the $T_{<}$and $T_{>}$components of the giant resonance. These energies correspond well to a splitting seen by Schamber $(28)$ in the $(\gamma, n)$ channel. These values give an energy splittlng of $3 \mathrm{MeV}$ and ${ }^{64} \mathrm{Zn}$ in good agreement to. the value of $2.8 \mathrm{MeV}$ predicted by Goulard and Fallleros (41) and Paul et al. (29) and also $3.2 \mathrm{MeV}$ by Leonardl (40). Using the experlmental cross sectlons, clark has calculated the values for $\sigma_{-1}\left(T_{<}\right)$and $\sigma_{-1}\left(T_{>}\right)$. The ratio of these strengths in the combined $(\gamma, n),(\gamma, p)$ channels is 0.2 , which agrees quite well with the value 0.26 calculated by 0 'Connell (42).

\section{Implications for Germanium}

The application of theoretical model predictions to the four germanium isotopes studied in this experiment is straight forward.

In Table 3, the predictions of the hydrodynamic model for the energies of the giant resonance $E_{0}$, the first dipole overtone $E_{0}{ }^{\prime}$, and the quadrupole giant resonance $E_{2}{ }^{Q}$ are given for each isotope.

Table $4 a$ presents the energy of both isospin components of the giant resonance. The $T_{<}$energy has been chosen as $17 \mathrm{MeV}$, a value that agrees well with experimental giant resonance energies for the surrounding nuclei. 
Table 3. Predictions of the hydrodynamic model for overtone and giant quadrupole resonance energy ${ }^{a}$

Nucleus $\quad E_{0} \quad E_{0}{ }^{1} \quad E_{2}{ }^{Q}$

${ }^{70} \mathrm{Ge}$

19.7

55.5

31.1

${ }^{72} \mathrm{Ge}$

19.2

54.9

30.7

$74 \mathrm{Ge}$

19.0

54.3

30.4

${ }^{76} \mathrm{Ge}$

18.9

53.9

30.1

${ }^{a}$ All energles are in MeV.

Table 4a. Energies of the $T_{<}$and $T_{>}$giant resonance components ${ }^{a}$

$\begin{array}{llll}\text { Nucleus } & E_{T_{<}} & \text {Fallieros } & { }_{T_{T}} \\ \text { Leonardi } & \text { Paul }\end{array}$

$\begin{array}{lllll}{ }^{70} \mathrm{Ge} & 17 & 20.4 & 20.8 & 20.4 \\ { }^{72} \mathrm{Ge} & 17 & 21.2 & 21.0 & 20.5 \\ { }^{74} \mathrm{Ge} & 17 & 21.9 & 22.4 & 20.9 \\ { }^{76} \mathrm{Ge} & 17 & 22.5 & 22.6 & 21.3\end{array}$

a All energles are in MeV. 
The $T$, energies are found by adding the spllttings predicted by various authors to the $T_{<}$energy. Table $4 \mathrm{~b}$ presents the relative strengths of the two components as expressed by the ratio of bremsstrahlung weighted cross sections as calculated by Hayward et al. (43) and Leonardi (40).

Table 4b. Relative strengths of the $T$ components

$$
\sigma_{-1}\left(T_{>}\right) / \sigma_{-1}\left(T_{<}\right)
$$

Nucleus Leonardi

Hayward

\begin{tabular}{lcc}
\hline $70 \mathrm{Ge}$ & 0.24 & 0.23 \\
${ }^{72} \mathrm{Ge}$ & 0.17 & 0.13 \\
${ }^{74} \mathrm{Ge}$ & 0.13 & 0.09 \\
$76_{\mathrm{Ge}}$ & 0.10 & 0.05 \\
\hline
\end{tabular}

Since the isospin formalism has been successfully applied in the case of ${ }^{64} \mathrm{Zn}$, these predictions may serve as a useful tool in evaluating the germanium cross sections.

The partial reaction cross sections for germanium assume an added importance by consldering isospin selection rules. A diagram showing the isospin allowed transitions in the decay of the giant resonance of a typical nucleus is shown in figure 1.

Proton decay from both giant resonance states to the ${ }^{A-1} \times:{ }_{N}^{Z-1}$ ground state is allowed, as is neutron decay from the $T_{<}$state to the ground state of ${ }^{A-1} \times \underset{N-1}{Z}$. The lowest exclted state of proper isospin 


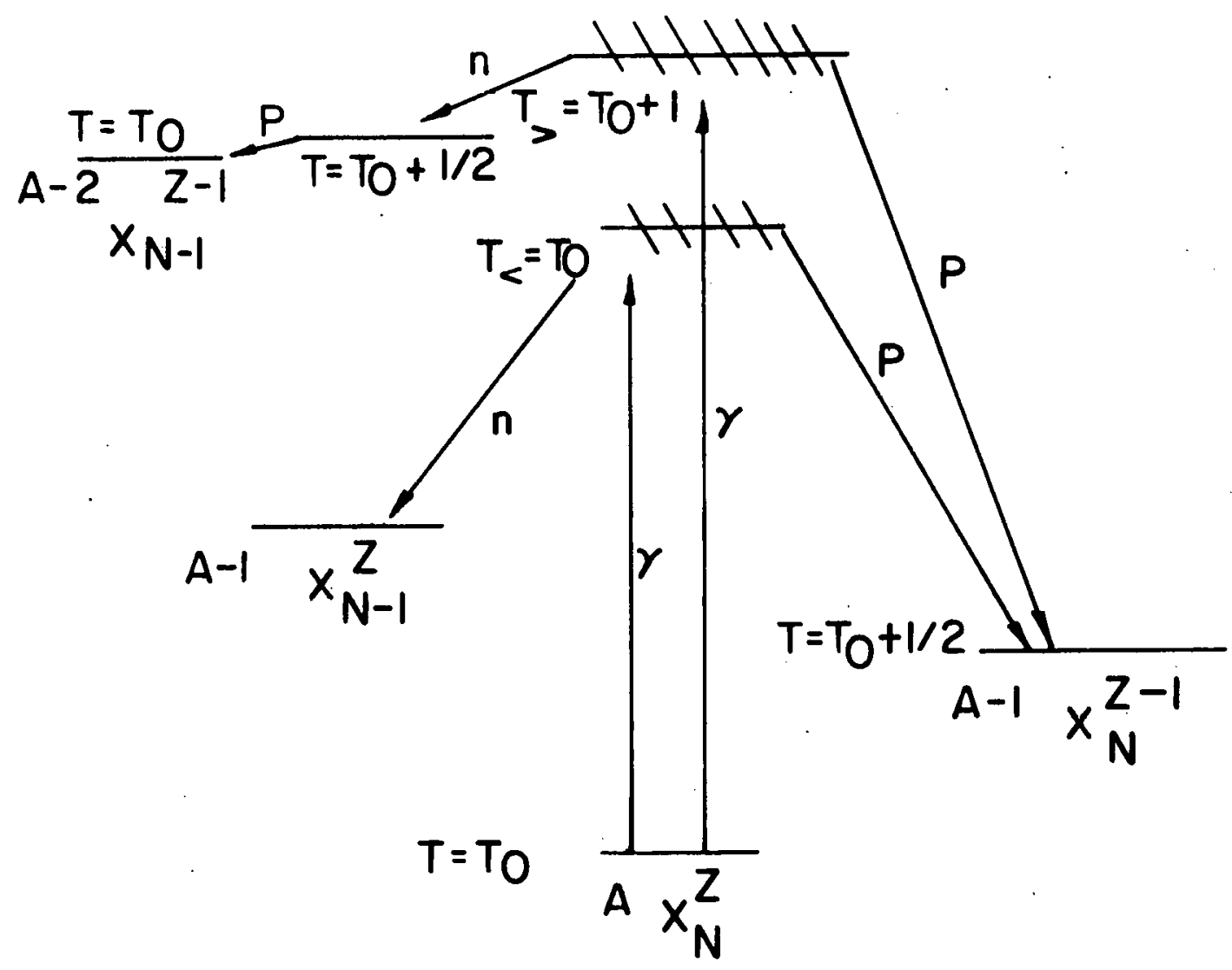

Figure 1. Isospin allowed transitions in the decay of the giant dipole resonance 
27

for an allowed decay is the isobaric analog to the ${ }^{A-1} \times{ }_{N}^{Z-1}$ ground state. A method to calculate the excitation energy of the analog states has been given by Jynecke (44). If this level is particle bound, $T_{\text {, }}$ strength should appear as a second peak in the $(\gamma, n)$ cross section. If the level is particle unstable, however, $T_{;}$strength should be seen in the $(\gamma, n p)$ partial cross sections. A careful study of these partial cross sections should reflect the operation of isospin selection rules. 


\section{CHAPTER III. EXPERIMENTAL INVESTIGATION}

\section{Activation Technique}

In this experiment, it is not practical to study all of the reaction channels by directly detecting the emitted particles. For example, in the $(\gamma, n p)$ reactions direct observation would require the measurement of neutrons and protons in coincidence. Since detection methods for these particles are drastically different the problem of measurement would be greatly complicated. These problems are conveniently avoided by measuring radioactivity generated in the target by the bremsstrahlung beam. In. past experiments at this laboratory, the annihilation radiation following $B^{+}$decay of the reaction products has been used to measure the induced radioactivity. These past measurements include the $(\gamma, n),(\gamma, n p)$ and $(\gamma, 2 n)$ reactions $i n z i n c$, and $(\gamma, n)$ reactions in $n i t r o g e n$, carbon and calcium. In this experiment only the $(\gamma, n),(\gamma, 2 n)$ and $(\gamma, n p)$ reactions in ${ }^{70} \mathrm{Ge}$ produce residual nuclei that are positron emitters; the $(\gamma, n p)$ reaction in ${ }^{72} \mathrm{Ge},{ }^{74} \mathrm{Ge}$ and ${ }^{76} \mathrm{Ge}$, the $(\gamma, n)$ reaction in ${ }^{76} \mathrm{Ge}$, and the $(\gamma, p)$ in ${ }^{76} \mathrm{Ge}$ and ${ }^{74} \mathrm{Ge}$ lead to residual nuclei that decay by emission of electrons, followed by emission of characteristic gamma rays. The other $(\gamma, n),(\gamma, p)$ and $(\gamma, 2 n)$ reactions lead to stable end products. Past procedures were not applicable in the case of these isotopes. However, since hlgh efficiency $\mathrm{Ge}(L I)$ detectors for gamma detection were aval lable in this laboratory, the residual radioactivity following the reaction of interest was measured by observing the proper gamma decays. Samples were exposed to bremsstrahlung radiation produced by the ISU 70 MeV Electron Synchrotron. The activated samples were counted by a gamma ray 
spectrometer, and the recorded spectra were analyzed to produce reaction yleld curves. This chapter describes the equipment used in the experimental investigation, the checks made to insure the accuracy of the apparatus, and the actual procedure followed to collect the data.

\section{Experimental Apparatus}

Samples

The germanium samples used in this experiment were discs of natural germanium of average welght 16 grams with average diameter $2.9 \mathrm{~cm}$ and thickness $0.5 \mathrm{~cm}$. Since germanium is very brittle, the samples had to be cast from $99.99 \%$ natural germanium and cut into slices with a spark cutter. The casting was done in a graphite crucible in an inert gas atmosphere. After cutting, the discs were dipped in an acid bath to remove oll and surface dirt. Samples varied in diameter by small amounts due to the casting procedure. Thirty-six samples were used to allow enough time to permit long-lived activities to decay away between successive activations.

Irradlation configuration and beam monltor

The source of photons used in this experiment was the isU $70 \mathrm{MeV}$ Electron Synchrotron. The geometry of sample activation is shown in Figure 2 .

When the electrons reached the desired energy, they were made to collide with a thin tungsten target. The resulting bremsstrahlung radiation passed through the wall of the vacuum chamber, through the beam collimator, and struck the germanlum sample. Most of the beam passed 


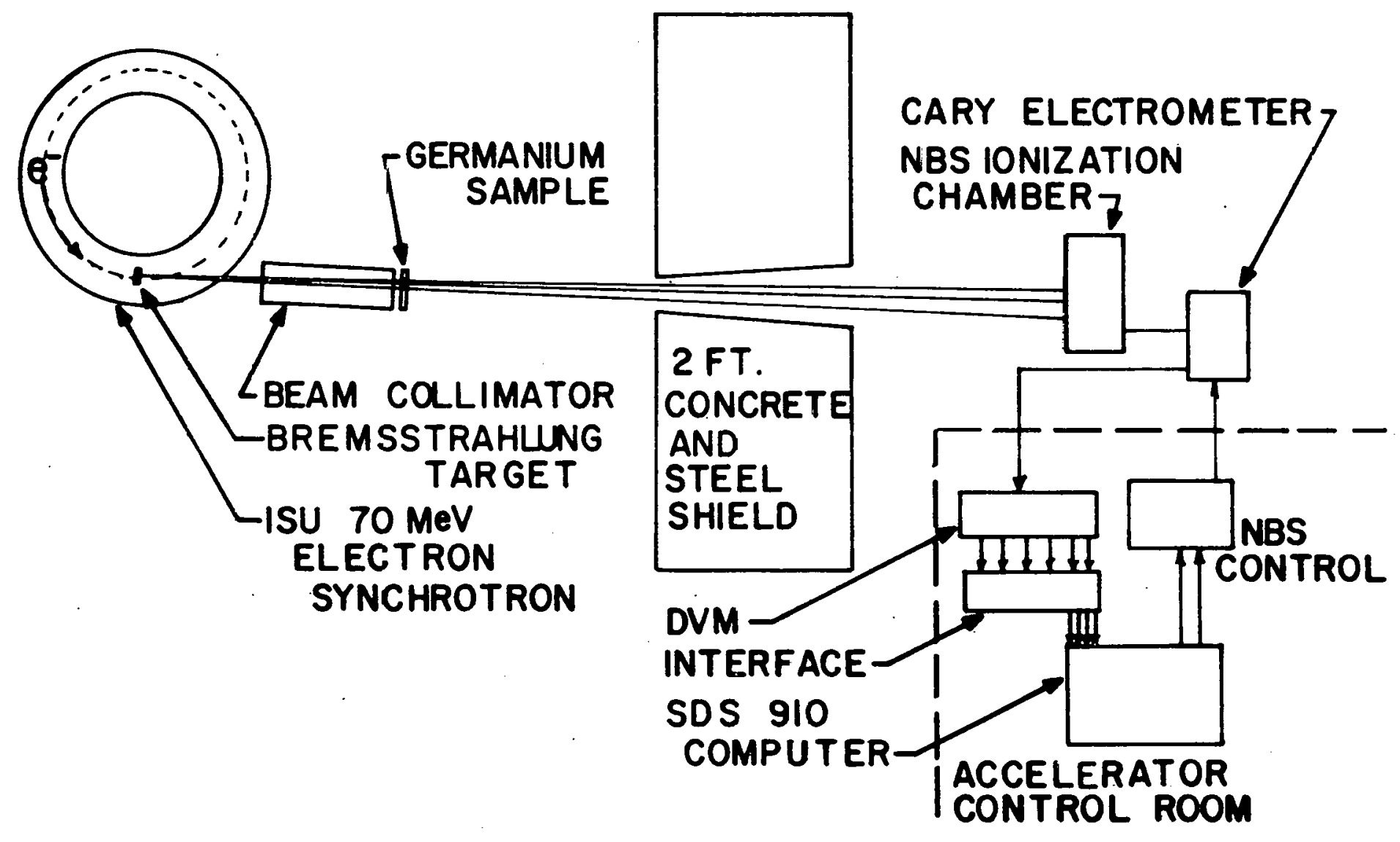

Figure 2. Beam geometry and dose monitor system 
through the sample and shielding wall and was intercepted by an NBS type P2 lonization chamber. The weak current produced in the chamber by the bremsstrahlung beam was integrated by charging a capacitor which was connected to a Cary Model 31 vibrating reed electrometer. The output voltage of the electrometer was proportional to the dose of radiation received by the sample. Output voltage of the electrometer was recorded by a Hughes Model 5000A digital voltmeter (DVM) interfaced to an SDS 910 computer. A discharge solenoid was also interfaced to the computer to discharge the integration capacitor on command.

The beam collimator was a cylinder of lead shot mixed with epoxy cement around a stainless steel tube. Inside this tube was a tapered lead insert, designed to prevent photon scattering from the walls of the collimator. The diameter of the collimated beam at the sample position was about $2.2 \mathrm{~cm}$ so that the beam was entirely eclipsed by the sample. After passing through a hole in the shielding wall the beam struck the Ionization chamber. This shleld wall protected the chamber from scattered photons and machine produced background. At the chamber, the beam de--.scribeld a circle about $13 \mathrm{~cm}$ in diameter.

Detectors and electronics

The gamma spectra of the activated samples were measured in two different photon spectrometers which will be referred to here as "counting houses." As originally designed both the counting houses had identical geometries, but fallure of a $\mathrm{Ge}(\mathrm{LI})$ detector during preliminary runs required a modlfied geometry. Figure 3 shows the actual counting house geometries and associated electronics. 


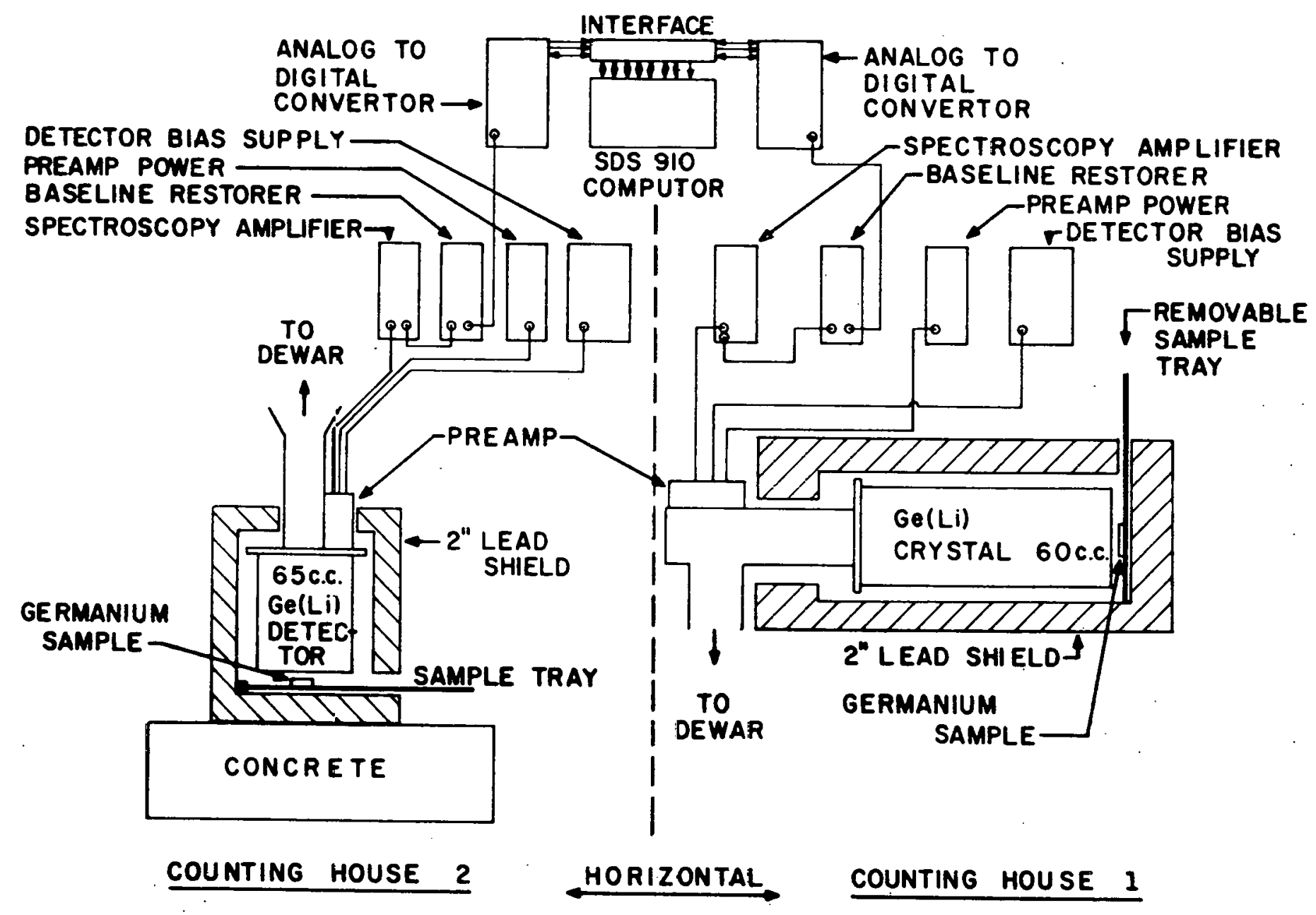

Figure 3. Counting house geometry and associated electronics 
Detectors Counting house 1 used a 60 c.C. Ge(LI) detector made by Ortec, Inc.; house 2 used a 65 c.c. detector made by Nuclear Diodes. Both detectors were blased by battery supplies; house 1 with +2550 volts, house 2 with +3060 volts. For these values of the bias, resolution on both detectors was better than $3.5 \mathrm{KeV} \mathrm{VWHM} \mathrm{on}{ }^{60} \mathrm{Co}$ lines. Both crystals were cooled to liquid nitrogen temperature and operated at that temperature.

Counting house geometry Activated samples were placed in front of the crystal $\mathrm{Ge}(\mathrm{LI})$ detectors on removable sample trays. The samples were held securely on the tray by a spring loaded clip, and the trays were aligned to position the sample at the center of the detector face. The detectors were mounted so that there was about $1 / 4$ inch between the sample and the detector housing. Past experlence showed that reproducible positioning of the samples is not a problem if reasonable care is taken by the experimenters. Since the detector diameter was considerably larger than the sample diameter, the solid angle for detection was nearly $2 \pi$.

Electronics Detector output pulses were amplified and shaped in a two step process. House 1 used an Ortec model 120-28 preamplifier followed by a Canberra Model 1417 Spectroscopy amplifier and Ortec Model 438 baseline restorer. House 2 used a Nuclear Diodes Model 101. preamplifier, Ortec filtered Spectroscopy amplifier Model $440 \mathrm{~A}$, and another Ortec Model 438 baseline restorer. Analog pulses from the two baseline restorers were d.c. coupled to two Victoreen Scipp Series analog to digital converters (ADCs). The ADCs were interfaced to an SDS 910 
computer. The pulses from baseline restorers (in the range 0 to 10 volts) were digitized into a 512 channel energy spectrum by the ADCs. At the end of the $A D C$ conversion cycle, the computer was interrupted and a location in core storage corresponding to the ADC address was incremented. During each counting period a gamma spectrum for the activated sample was collected in the computer memory.

On-line computer

The SDS 910 is a general purpose digital computer and was used to control data acquisition and perform some data reduction. The 910 has a 24 bit word length, 8196 words of magnetic core memory, and is equipped with a 16 channel priority interrupt system which allows on-line control of data acquisition. Extensive interfacing to external equipment allows the 910 to strobe, test, and accept data from many devices, including the ADCs mentioned in the discription of the counting system. The 910 is also capable of the output of analog signals for display and plotting. This feature was used to display real time status reports to a TV monitor in the accelerator control room. Typed output from the computer served as a monitor of data accumulation, and data was punched on paper tape for further processing.

\section{Calibrations and Accuracy}

The overall reproducibillty of a yield measurement is characterized by both the precision and the accuracy of the measurement. The precision of a measurement is an indication of the reproducibility of the measurement, the difference expected in the results of repeated measurements due 
to random error. The accuracy of an experiment denotes the difference due to systematic error between the results of measurement and the "true". value. A number of precautions were taken in this experiment to insure that yield measurements taken.with the equipment described in the last section would be both accurate and precise. These tests and calibrations will be briefly described in this section.

Beam alignment

Distortion of the bremsstrahlung beam due to scattering or shifts in beam position would directly effect the cross section measurement. Great care was taken to align the beam and prevent it from shifting position as the energy of the accelerator was changed.

The axis of the collimator was aligned with the beam axis using $x$-ray photographs. This alignment was checked by irradiating copper discs arranged in a cross pattern attached to the collimator. A plot of copper activity versus distance provided an accurage position of the beam axis. The beam axis was carefully located at energies of 15, 20, 30 and $40 \mathrm{MeV}$, and accelerator parameters adjusted until the beam remained centered to within $0.8 \mathrm{~mm}$ at the sample position for all energies.

In a similar manner the hole in the shielding wall and the ionization chamber were also centered on the beam axis. The hole in the wall was made larger than the beam diameter to prevent scattering distortions, and the beam was entlrely ecllpsed by the active part of the lonization chamber.

With constant beam geometry, the solid angles intercepted at the bremstrahlung target by the sample and ionization chamber were also 
constant. This is important for accurate yield measurements at all energles.

Energy control

Precise measurement of the experimental yield curve requires repeated runs at the same energy. It is essential that the energy control of the accelerator be very precise. The energy control system for the ISU Synchrotron has been discussed in detail (45) and experience has indicated that energies are reproducible to $\pm 10 \mathrm{KeV}$.

Immedlately prior to taking data, the absolute energy calibration of the synchrotron was checked by measurement of the sharp break in the $0^{16}(\gamma, n) 0^{15}$ yield curve at $17.27 \mathrm{MeV}$, and the break in the $\mathrm{Cu}^{63}(\gamma, n) \mathrm{Cu}^{62}$ at $10.8 \mathrm{MeV}$. The estlmated error on the $17.28 \mathrm{MeV}$ break is $36 \mathrm{KeV}$. The copper results was consistent with that error. Since germanium yield data were taken in $1 \mathrm{MeV}$ increments of the bremsstrahlung end point energy in this experiment, this uncertainty in the calibration is not serious.

\section{Dosemonitor accuracy}

Ionlzation chamber calibration The ionization chamber used in this experiment is a replica of the type P2 chamber which was developed and calibrated at the National Bureau of Standards by J. S. Pruitt and S. R. Domen (46). A calibration curve has been measured at the Bureau. In the following discussion the term "dose" is used to refer to the voltage measured by the digital voltmeter. The absolute calibration of the monitor will be discussed in the next chapter.

Accuracy checks Relative errors in the measured response of the ionization chamber, the "dose", will have a direct effect upon the 
measurement of ylelds. To determine the magnitude of these errors, frequent checks upon dosemonltor accuracy were performed during the experlment. The measurements were made by inserting a standard source of strontium 90 into a bracket on the NBS chamber. Electrons from this source pass through a thin window into the chamber and simulate ionization from the bremsstrahlung beam. The measurement consisted of recordIng the response of the system to this source for a large number of 10 second time intervals. The results of these checks showed a slight nonlinearlty in the response of the system for voltages above $5 / 6$ of a full scale reading of the electrometer. This nonlinear effect was in the order of $1 \%$ or less. The effect was eliminated by discharging the integrating capacitor (zeroing the dose reading) whenever the accumulated dose reached $2 / 3$ of full scale reading. A plot of dosemonitor checks indicated a small, slow drift in the system with time, but this was consistent with the standard deviation of the measurement. Daily measurement of the charge leakage of the system indicated a small, constant leakage of $0.06 \mathrm{volt} / \mathrm{hr}$. This corresponds to a dose measurement error of less than $0.5 \%$ for the lowest beam intensity.

Sample normalization

As noted earlier, the samples varied in diameter as a result of the preparation procedure. To prevent these factors from causing sample dependent yleld measurement fluctuations, a set of bombardments was made to obtaln sample normalization factors. These factors were applied to experimental yields to correct for sample difference: The correction factors calculated from these runs were all close to unity. The extreme 
values were 0.899 and 1.016 . The correctlon factors were determined to better than $1 \%$.

Another precaution was taken to ellminate the effect of variable sample geometry. Each sample was marked with an orlentation dot on its perimeter. Although the samples were measured to be flat within 0.001 Inch, the dots were always aligned with corresponding dots on the beam sample holder and counting house trays to ensure consistent sample geomet ry.

Spectrometer calibration and stability

Energy calibration A study of the decay schemes of the reaction end products revealed that all reactions could be observed by monitoring only gamma lines with energies between $100 \mathrm{KeV}$ and $1200 \mathrm{KeV}$. Using sources with lines of known energy, the gain of the system was adjusted until only this energy range was observed. The ADCs were callibrated with sources of ${ }^{22} \mathrm{Na},{ }^{137} \mathrm{Cs},{ }^{60} \mathrm{Co}$, and ${ }^{228} \mathrm{Th}$. The channel locations of known lines from these sources fixed the relation between channel number and gamma energy. This relation was found to be linear to within the accuracy of the measurement, better than $0.5 \%$.

Counting house normalization Since the two counting houses differed significantly in geometry and also since Ge(Li) crystals vary widely in their propertles, a procedure was devised to normalize the two houses. A serles of $40 \mathrm{MeV}$ runs with the same sample in both houses was performed before and during the experiment. The ratios of the yields from the two houses were used to normalize house 1 to house 2 . This procedure was followed for each of the gamma activities corresponding to 
the end products from each reaction studied. These norms were determined to better than $2 \%$ for four reactions and to no worse than $6 \%$ in the remaining ones. The normalization factors ranged in value from maximum of 3.40 to a minimum of 1.84 . This range of values was dependent upon gamma ray energy and reflects the energy dependence of the relative efficiency of the two Ge(LI) crystals. Differences in counting house geometry and absolute efficiency of the detectors also contributed to the size of the normalization factors.

Detector characteristics Both detectors possessed better than $4 \mathrm{KeV}$ FWHM resolution at the energy of the ${ }^{60}$ Co $1173 \mathrm{KeV}$ line. This resolution was more than adequate to separate the lines of each reaction end product. The relative efficiencies of both detectors were fitted to 4 th order polynomials in a least squares sense. The calculated efficiency curves were used to provide corrections to count rates for each gamma activity. The absolute efficiency of each detector was measured using a calibrated ${ }^{60}$ Co source. The values obtalined were $0.760 \% \pm$ $0.006 \%$ for house 1 and $1.086 \% \pm 0.012 \%$ for house 2 .

Count rate dependence The dependence of the spectrometer system upon counting rate can be critical in an activation experiment. This is true since counting rates will increase by orders of magnitude as the bombardment energy is increased. A count rate dependence would distort the measured yleld curve. In this experiment there are two possible sources of such rate dependence; gain shift and deadtime. In practice, neither effect was very great. A gain shift in the equipment would produce a shift in the FWHM of the gamma peak in the collected spectrum. 
In. the analysis of the spectra any shifts in peak centroid or FWHM were corrected for, however the observed shift was always less than $0.5 \%$. The deadtime for the system was measured using calibrated sources and found to be determined by the $A D C$. A precision pulser was used to measure $A D C$ deadtime as a function of frequency and amplitude. The results agreed closely with the manufacturer's specifications. For the maximum count rate of about 400 counts/sec., the observed deadtime was about $0.13 \%$. A correction for deadtime was included in the final analysis.

\section{Data Collection}

Yields for all 7 reactions of interest were measured in 1 MeV steps from $9 \mathrm{MeV}$ to $40 \mathrm{MeV}$. This range was chosen to start at an energy just below the lowest reaction threshold and extended beyond the resonances in the $(\gamma, n p)$ channel. For each end-point energy in this range at least 4 measurements of the yleld were made.

In a typical experimental yield run, the following sequence of procedures was employed: The sample to be activated was precounted for a five minute period to determine residual activity from previous activations and natural background; the sample was placed in the beam and irradiated for 34 minutes; after bombardment the sample was removed from the accelerator room to the counting house during a 1 minute waiting period; finally, the sample was counted for 40 minutes. The rate of data collection was accelerated somewhat by overlapping successive samples in this procedure; that is, as one sample was being I rradiated another could be in a precount or count period. After each two samples, collected spectra and other essential run data were output by the computer. 
The length of the precount-bombard-count cycle was determined after considerable thought, and experimental work. Since it was known how much time could be allotted to the experiment as a whole, the problem was to maximize the rate of data collection from seven different reactions In that perlod. The maximum rate for one reaction is related to the halflife of decay of its end product. In this experiment the half-lives ranged from elght minutes to nearly 40 hours.

Mathematically speaking, the simultaneous maximization of all rates is quite complex. Therefore, an experimental solution was used. A series of precount-bombard-count cycles of different length were performed, some. with different ratios of bombard-to-count times. The data collected were analyzed and errors in the yields were plotted versus cycle length. Since the total time length of the experiment was known, one could also compute the number of complete yield curves for each cycle length, and the statistical errors in the yields after $n$ curves. These curves of statistical error as a function of number of yield curves collected allowed selection of the appropriate cycle length that would minimize yield error at the end of the entire experiment for each reaction. The final choice of cycle length was chosen to most closely minimize yield error for all seven reactlons. This final value of cycle length corresponded to collection of 10 complete yield curves in 24 days. In the actual course of the experiment 6 complete and 2 partial curves were collected in 24 days. Delays due to equipment fallure caused the loss of the other data. The total experiment including normalization runs and calibrations was completed over a period of five and one-half weeks. 
In order to assure careful control of experimental timing and other aspects of data accumulation a computer program called Factory $V$ was written for the SDS 910 . The program consisted of several subroutines called by operator initiated interrupts. In addition to control of the yield measurements above, the computer controlled and recorded data for standard runs that monitored drifts in the dose and counting systems. Aside from the dose monitor checks with the strontium 90 source in the Ionization chamber, the yield was measured at $40 \mathrm{MeV}$ at least twice a day. These runs were identical to the yield measurement described above except that they were always run at the same energy. These runs served as a monitor of time dependent drifts in the whole system.

During any phase of the data collection, Factory $V$ gave a visual status report of the progress of each counting house. Accelerator operators and experimenters had only to glance at a $t . v$. monitor to be alerted to the next sample change or activation period. This display served to greatly reduce human errors during the experiment. As a check of impending disasters, Factory $V$ was used for partial data reduction of data collected and outputed crude yields after each run series. These yields were plotted during the experiment to provide motivation for the group and as a rough check of the quallty of the data. 
CHAPTER IV. DATA ANALYSIS

The photonuclear reaction cross section cannot be directly measured in an experiment of this type. The bremsstrahlung cross section is continuous in nature, and therefore the radiation spectrum from the synchrotron is continuous from zero photon energy to the energy of the accelerated electrons. For this reason, the cross section is not directly observable but must be deduced from an integral yield curve.

The actual yield data collected during this experiment were accumulated on punched paper type records from the SDS 910 computer. These paper tape records were later transferred to magnetic tape and direct access disk flies for further processing. The bulk of data analysis was performed on the lowa State University Computation Center's IBM 360/65 computer. The first section of this chapter presents the relation of the measured yields to the actual reaction cross section and the remaining sections describe the procedures used for processing the raw data into final cross sections. This description will be centered upon explanations of three major computer programs which were used to perform the analysis.

\section{Relation of Cross Section to Yield}

The cross section for a photonuclear reaction is related to the measured yield by the equation

$$
\alpha(E)=n_{s} \int_{0}^{\infty} N(E, k) \alpha(k) d k=\frac{N(E)}{D(E)}
$$


where

$$
\begin{aligned}
& \alpha(E)=\begin{aligned}
\text { the number of reactions per unit monitor } \\
\text { response (the yleld), }
\end{aligned} \\
& \begin{aligned}
& N(E)= \text { the number of reactions at bremsstrahlung end } \\
& \text { point energy } E,
\end{aligned} \\
& \begin{aligned}
& D(E)= \text { the monitor response (the "dose"), } \\
& n_{S}= \text { the number of target nuclei per } \mathrm{cm}^{2} \text { in } \\
& \text { the beam, } \\
& N(E, k)= \text { the number of photons of energy } k \text { per unit } \\
& \text { range of } k \text { which enter the sample per unit } \\
& \text { monitor response, for electron energy } E,
\end{aligned}
\end{aligned}
$$

and

$$
\sigma(k) \text { - the photonuclear cross section. }
$$

The incident photon spectrum, $N(E, k)$ can be expressed as

$$
N(E, k)=\left[\frac{\phi(E, k)}{k}\right] \frac{f_{s}(k)}{F(E)} .
$$

The factor enclosed in brackets is a renormalized form of the bremsstrahlung cross section of Schiff (47). It is written in this manner to emphasize the dominant $1 / k$ dependence. In terms of the Schiff function $\sigma(E, K), \phi(E, K)$ is defined as:

$$
\phi(E, k)=\frac{1}{16} \frac{137}{2 z^{2}}\left(\frac{m c^{2}}{e^{2}}\right) k \sigma(E, k) .
$$

$f_{s}(k)$ is a collective transmission function for all material between the bremsstrahlung radiator and the sample. $F(E)$ is a function which 
normalizes $N(E, K)$ to unit monitor response. It is a common practice to define the reduced yield, $Y(E)$, by substituting these functions for $N(E, K)$ :

$$
\begin{aligned}
& \alpha(E)=n_{s} \int_{0}^{\infty} \frac{\phi(E, k)}{\dot{k}} \frac{f_{s}(k)}{F(E)} \sigma(k) d k \\
& Y(E)=F(E) \alpha(E)=\int_{0}^{\infty} \frac{\phi(E, k)}{k} n_{s} f_{s}(k) \sigma(k) d k, \\
& Y(E)=\int_{0}^{\infty} \frac{\phi(E, k)}{k} s(k) d k ;
\end{aligned}
$$

where

$$
s(k) \equiv n_{s} f_{s}(k) \sigma(k)
$$

is defined to be the reduced cross section.

To calculate $F(E)$, it is necessary to examine the photon spectrum incident on the beam monitor. Let $N_{m}(E, k)$ be this spectrum:

$$
N_{m}(E, k)=\frac{\phi(E, k)}{k} \frac{f_{m}(k)}{F(E)}
$$

Here, $f_{m}(k)$ is the transmission function for all materials in front of the monitor. The energy in the spectrum is:

$$
E_{m}(E)=\int_{0}^{\infty} N_{m}(E, k) k d k=\frac{1}{F(E)} \int_{0}^{\infty} \phi(E, k) f_{m}(k) d k .
$$

We define $R(E)$ as the response of the monitor to each MeV of energy 
collected:

$$
R(E)=\frac{1}{E_{m}(E)}
$$

Thus we now can define $F(E)$ as

$$
F(E)=R(E) \int_{0}^{\infty} \phi(E, k) f_{m}(k) d k .
$$

The raw yields were obtained from the collected data spectra, and reduced by multiplication by $F(E)$. Calculation of $F(E)$ was straightforward. The voltage (dose) on the integration capacitor of the monitor system gave the charge collected during bombardment, and the collected charge was related to the deposited energy by Pruitt and Domen's calibrations (46). An experimental approximation to $f_{m}(k)$, the monitor trans$\mathrm{mission}$ function was obtained by measuring the monitor response in bombardments with a sample in position and comparing this to the response without a sample in position. This result was corrected for transmission through the accelerator vacuum chamber wall and the air in front of the monitor.

With the reduced yield determined, the reduced yield equation was solved for $s(k)$, the reduced cross section. The relative cross sections. were calculated from:

$$
\sigma(k)=\frac{s(k)}{n_{s} f_{s}(k)}
$$

The number of nuclei per $\mathrm{cm}^{2}$ in the beam, $n_{s}$, was calculated from the density of the samples. The function $f_{s}(k)$, which accounts for beam 
scattering by materials ahead of the sample, was calculated from known absorption coefficients of the materials between the bremsstrahlung radlator and the sample.

\section{Identification of Residual. Activities}

The number of reactions at each bremsstrahlung endpoint energy, $N(E)$, was calculated from the residual activity produced in the reaction prociss. Since the residual activlty was recorded as a gamma decay spectrum, calculation of $N(E)$ for each reaction requlred the precise identification of the gamma rays in the spectrum which were the result of that reaction. This section describes the analysis performed to identify the residual activities. All lines in the spectrum were identifled by the energy and the half-life of the activity.

\section{Energy determination}

A serles of activated germanium samples were counted in the spectrometer system. The resulting spectrum was analyzed and the energy of each peak in the spectrum determined using the known energy callbration of the system. The residual activity of each reaction was identified by comparing the peak energies with the known decay schemes of the reaction end-products. The results of this procedure are shown in Table 5 .

Half-life measurement

Another serles of activations was performed to determine the halflives assoclated with the gamma lines that had been previously identified by energy. In this manner positive identification of gamma lines resulting from the decay of reaction end-products was assured. Different 
Table 5. Gamma ray energies and half-lives of reaction end products

\begin{tabular}{|c|c|c|c|c|c|}
\hline Reaction & End Product & $\begin{array}{l}\text { Gamma Ray } \\
\text { Adopted } \\
\text { Value } \\
\quad \text { (keV) }\end{array}$ & $\begin{array}{c}\text { Energies } \\
\text { Our } \\
\text { Value } \\
\text { (keV) }\end{array}$ & $\begin{array}{l}\text { Half-Life } \\
\text { Adopted } \\
\text { Value }\end{array}$ & $\begin{array}{l}\text { Vaiues } \\
\text { Our } \\
\text { Value }\end{array}$ \\
\hline \multirow[t]{2}{*}{${ }^{76} \mathrm{Ge}(\gamma, n)$} & ${ }^{75} \mathrm{Ge}$ & 0.199 & $0.195(5)$ & $83 \mathrm{~min}$. & $80.1 \pm 2.6 \mathrm{~min}$. \\
\hline & & 0.264 & $0.259(5)$ & & \\
\hline \multirow[t]{3}{*}{${ }^{70} \mathrm{Ge}(\gamma, n)$} & ${ }^{69} \mathrm{Ge}$ & 0.574 & $0.572(5)$ & & \\
\hline & & 0.872 & $0.873(7)$ & $39 \mathrm{hr}$. & $30 \pm 11.2 \mathrm{hr}$. \\
\hline & & 1.106 & $1.112(10)$ & & \\
\hline \multirow[t]{2}{*}{${ }^{74} \mathrm{Ge}(\gamma, p)$} & ${ }^{73} \mathrm{Ga}$ & 0.296 & $0.293(5)$ & $4.8 \mathrm{hr}$. & $4.86 \pm 0.3 \mathrm{hr}$ \\
\hline & & 0.325 & $0.321(5)$ & & \\
\hline${ }^{76} \mathrm{Ge}(\gamma, \mathrm{np})$ & ${ }^{74} \mathrm{Ga}$ & 0.596 & $0.593(5)$ & $7.8 \mathrm{~min}$. & $8.6 \pm 2 \mathrm{~min}$. \\
\hline${ }^{74} \mathrm{Ge}(\gamma, \mathrm{np})$ & ${ }^{72} \mathrm{Ga}$ & 0.8347 & 0.8352 (7) & $14.1 \mathrm{hr}$. & $13.8 \pm 1.1 \mathrm{hr}$ \\
\hline${ }^{72} \mathrm{Ge}(\gamma, \mathrm{np})$ & ${ }^{70} \mathrm{Ga}$ & 1.042 & $1.055(15)$ & $21.1 \mathrm{~min}$. & $21.1 \pm 3.8 \mathrm{~min}$ \\
\hline${ }^{70} \mathrm{Ge}(\gamma, \mathrm{np})$ & ${ }^{68}{ }_{\mathrm{Ga}}$ & 1.0776 & $1.081(15)$ & $68.2 \mathrm{~min}$. & $67.6 \pm 2.9 \mathrm{~min}$. \\
\hline
\end{tabular}


length bombardment intervals were used to produce adequate numbers of residual nuclei. Various counting sequences were employed to minimize statistical errors in the decay curve for each half-life. The resulting decay curves were fitted by least squares techniques to logrithmic lines. The half-lives were determined from these fits and are also listed in Table 5. For comparison the accepted values for the half-lives are also given.

\section{Reduction of Raw Data}

Since data were recorded as a series of spectra containing peaks from all activities of interest, a procedure was devised to locate. the desired peaks, and obtain the area in each peak. The area of a peak divided by the corrected dose was the experimental yield. A computer program called PFA was used to extract these yields from the recorded spectra.

Proper yield calculation required development of several procedures. First, a reliable method of determining the location of each gamma line in the spectrum was required. The energy of the line could then be calculated from the ADC calibration. Second, if a line was properly identified as a decay of a reaction end-product, the area under the spectrum peak was to be calculated accurately. of course, this calculation had to apply the proper correction for background and residual activities. The next few paragraphs describe how PFA performed each of these tasks.

\section{Peak locations}

The energy calibration of the $A D C$ provided a good first estimate of the peak lncation. However, slight gain shifts due to count rate did 
effect peak positions. In some cases the presence of nearby lines from other decays could cause confusion. To avoid a time wasting search of the entire spectrum, but to account for the difficulties just mentioned PFA adopted the following procedure. A window of variable width is chosen about the probable peak position. The data in this window are searched and all peaks in the window are tagged and their energy calculated. The peak finding procedure was developed by. M. A. Mariscotti in Reference (48). The peak closest to probable location was chosen for further analysis. As a monitor of this decision process all peak energies and locations were output along with the chosen ones. Once a peak was chosen, a new analysis window was'selected to eliminate other peaks from contributing to the area calculation. In practice, a lower limit was placed on the width of this window to assure reproducible area calculatlons.

\section{Area calculation}

The actual peak areas were calculated by summing counts in the analysis window after calculating and stripping off the background counts. This area was corrected for the residual actlvity in the sample from previous bombardments, if any. The background was calculated by performing a least squares fit to a background sample chosen from the spectrum surrounding the peak position. A reduced chi-squared, defined by

$$
x^{2}=\frac{\left(y_{i}{ }^{\prime}-y_{i}\right)^{2}}{y_{i}{ }^{2}}
$$


where

and

$$
\begin{aligned}
& y_{1}{ }^{\prime}=\text { calculated background point, } \\
& y_{1}=\text { background sample point, }
\end{aligned}
$$

was monitored for each $f / t$ to the background. Values of $x^{2}$ ranged from 1.2 to 0.8 , with a value of 1.0 considered the "best" fit. The residual activity correction was computed from the precount spectrum for the appropriate run. The area of each pre-count peak was calculated in the manner described above. This area was corrected to allow for decay up to and durling the counting perlod. The corrected residual counts were then subtracted from the calculated area for the new activation.

The corrected peak area is proportional to the number of reactions produced during activation. The experimental yields were therefore calculated by dividing the peak area by the corrected dose. PFA was capable of calculating corrected doses, however once the factory $V$ values were verifled to be correct they were used in the yield calculation.

Several corrections were applied to the experimental ylelds by PFA. A small correction for charge leakage in the dose monitor, and large correction for the relative efficiency were applied.'

The results of PFA were punched on cards to be used in the next step of analysis, the formation of the reduced yield curves.

'Both quantities were described in Chapter 111. 


\section{Calculation of Reduced Yield Curves}

The yield curves calculated by PFA required further analys is before the cross sections could be obtained. Data reduction continued using another computer routine, called APU. This routine was developed over a long period of time by the synchrotron group and has been extensively documented in previous work $(19,28)$. The general purpose of this routine was to convert the yields of PFA into reduced yields suitable for processing by the CLSR (cross section extraction) routine. The major functions performed by APU were to correct for energy dependent and other systematic errors, calculate reduced yields for each run, the average yield at each energy, the errors in the yields and the errors in the average ylelds.

The yields were corrected for systematlc dependence on several experimental quantitles. Dependence on sample, house, count rate, betatron dose, and energy were included. The corrections were applied as multiplicative factors in such a manner that the fractional error in the yield remained constant. In the previous chapter, the measurement of the sample, house, and count rate corrections was described.

The energy dependent correction applied was multiplication by the monitor response function $F(E)$. The measured monitor transmission curve $f_{m}(k)$, was multiplied by the area under the bremstrahlung curve. This area was calculated by integrating the Schiff function (47). The total product function,

$$
F(E)=R(E) \int_{0}^{\infty} \phi(E, k) f_{m}(k) d k
$$

was repeatedly smoothed to prevent introduction of spurious structure into 
the yields and therefore the cross sections.

The transition from betatron to synchrotron mode in the acceleration cycle was a source of a systematic error usually referred to as the "betatron dose." During the transition not all of the electron beam is captured into the synchrotron orbit. Electrons lost in this process strike the walls of the beam chamber, or the target, and produce bremsstrahlung with an end point energy of about $4 \mathrm{MeV}$. This bremsstrahlung is too low in energy to produce reactions in the sample but stlll is monltored by the Ionization chamber. This additional contribution to the dose was measured to be $4 \%$ at $10 \mathrm{MeV}$. The analytical dependence of betatron dose is known (19) and this function was used to correct the yields. Valid solutions of the reduced yield curves by the least structure technique require that the errors in the yields are estimated accurately (49). A careful error analysis is performed by APU to determine the yleld errors.

Since several yield measurements were performed at each energy, an average yield, standard deviation and root mean square error due to counting statistics can be calculated at each energy. Statistical weights were used by APU to calculate the average yields. In general, the standard deviations will be larger than the random counting error, due to the effect of experimental errors other than random errors. Also, since there are a limited number of measurements at each energy, the standard deviations are not well determined, and are poor estimates of the yield errors. Yield errors are calculated by assuming that the experimental contribution to the error is a constant fraction of the average yield. 
That is,

$$
e_{i}^{2}=\varepsilon \bar{\gamma}_{i}^{2}
$$

Where $e_{i}$ is the experimental error in the yield $\bar{Y}_{j}$ at the ith energy.

$\varepsilon$ is estimated by calculating a weighted average of the difference between the standard deviation and the counting errors,

$$
\varepsilon=\frac{\sum_{i}\left[\left(s_{i}{ }^{2}-r_{i}{ }^{2}\right) / \bar{y}_{i}{ }^{2}\right] w_{i}}{\sum_{i} w_{i}}
$$

where

$$
\begin{aligned}
& s_{1}=\text { standard deviation at ith energy } \\
& r_{i}=\text { rms counting error, } \\
& w_{i}=\text { welghting factor. }
\end{aligned}
$$

The error in the yield for the $j$ th run at energy $i$ is then given by:

$$
\left(\Delta Y_{i j}\right)^{2}=\varepsilon Y_{i j}^{2}+r_{i j}^{2}
$$

The error in the average yield at the ith energy is,

$$
\left(\Delta \bar{Y}_{i}\right)^{2}=\left(\varepsilon \bar{Y}_{i}^{2}+r_{i}^{2}\right) / n_{i}
$$

$n_{i}$ being the number of runs at the ith energy. The weighting factors, $w_{i}$, are taken to be

$$
w_{i}=\left[\bar{y}_{i} /\left(\frac{1}{n_{i}} \sum_{j} \Delta y_{i j}\right)\right]^{4} n_{i}\left(n_{i}-1\right) .
$$

Since $\Delta \bar{Y}_{1}$ is included in $w_{1}$, the equation for $\varepsilon$ must be solved in an iterative manner. Iterations are performed until a newly calculated $\varepsilon$ differs from the orevlous one by a negligible amount. 
As defined above, the square root of $\varepsilon$ can be interpreted as the average fractional experimental error. Once $\varepsilon$ has been evaluated the average fractlonal statistical error, and the average fractional total error can be computed.

When the valld yield errors are obtained, residual errors can be calculated from

$$
R_{i j}=\frac{Y_{i j}-\bar{Y}_{i}}{\Delta Y_{i j}}
$$

where $R_{i j}$ is the residual for the $j$ th yield at ith energy. The residuals are an important tool in control of the analysis. Plots of the residual versus time, and an error histogram indlcating the distribution of residuals, provided a means of rapid location of runs with large deviations. A majority of these runs could then be discarded due to experimental errors (equipment or operator failure, etc.). The total number of discarded runs was small compared to the total runs in each curve.

It is important to note that the largest contributions to the $\varepsilon$ came from the values for runs near reaction threshold. This is as expected since it is at these very small yield values that the PFA area calculation is expected to be least reliable.

\section{Extraction of Cross Sections}

To extract the reduced cross section $S(k)$, it is necessary to solve the' reduced yield equation: 


$$
\begin{aligned}
& Y(E)=\int_{0}^{\infty} N(E, k) S(k) d k \\
& N(E, k)=\frac{\phi(E, k)}{k}
\end{aligned}
$$

The solution is difficult since the effect of statistical errors in the $Y(E)$ cause the solutions to oscillate at high values of $k$. Various methods of solution have been employed in the past, such as the "photon difference method" (50) and the method of Penfold and Leiss (51). In this experiment cross sections solutions were obtained using the method of "Least Structure" developed by B. C. Cook (52). The Least Structure procedure calculates the smoothest numerical solution statistically consistent with the reduced yields $Y(E)$ and their estimated errors. In practice a computer program, CLSR, was used to analyze the yield curves.

In the theory of CLSR a variational problem is solved to give, in matrix form,

$$
Y=\left(N+\lambda(\tilde{N})^{-1} W^{-1} \tilde{S}\right) S
$$

where

$Y$ is a vector of yields,

$N$ is the bremsstrahlung matrix,

$\lambda$ is the varlational parameter,

$W$ is diagonal with elements $w_{i j}=\left(L / Y_{i}\right)^{2}$,

$\tilde{S}$ is the smoothing matrix, which plcks out squares of second differences in $S$,

$S$ is a vector of calculated cross section values.

We can write for simplicity

$$
Y=M S
$$


and the solution is

$$
S=M^{-1} Y
$$

since $M$ is a square matrix. In CLSR solutions are obtained for different values of $\lambda$ until a solution is found which minimizes the second difference function

$$
\tilde{s}_{1}=\sum_{i} 1 / 4\left(s_{1-1}-2 s_{1}+s_{1+1}\right)
$$

and which satisfies the chi-squared test,

$$
x^{2} \equiv \sum_{i} \frac{\left(\bar{Y}_{i}-Y_{i}\right)^{2}}{\left(\Delta Y_{i}\right)^{2}} \cong n
$$

where $n$ is the number of energy steps in the yield curve. The Least Structures technlque has been critically evaluated and compared to other methods by Bramanis et al. (49).

\section{: $b$}

Determination of Absolute Cross Sections

After the least Structures procedure produced the $S(k)$, a number of normalization factors were applied to the solutions to obtain cross sections in $\mathrm{mb}$ unlts. The final cross sections were calculated from:

$$
\sigma(k)=\frac{s(k)}{n_{s} C_{P} C_{N_{2}} C_{D} C_{\mid A B} C_{B R} \varepsilon}
$$

where

$n_{s}$ is the number of nuclei per $\mathrm{cm}^{2}$ in the beam,

$C_{p}$ is a correction for pressure in the lonization chamber,

$\mathrm{C}_{\mathrm{N}_{2}}$ is the correction for using $\mathrm{N}_{2}$ instead of air in the Ionization chamber, 
$C_{D}$ is the absolute normalization constant for the chamber response,

$C_{I A B}$ is the correction for isotopic abundance,

$C_{B R}$ is the correction for gamma ray branching,

$\varepsilon$ is the normalization constant for detector efficiency in.

$2 \pi$ counting mode.

$n_{s}$ was calculated from the sample density. The densities were obtained by averaging the densities calculated from the samples themselves.

Since our ionization chamber was sealed and filled with pressurized $\mathrm{N}_{2}$, this differed slightly from the calibration conditions of Pruitt and Domen. Using correction functions suggested in their report $\mathrm{C}_{\mathrm{p}}$ and $\mathrm{C}_{\mathrm{N}_{2}}$ were calculated. Both constants had a value close to one.

The monitor response in volts had to be converted to measured charge in order to apply the monitor calibration of Pruitt and Domen. The capacitance of the integration capacitor was measured and is the constant $C_{D}$.

The correction for gamma ray branching ratios, $C_{B R}$, is the least well known of all the correction factors. Decay schemes in Lederer et al. (53) Nuclear Data $(54 a, b)$, and more recent literature were studied to determine these correction factors. The values for each reaction, their error and reference are listed in Table 6 . The errors in these factors introduced the largest uncertainties into the final normalization.

$\varepsilon$, the absolute efficiency of the detectors was measured using a calibrated radiolsotope source of ${ }^{60} \mathrm{Co}$, and was determined to about $1 \%$ precision. 
Table 6. Gamma ray branching

\begin{tabular}{|c|c|c|c|c|}
\hline Reaction & $\begin{array}{l}\text { Gamma } \\
\text { Energy } \\
\text { (MeV) }\end{array}$ & $\begin{array}{l}\text { Relative } \\
\text { Branching }\end{array}$ & $\begin{array}{l}\text { Fraction of } \\
\text { Total Decays }\end{array}$ & References \\
\hline \multirow[t]{3}{*}{${ }^{70} \mathrm{Ge}(\gamma, n)$} & 0.573 & 0.47 & & \\
\hline & 0.872 & 0.38 & & \\
\hline & 1.107 & 1.00 & $0.281 \pm 0.03$ & $55,56,57$ \\
\hline \multirow[t]{2}{*}{${ }^{76} \mathrm{Ge}(\gamma, n)$} & 0.198 & 0.123 & & . \\
\hline & 0.2646 & 1.00 & $0.108 \pm 0.013$ & 58,59 \\
\hline \multirow[t]{2}{*}{${ }^{74} \mathrm{Ge}(\gamma, \mathrm{p})$} & 0.295 & 1.00 & $0.48 \pm 0.078$ & 60,61 \\
\hline & 0.325 & 0.13 & & \\
\hline${ }^{70} \mathrm{Ge}(\gamma, \mathrm{np})$ & 1.0776 & 1.00 & $0.042 \pm 0.006$ & 55,56 \\
\hline${ }^{72} \mathrm{Ge}(\gamma, \mathrm{np})$ & 1.040 & 0.05 & $0.005 \pm 0.0018$ & 62,63 \\
\hline${ }^{74} \mathrm{Ge}(r, n p)$ & 0.835 & 1.00 & $0.950 \pm 0.058$ & 64,65 \\
\hline${ }^{76} \mathrm{Ge}(\gamma, \mathrm{np})$ & 0.598 & 1.00 & $0.516 \pm 0.093$ & 66 \\
\hline
\end{tabular}


CHAPTER V. EXPERIMENTAL RESULTS

\section{Cross Section Results}

The photonuclear cross sections extracted from the yields measured in this experiment are presented in Figures 4 through 10 . The vertical error bars are an indication of the total error in the reduced cross section as calculated by the analysis. The horizontal error bars are not uncertainties in the energles. Rather, they are a measure of the degree of smoothing performed by the Least Structures analysis. If the physical cross section were shaped like a delta function resonance, the Least Structure analysis would produce a smoothed resonance with a width at half maximum equal to the horizontal error bar. Thus, while the peak positions and areas in the cross section are reasonably accurate, the widths are not nearly as quantitative.

The $(r, n)$ cross sections

The single neutron cross sections for ${ }^{70} \mathrm{Ge}$ and ${ }^{76} \mathrm{Ge}$, shown in Figures 4 and 5 , each display two broad maxima. The first resonance has the famlliar glant resonance shape peaking at about $16 \mathrm{MeV}$ with an average peak height of $125 \mathrm{mb}$. These results are consistent with the usual collective model treatment of the giant resonance.

The second resonance in the $(\gamma, n)$ channel is not so easily described. It appears at about $27 \mathrm{MeV}$ in ${ }^{76} \mathrm{Ge}$, and at about $34 \mathrm{MeV}$ in ${ }^{70} \mathrm{Ge}$. The integrated cross section in the second peak is about $1 / 4$ that of the first peak for ${ }^{70} \mathrm{Ge}$, and about $1 / 3$ of the first peak in ${ }^{76} \mathrm{Ge}$. The appearance of this sizable strength at energies this much above the giant resonance 
61

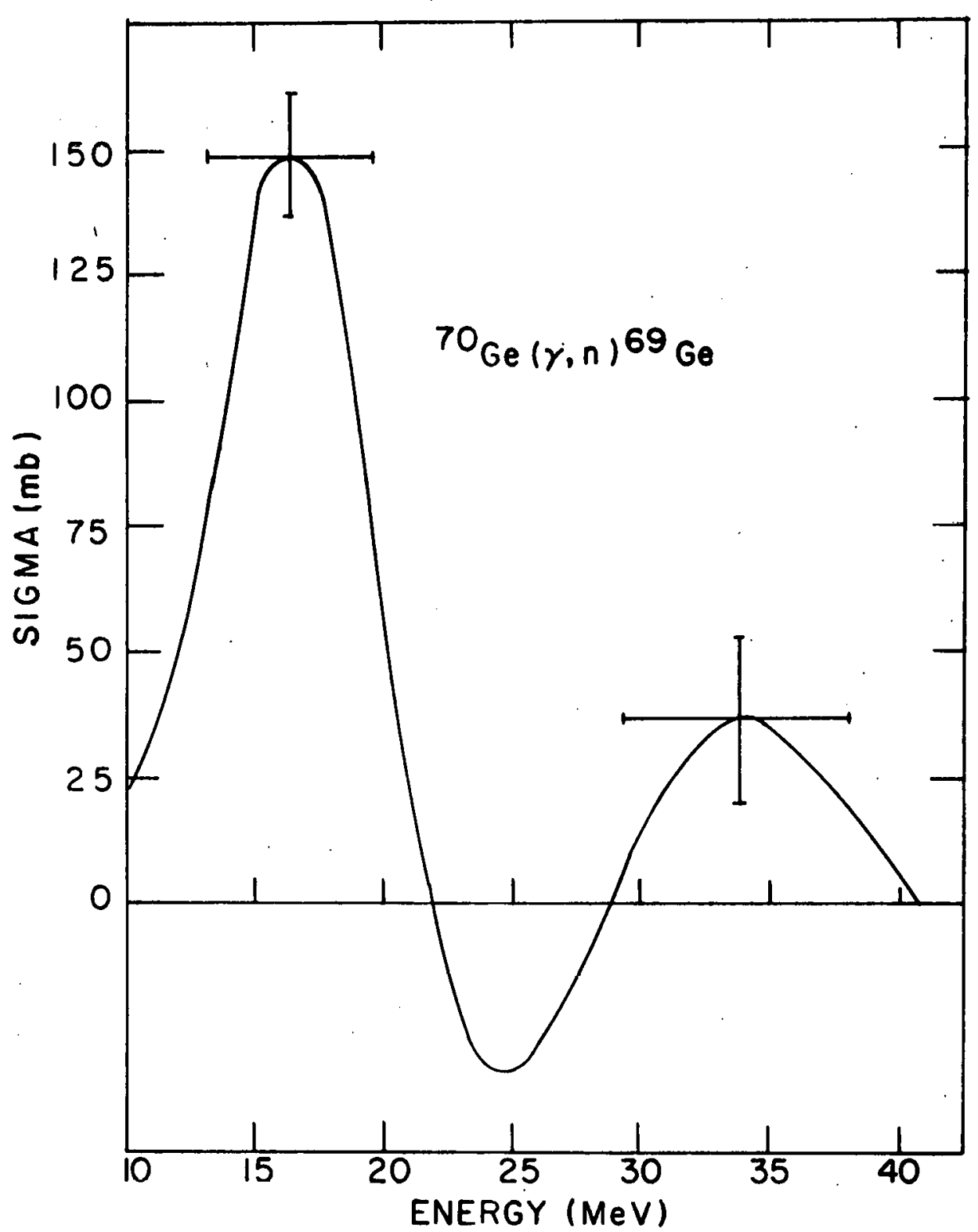

Figure 4. Cross section of ${ }^{70} \mathrm{Ge}(r, n){ }^{69} \mathrm{Ge}$ 


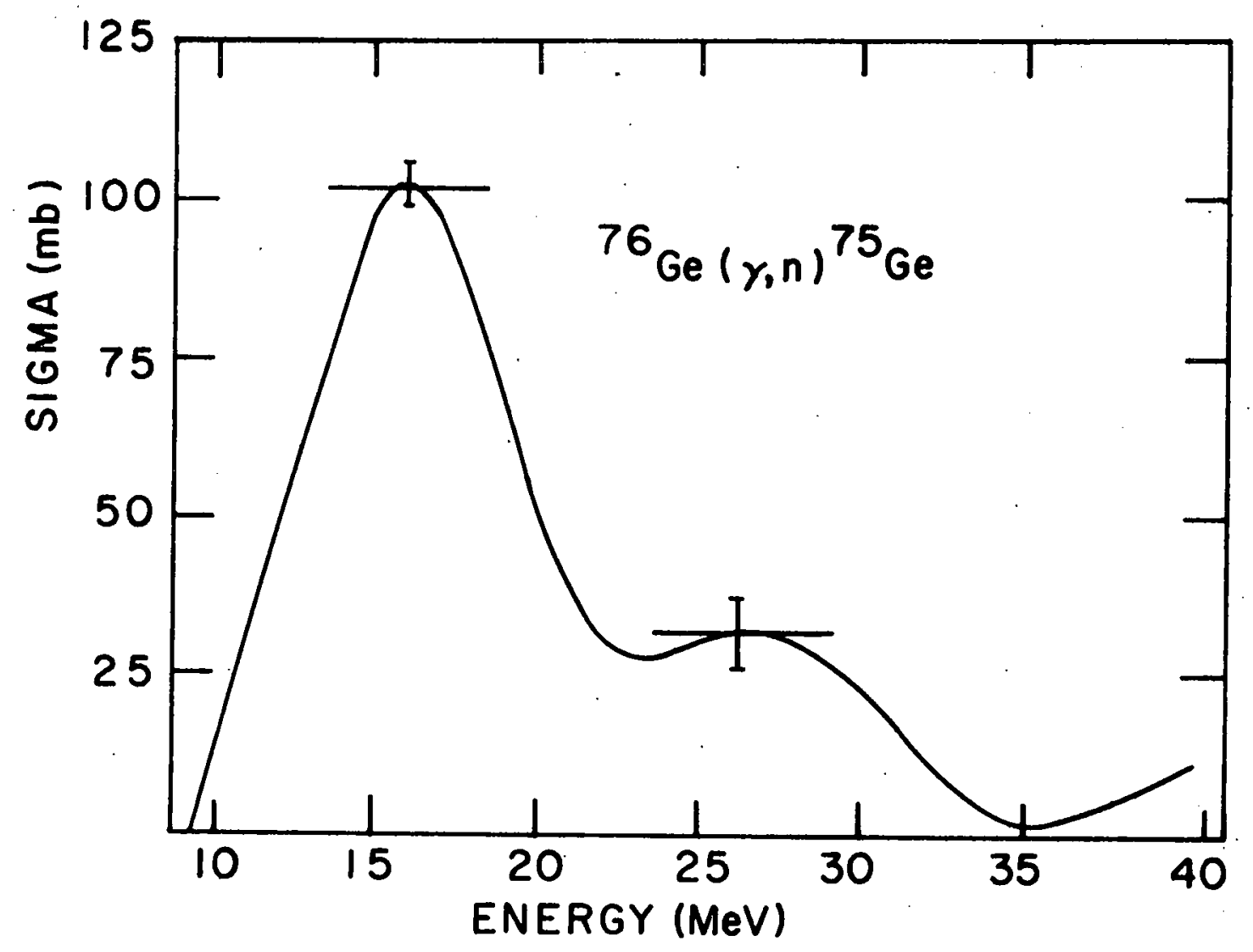

Figure 5. Cross section of ${ }^{76} \mathrm{Ge}(\gamma, n)^{75} \mathrm{Ge}$ 
energy is not predicted by simple collective or single particle models. The large negative dip in the ${ }^{70}$ Ge cross section is a result of analysis and will be discussed in the section of this chapter dealing with sources of error.

The ${ }^{74} \mathrm{Ge}(\gamma, p)$ cross section

The single proton cross section for ${ }^{74} \mathrm{Ge}$ is shown in Figure 6 . Structure in the cross section is observed at about 18,24 and $30 \mathrm{MeV}$. The cross section has its peak value of about $9 \mathrm{mb}$ at $24 \mathrm{MeV}$, and the major portion of the total integrated cross section is obtalned from the peaks at 24 and $30 \mathrm{MeV}$. The "talls" noted below $12 \mathrm{MeV}$ and above $36 \mathrm{MeV}$ are agaln artlfacts of the analysis and will be discussed later.

The $(y, n p)$ cross sections

Three of the four $(\gamma, n p)$ cross sections are measured unambiguously by this experiment; the result for ${ }^{74} \mathrm{Ge}(\gamma, \mathrm{np})$ is however contaminated by the ${ }^{73} \mathrm{Ge}(\gamma, \mathrm{p})$ reaction whlch populates the same residual nucleus. The resulting sum of the ${ }^{73} \mathrm{Ge}(\gamma, \mathrm{p})$ and the ${ }^{74} \mathrm{Ge}(\gamma, n p)$ cross section is shown in Figure 9. No definitive means of separating these results exists until the ${ }^{73} \mathrm{Ge}(\gamma, p)$ cross section can be independently measured by other methods. We can, however, interpret some of the features of the observed cross section. The strength at $17 \mathrm{MeV}$ is below the $(\gamma, n p)$ threshold by several $\mathrm{MeV}$, and can definitely be assigned to the ${ }^{73} \mathrm{Ge}(\gamma, \mathrm{p})$ reaction. Also since there is little reason to belleve that the ${ }^{73} \mathrm{Ge}(\gamma, p)$ cross section differs drastically from the ${ }^{74} \mathrm{Ge}(\gamma, p)$ cross section, most of the strength above about $34 \mathrm{MeV}$ can probably be assigned to the ${ }^{74} \mathrm{Ge}(\gamma, \mathrm{np})$ reaction. 


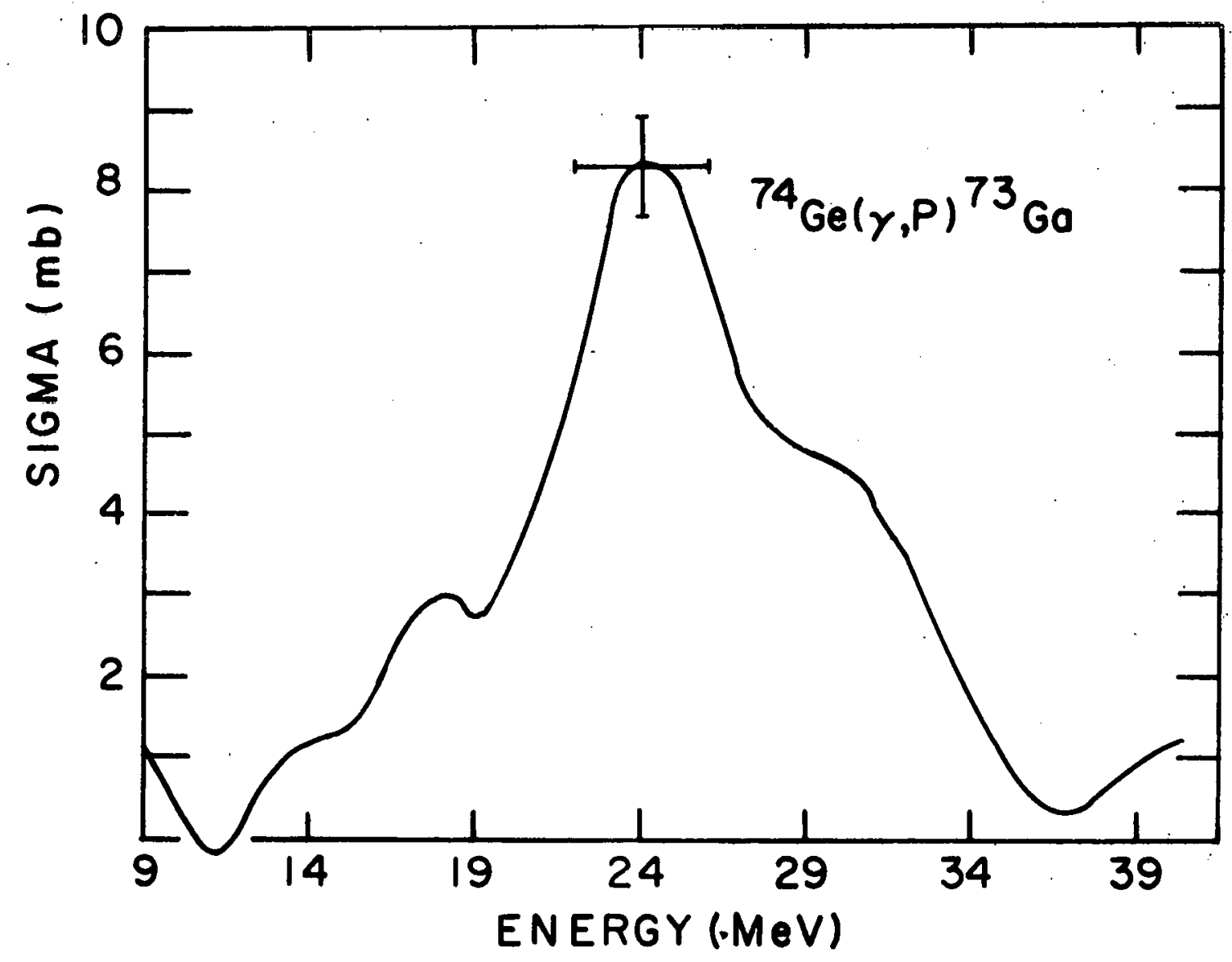

Figure 6. Cross section of ${ }^{74} \mathrm{Ge}(\gamma, \mathrm{p})^{73} \mathrm{Ga}$ 


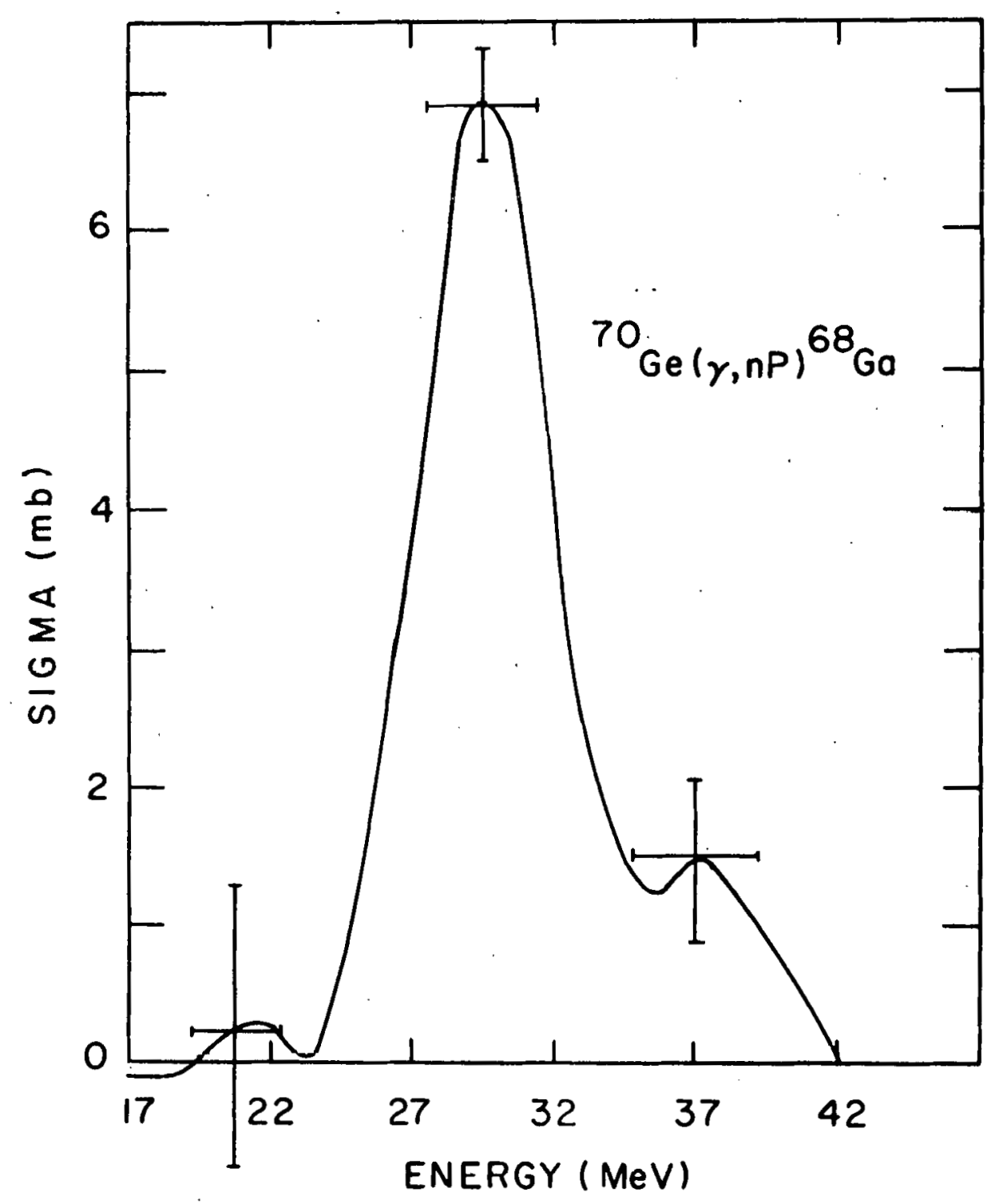

Figure 7. Cross section of ${ }^{70} \mathrm{Ge}(\gamma, \mathrm{np})^{68} \mathrm{Ga}$ 


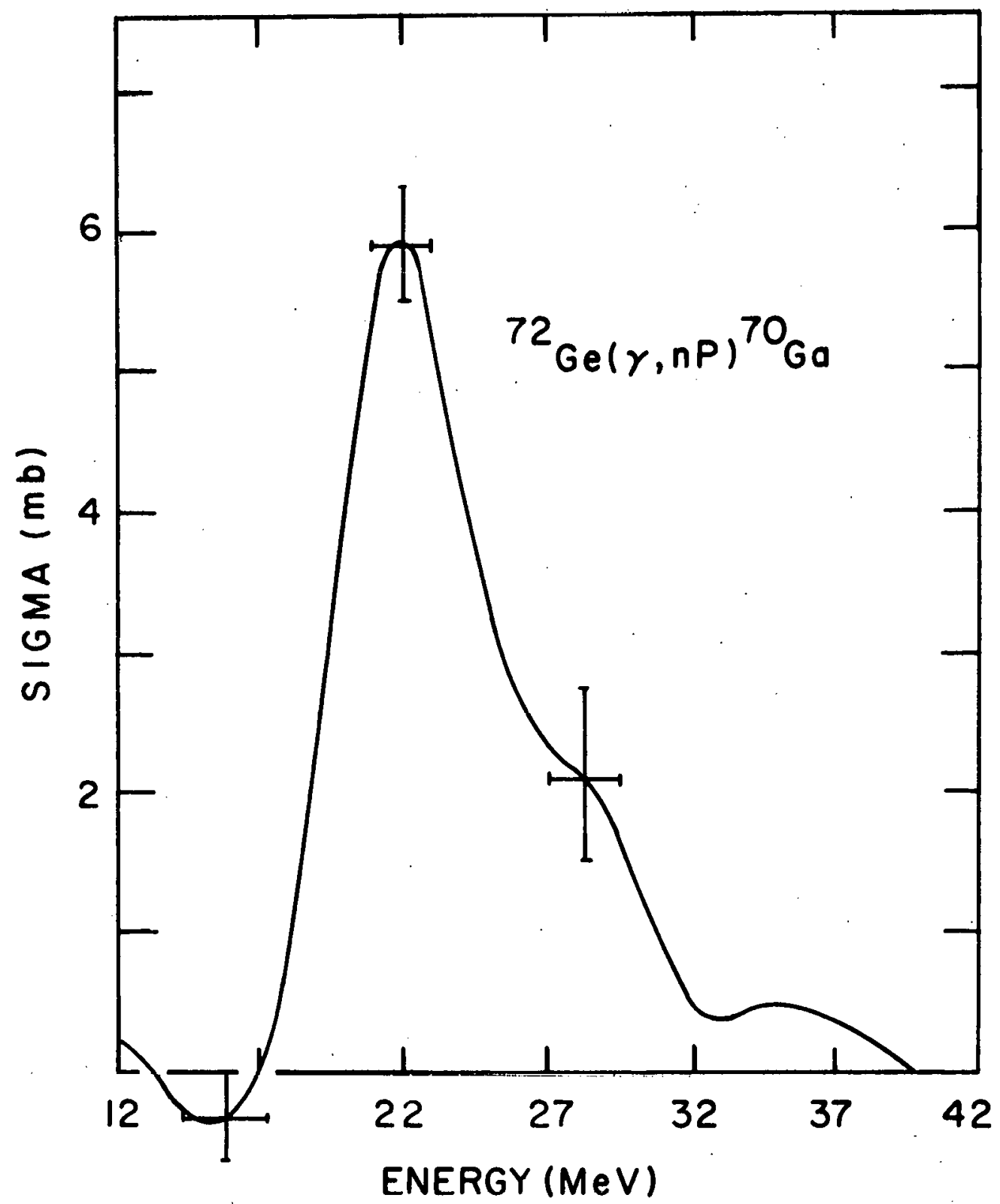

Figure 8. Cross section of ${ }^{72} \mathrm{Ge}(\gamma, \mathrm{np}){ }^{70} \mathrm{Ga}$ 
Figure 9. Cross section of $0.82{ }^{74} \mathrm{Ge}(\gamma, n p)^{72} \mathrm{Ga}+0.18^{73} \mathrm{Ge}(\gamma, p){ }^{72} \mathrm{Ga}$ 


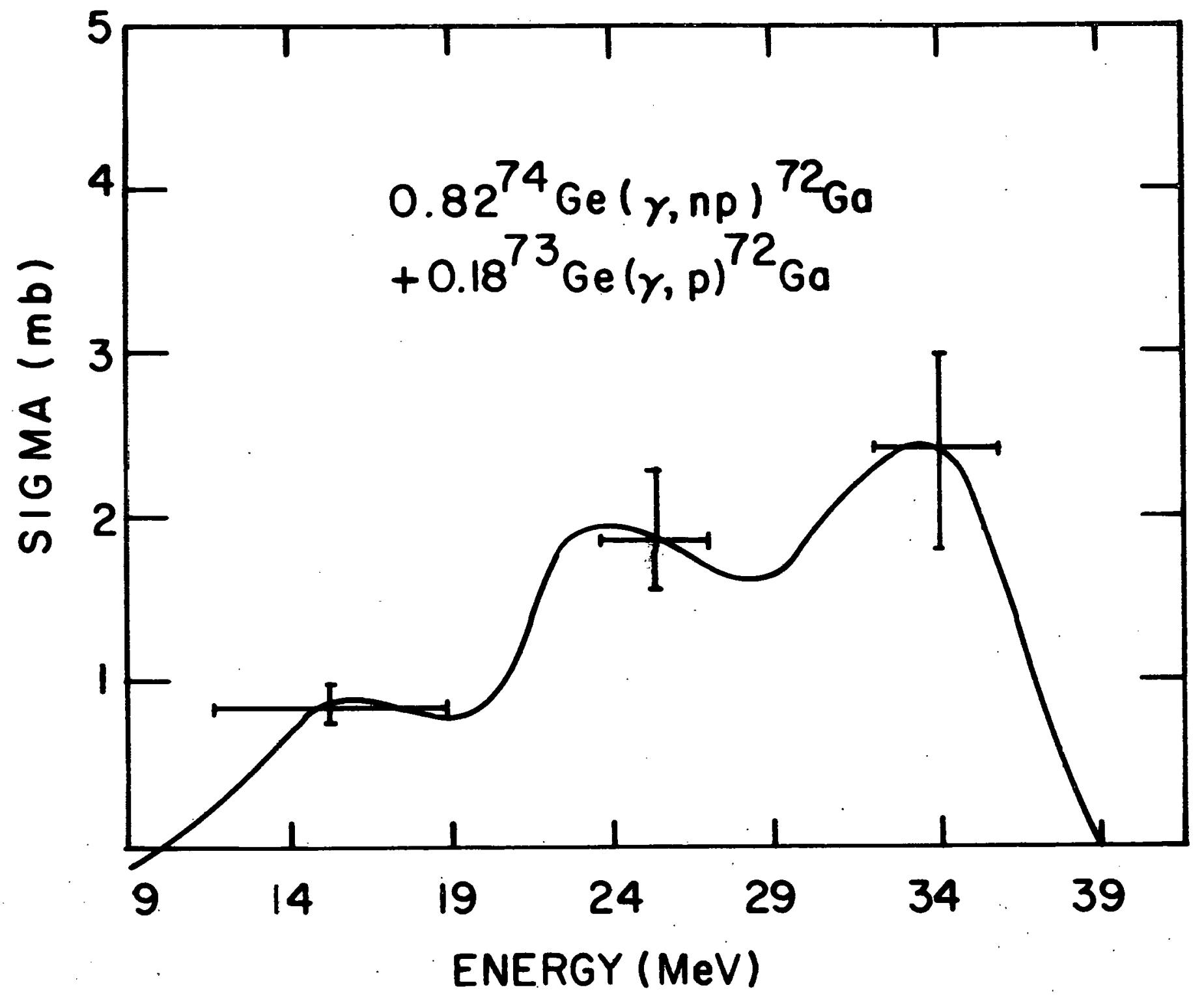

\&) 


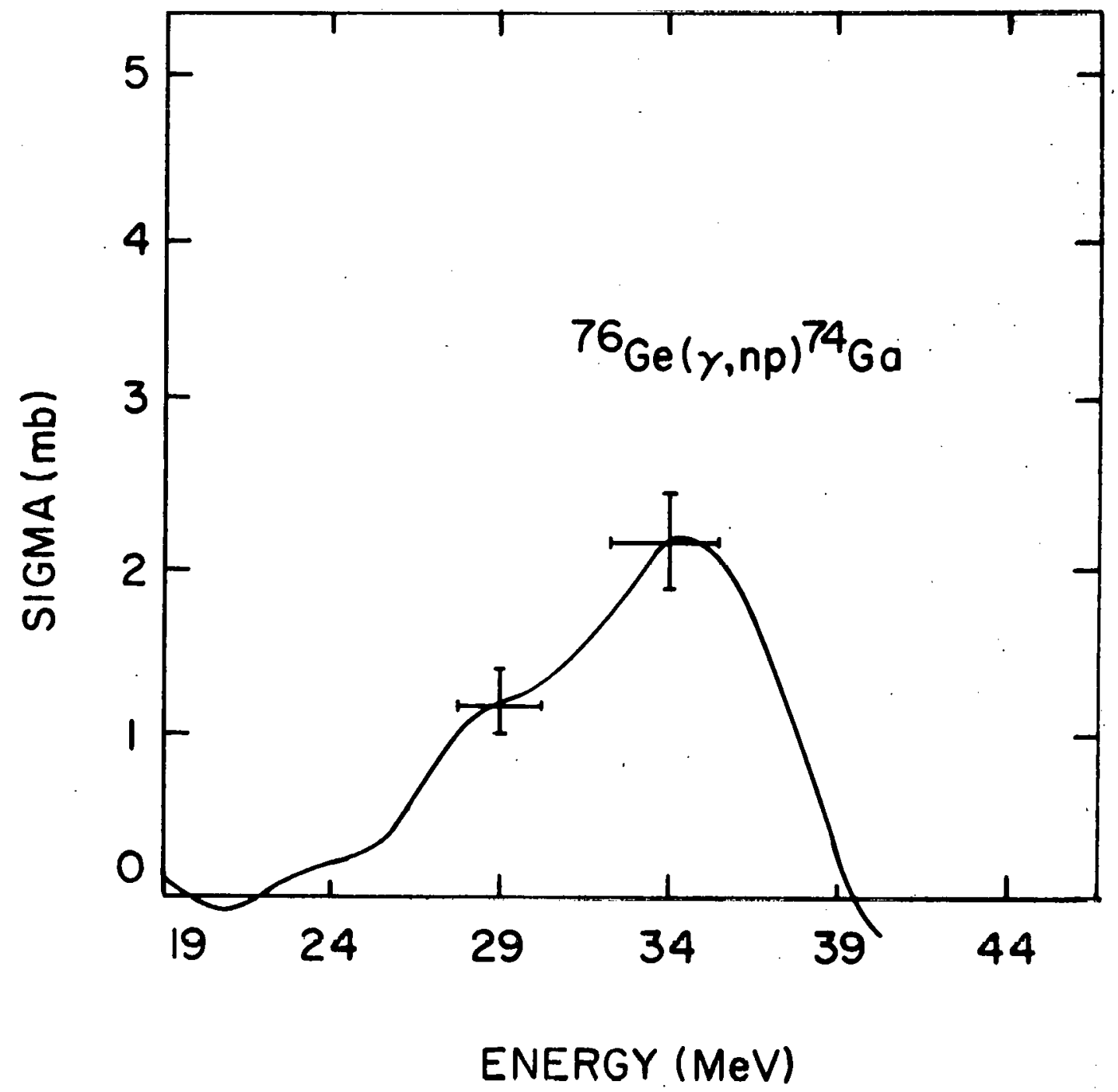

Figure 10. Cross section of ${ }^{76} \mathrm{Ge}(\gamma, \mathrm{np}){ }^{74} \mathrm{Ga}$ 
The remaining three $(\gamma, n p)$ cross sectlons, presented in Figures 7 , 8 and 10 possess some simllar features and one notable difference. The cross sectlons are similar in that all show resonance peaks at $29 \mathrm{MeV}$ and $36 \mathrm{MeV}$. The ratio of strength at $29 \mathrm{MeV}$ to that at $36 \mathrm{MeV}$ systematically decreases from a maximum value of 6 in ${ }^{70} \mathrm{Ge}$, to 5 in ${ }^{72} \mathrm{Ge}$, and finally to a value of $0.6 \ln { }^{76} \mathrm{Ge}$.

The ${ }^{70} \mathrm{Ge}(\gamma, \mathrm{np})$ cross section has a maximum of about $7 \mathrm{mb}$ at $29 \mathrm{MeV}$. ${ }^{72} \mathrm{Ge}(\gamma, \mathrm{np})$ has its maximum of about $6 \mathrm{mb}$ at $22 \mathrm{MeV}$, and ${ }^{76} \mathrm{Ge}(\gamma, \mathrm{np})$ cross section is peaked at $36 \mathrm{MeV}$ with a maximum value of $2 \mathrm{mb}$. The integrated cross section to $40 \mathrm{MeV}$ is greatest in ${ }^{70} \mathrm{Ge}(r, n p)$ and declines to about half the ${ }^{70} \mathrm{Ge}$ value for the ${ }^{76} \mathrm{Ge}(\gamma, \mathrm{np})$ cross section.

The notable difference in these cross sectlons is the appearance of the large peak at $22 \mathrm{MeV}$ in the ${ }^{72} \mathrm{Ge}(\gamma, \mathrm{np})$ cross sectlon. This resonance represents the largest fraction (67\%) of the total strength of this reaction.

Table 7 presents a summary of the main features of the cross section results. Further discussion and interpretation of these results can be found in Chapter 6.

\section{Sources of Error}

In addition to the systematic and random errors discussed in the prevlous chapter, there are several effects that could cause distortion of the final cross section results. These effects are for the most part characteristic of the method of analysis and as such are most difficult to adjust for.

Smoothing of the ylelds during Least Structures analysis is necessary. 
Table 7. Summary of cross sectlon results

\begin{tabular}{llcl}
\hline Reaction & $\begin{array}{l}\text { Position of } \\
\text { Maxima }(\mathrm{MeV})\end{array}$ & $\begin{array}{c}\text { Maximum } \\
\text { Vai ue }(\mathrm{mb})\end{array}$ & $\begin{array}{l}\text { Integrated Strength } \\
(\text { to } 40 \mathrm{MeV})(\mathrm{MeV}-\mathrm{mb})\end{array}$ \\
\hline${ }^{70} \mathrm{Ge}(\gamma, n)$ & 16,34 & 150 & $1086 \pm 119$ \\
${ }^{76} \mathrm{Ge}(\gamma, n)$ & 16,27 & 100 & $1077 \pm 129$ \\
${ }^{74} \mathrm{Ge}(\gamma, p)$ & $18,24,30$ & 8.9 & $92.0 \pm 10.0$ \\
${ }^{70} \mathrm{Ge}(\gamma, \mathrm{np})$ & 29,37 & 6.9 & $39.3 \pm 5.5$ \\
${ }^{72} \mathrm{Ge}(\gamma, n p)$ & $22,28,36$ & 5.8 & $38.9 \pm 14.0$ \\
${ }^{74} \mathrm{Ge}(\gamma, n p)$ & $16,25,35$ & 2.4 & $35.5 \pm 3.2$ \\
$76 \mathrm{Ge}(\gamma, n p)$ & 29,35 & 2.2 & $15.2 \pm 2.7$ \\
\end{tabular}

${ }^{a}$ These entries are calculated from the measured result which is $0.82^{74} \mathrm{Ge}(\gamma, \mathrm{np})^{72} \mathrm{Ga}+0.18^{73} \mathrm{Ge}(\gamma, \mathrm{p})^{72} \mathrm{Ga}$. 
because of random statistical errors in the measured ylelds. The Least Structures procedure produces a smooth cross section at the expense of resolution. As the random error increases, resolution decreases, and can cause non-physical cross section contributions. In this experiment random errors were particularly large in two instances.

First, for all cross sections, calculation of the yield is very poor near threshold because of large background-to-foreground ratios. Even though these points possessed correspondingly large uncertaintles, the unfolded cross sections reflect the distortion in the yields by slight shifts of qlant resonance toward a lower peak energy, and slight negative cross section preceding the first resonance.

The second contribution to increased random error. is the correction for activity in the sample remaining from a prevlous bombardment. This effect was the most prominent in the $40 \mathrm{hr}$. ${ }^{70} \mathrm{Ge}$ activity. In the worst case, slightly less than one half-life separated activations. In this case, a relatively large correction to the yield was necessary, with addition of a larger error. The error is most harmful in lower energy runs where the slope of the yield curve is changing rapldiy. This error is the maln reason for the large overshoot on the high energy side of the ${ }^{70} \mathrm{Ge}(\gamma, n)$ giant resonance.

The Least Structures procedure itself is a possible source of error. It is a characteristic of all analyses of photonuclear yield functions that "secondary resonances" may in reality be osclllations in the particular solution of the yleld equation. In a relatively low resolution experiment such as this one the Least Structure method is generally 
rellable if good error estimates are employed (49). Several tests of the particular solutions were made to assure that the observed secondary $(\gamma, n)$ resonances were in fact real.

One primary non-physical contribution to the cross section is introduced by the Least structure analysis. The resonance peaks are smoothed In accordance with the resolution and are not as sharp as the true physical resonance peak. This smoothing process also translates a steep rise or fall in the cross section into a less steep slope and an overshoot. This effect can cause negative solutions on either side of a resonance. This effect is a distortion which is characteristic of this type of analys is (52).

This distortion is evident in the cross section solutions for this experiment. Each of the $(\gamma, n p)$ curves has a small negative dip preceding the first resonance. The ${ }^{74} \mathrm{Ge}(\gamma, p)$ cross section has dips above and below the main peaks. Finally the ${ }^{70} \mathrm{Ge}(\gamma, n)$ result has a large negative undershoot after the primary resonance.

\section{Comparison to Other Germanium Data}

\section{The companion experiment}

In Figures 11 and 12 we present the results of an experiment performed by H. J. Vander Molen at this laboratory. These $(\gamma, n)$ and $(\gamma, 2 n)$ cross sectlons were measured by counting directly the neutrons produced in each reaction. Separated isotopes of ${ }^{70} \mathrm{Ge},{ }^{72} \mathrm{Ge},{ }^{74} \mathrm{Ge}$ and ${ }^{76} \mathrm{Ge}$ were used for targets. Detalls of the experiment and cross section results have been glven by Vander Molen (31). 


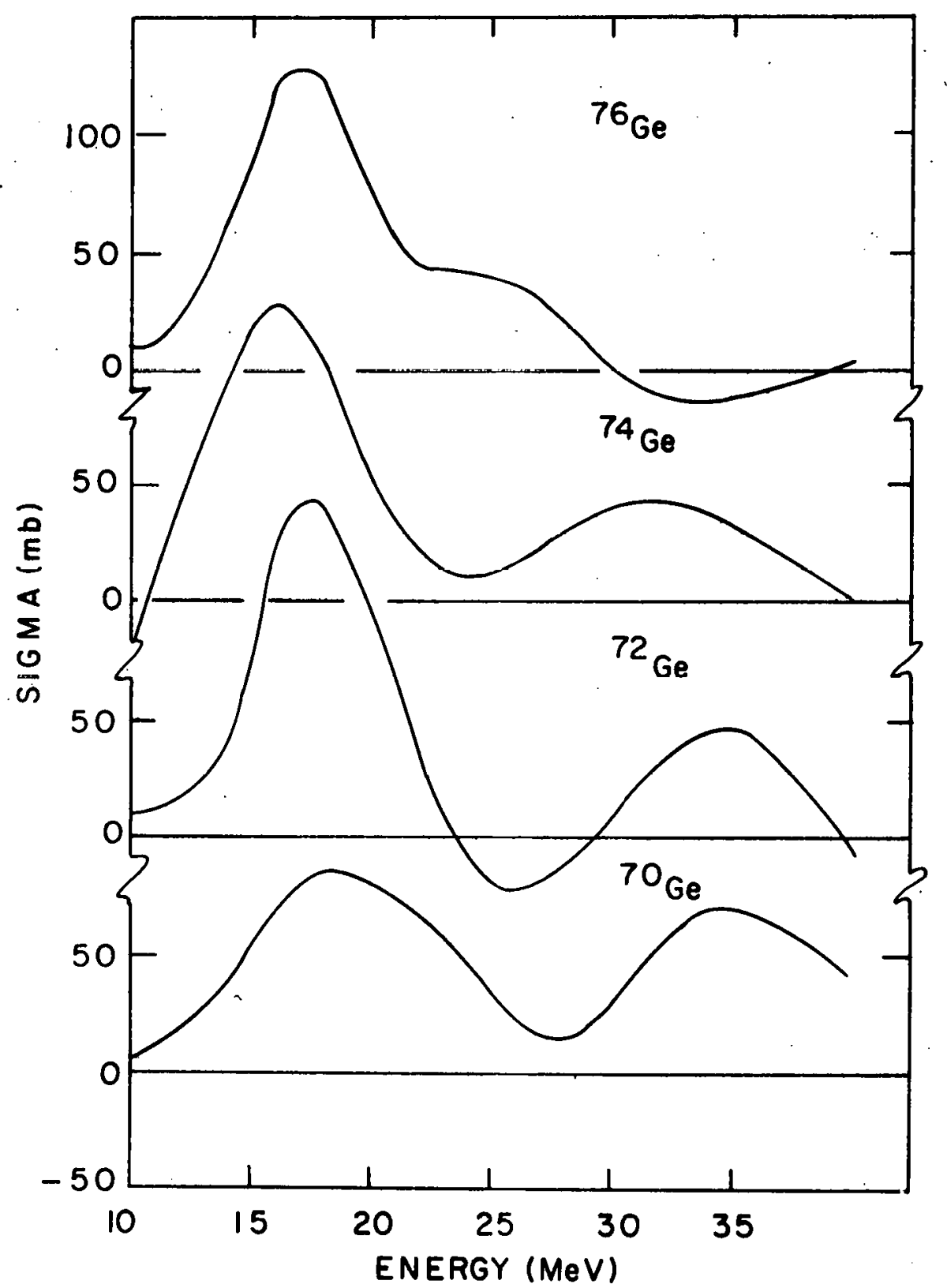

Figure 11. The $(\gamma, n)$ cross sections of ${ }^{70} \mathrm{Ge},{ }^{72} \mathrm{Ge},{ }^{74} \mathrm{Ge}$ and ${ }^{76} \mathrm{Ge}$ measured by H. J. Vander Molen 


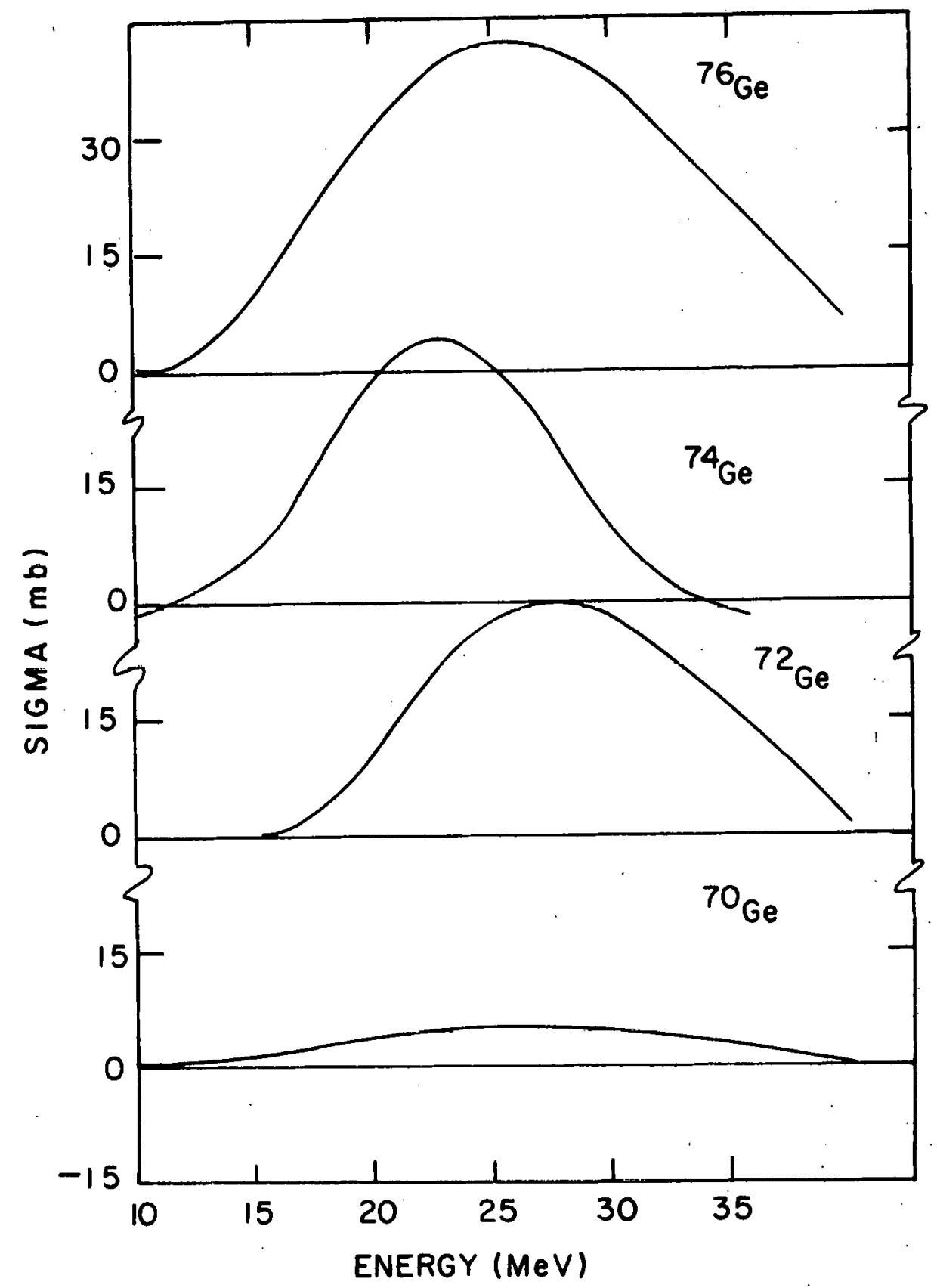

Figure 12. The $(r, 2 \mathrm{n})$ cross sections of ${ }^{70} \mathrm{Ge},{ }^{72} \mathrm{Ge},{ }^{74} \mathrm{Ge}$ and ${ }^{76} \mathrm{Ge}$ measured by $H$. J. Vander Molen 
The $(\gamma, n)$ cross sections show two distinct resonances. A main peak corresponding to the usual Giant Dipole Resonance is found at about 17 $\mathrm{MeV}$, whlle a secondary resonance is observed at higher energies. This secondary peak appears at an energy which is highest for ${ }^{70} \mathrm{Ge}$ and which decreases to a minimum in ${ }^{76} \mathrm{Ge}$. These $(\gamma, n)$ results for ${ }^{70} \mathrm{Ge}$ and ${ }^{76} \mathrm{Ge}$ agree with the activation measurements to within the experimental resolution. The striking observation is that the second resonance appears in all $(\gamma, n)$ cross sections and appears to contain systematic behavior. The $(r, 2 n)$ cross sections also show evidence of some systematic trends. The resonance peaks at about $26 \mathrm{MeV}$ in all four nuclel, and gains strength as the neutron excess is increased. Since all the $(\gamma, 2 n)$ reactions lead to stable or very long lived end products, they could not be measured in the activation work.

\section{Other germanium results}

The ${ }^{76} \mathrm{Ge}(\gamma, n)$ and ${ }^{70} \mathrm{Ge}(\gamma, n)$ reactions have been previously studied by Borello et al. (67). They report a cross section peak at $20.5 \mathrm{MeV}$ with peak height $125 \mathrm{mb}$ for ${ }^{70} \mathrm{Ge}$, and a peak at about $19 \mathrm{MeV}$ in ${ }^{76} \mathrm{Ge}$ with a helght of $249 \mathrm{mb}$. Both resonance energies are quite high in comparison to other measurements. This is probably due to the fact that the unfolding process was hampered by lack of data above $22 \mathrm{MeV}$. These results are compared with this experiment and Vander Molen's in Figures 13 and 14 .

Ferrero et al. (68) have measured the $(\gamma, n)$ and $(\gamma, n p)$ cross sections for ${ }^{70} \mathrm{Ge}$ to $31 \mathrm{MeV}$. Figures 13 and 15 compare these results to the present experiment, and to those of Vander Molen for ${ }^{70} \mathrm{Ge}$. Ferrero reports a peak at $18 \mathrm{MeV}$ in agreement with Vander Molen. These results are higher 


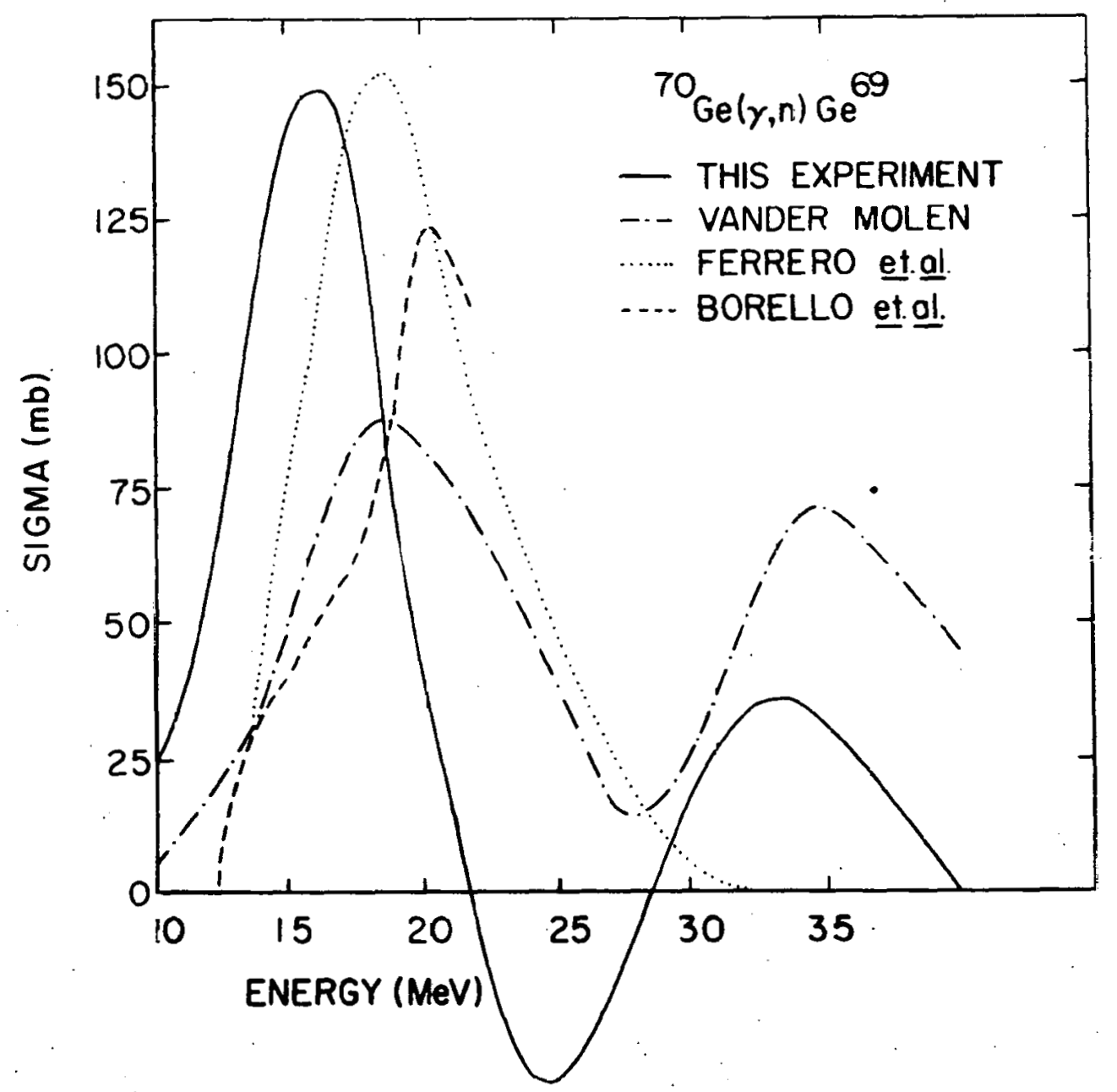

Figure 13. Comparison of the ${ }^{70} \mathrm{Ge}(\gamma, n)^{69} \mathrm{Ge}$ cross section to other data 


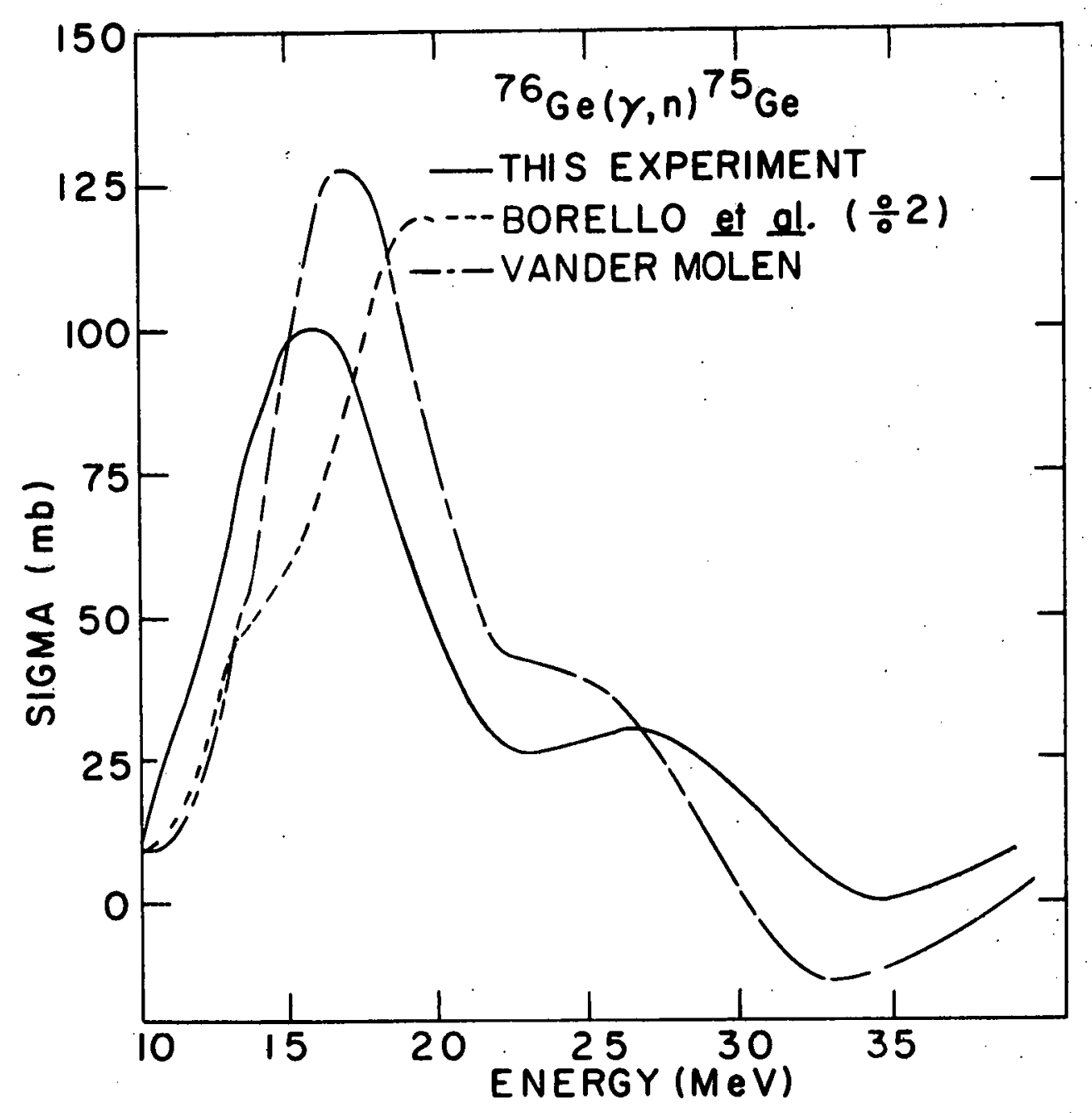

Figure 14. Comparison of the ${ }^{76} \mathrm{Ge}(\gamma, n)^{75} \mathrm{Ge}$ cross section to other data 


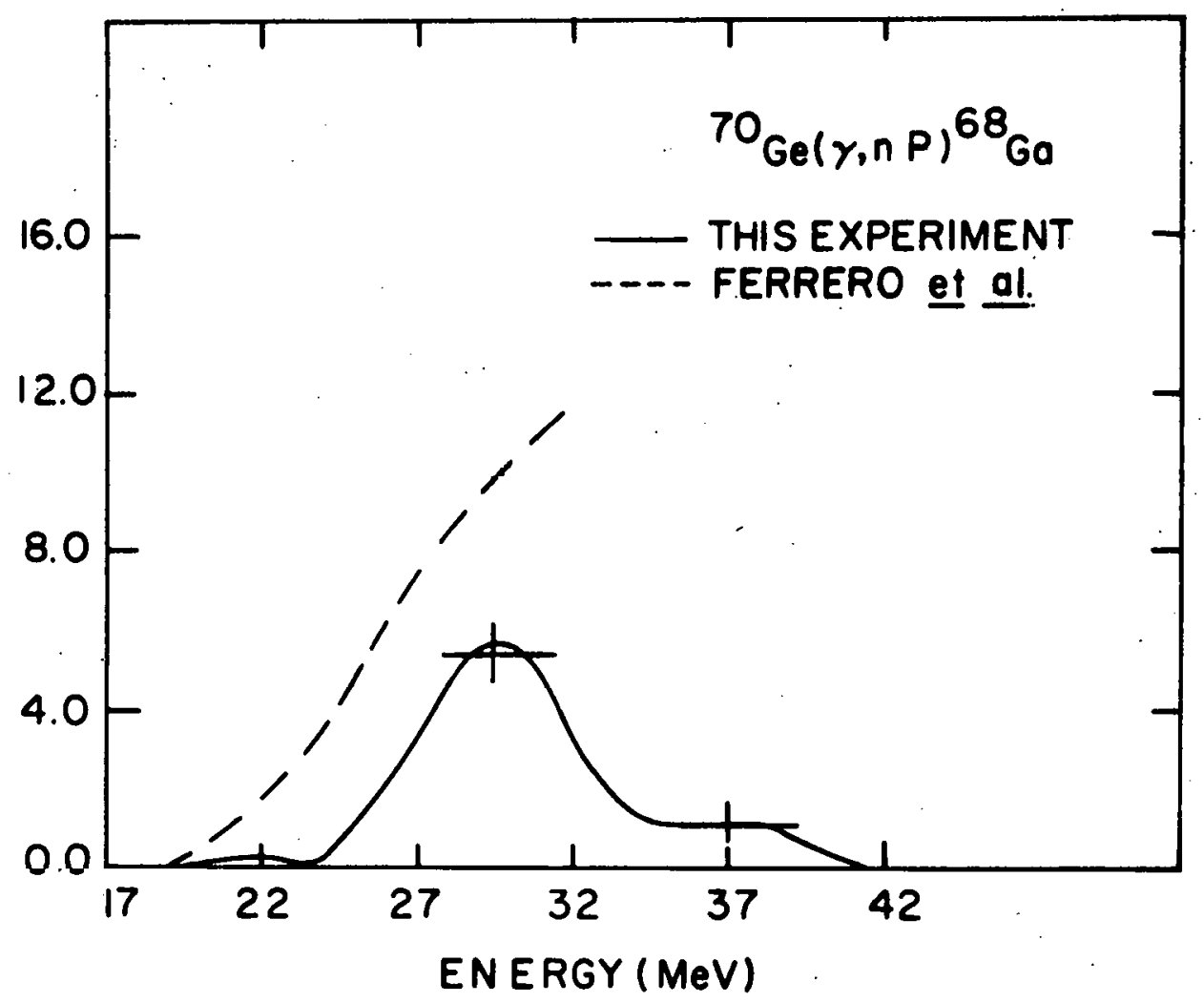

Figure 15. Comparison of the ${ }^{70} \mathrm{Ge}(\gamma, \mathrm{np}){ }^{68} \mathrm{Ga}$ cross sectlon to the cross section of Ferrero et al. 
than our result $(16.0 \mathrm{MeV})$ but are within the combined resolution for Vander Molen ( $2 \mathrm{MeV}$ ) and the present experiment ( $\mathrm{MeV}$ ). Ferrero gives a peak of about $150 \mathrm{mb}$ in good agreement with our value of $150 \mathrm{mb}$ and the Borello value of $125 \mathrm{mb}$. Vander Molen's result is somewhat smaller, about $85 \mathrm{mb}$, but this value is probably effected by the marginal quality of his data for ${ }^{70} \mathrm{Ge}$ (31). The ${ }^{70} \mathrm{Ge}$ yield curve measured by Vander Molen was the first curve measured in that experiment and was of extremely poor statistical quality, the worst of all his data. The $(\gamma, n p)$ results do not agree well. Ferrero reports a strength about twice that found in this experiment. It is disturbing that there is not evidence of the resonance at $29 \mathrm{MeV}$. It should be noted, however, that the results of Ferrero et al. (68) also fall to reproduce a.resonance in the ${ }^{32} s(r, n p)$ cross section at $26 \mathrm{MeV}(69)$.

\section{Total Absorption Cross Sections}

Total cross sections for photon absorption by ${ }^{70} \mathrm{Ge},{ }^{72} \mathrm{Ge},{ }^{74} \mathrm{Ge}$ and ${ }^{76}$ Ge have been prepared by combining the results of this experiment with those of Vander Molen (31) and are presented in Figure 16. The partial cross sections for each reaction have been summed to determine the total contribution at each energy. The vertical error bars denote the magnitude of the total experimental error while the horizontal bars indicate the curve resolution as described previously. The error in absolute cross section normalization has an average value of $15 \%$ for all curves.

since the results of the two experiments for the ${ }^{76} \mathrm{Ge}(\gamma, n)$ cross section agree to within the experimental errors in normalization, no at tempt has been made to renormalize the results of either experiment. 


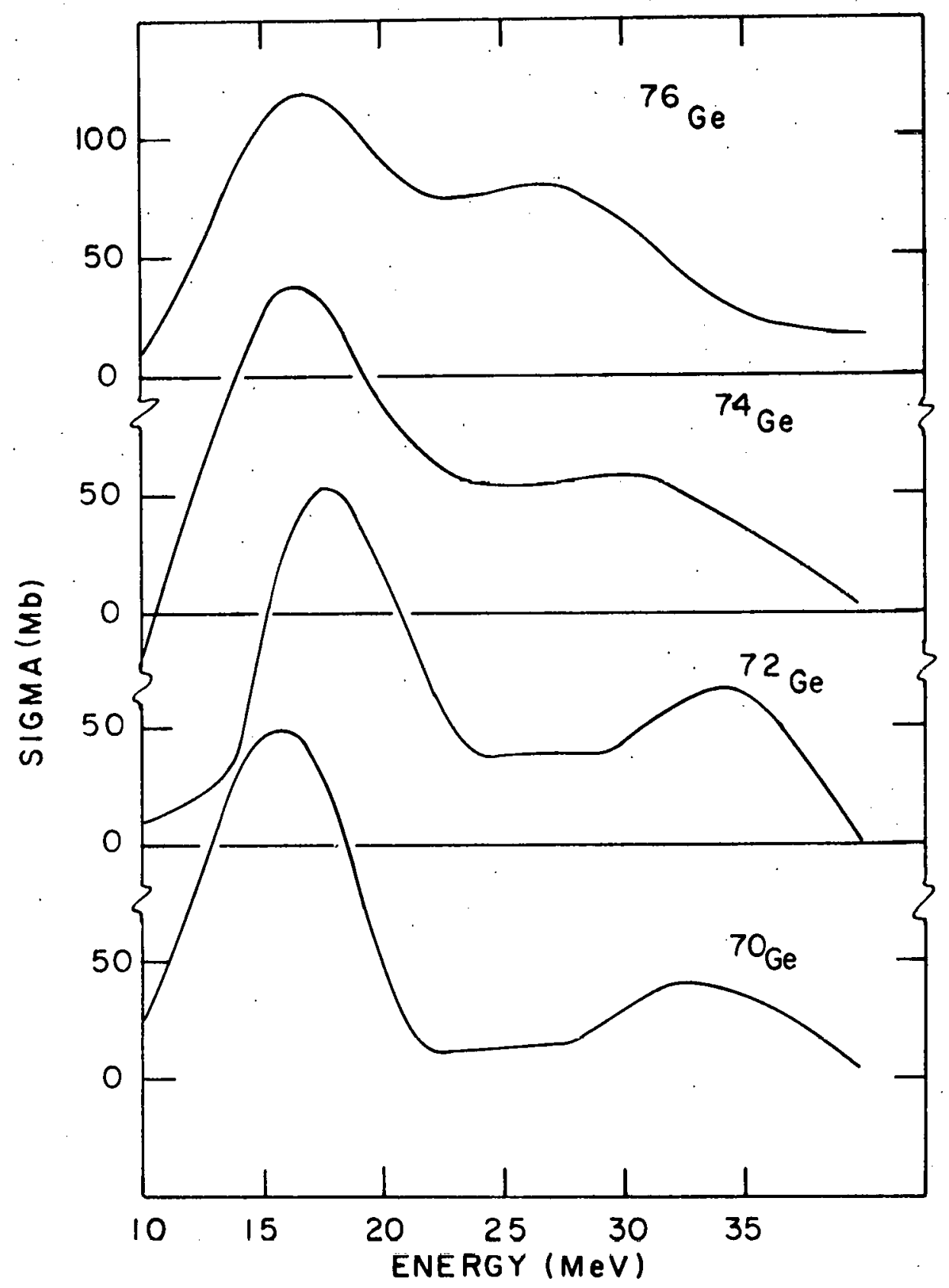

Figure 16. Total absorption cross sectlons for ${ }^{70} \mathrm{Ge},{ }^{72} \mathrm{Ge}$, ${ }^{74} \mathrm{Ge}$, and ${ }^{76} \mathrm{Ge}$ 
The ${ }^{70} \mathrm{Ge}$ and ${ }^{76} \mathrm{Ge}$ totals have been prepared using the $(\gamma, n)$ results from the activation experiment since the experimental resolution of the activation measurement was better in both instances, and the statistical quality of the ${ }^{70} \mathrm{Ge}$ data was better in the activation work. The $(\gamma, p)$ contribution in ${ }^{70} \mathrm{Ge},{ }^{72} \mathrm{Ge}$, and ${ }^{76} \mathrm{Ge}$ was estimated by including the measured ${ }^{74}(\gamma, p)$ result in each total curve. The negative undershoots in ${ }^{70} \mathrm{Ge}(\gamma, n)$ and ${ }^{72} \mathrm{Ge}(\gamma, n)$ were not summed into the total, and this gives the flat appearance to those curves around $25 \mathrm{MeV}$.

The most unusual feature of the total cross sectlons is the large amount of strength in the resonance at high excitation energies. This secondary resonance is due almost entirely to contributions from the single and double neutron cross sections, since the measured $(\gamma, p)$ and $(r, n p)$ contributions are quite small in all isotopes.

The secondary resonance exhibits two systematic features. First, we observe that as the neutron excess increases, the peak position decreases from about $34 \mathrm{MeV}$ in ${ }^{70} \mathrm{Ge}$ to about $27 \mathrm{MeV}$ in ${ }^{76} \mathrm{Ge}$. Secondly, the strength in the secondary resonance increases by nearly a factor of two between ${ }^{70} \mathrm{Ge}$ and ${ }^{72} \mathrm{Ge}$, and then remains constant within the experimental precision. Further discussion of both these trends is delayed until Chapter VI. 


\section{CHAPTER VI. DISCUSSION AND CONCLUSIONS}

The measurement of the $(\gamma, n),(\gamma, 2 n)$ and $(\gamma, n p)$ cross sections in ${ }^{70} \mathrm{Ge},{ }^{72} \mathrm{Ge},{ }^{74} \mathrm{Ge},{ }^{76} \mathrm{Ge}$ and the $(\gamma, \mathrm{p})$ cross section in ${ }^{74} \mathrm{Ge}$ make it possible to construct a nearly complete description of the photodisintegration of these nuclei. It is possible to identify with confidence several systematic effects in the measured cross sections. The first section of this chapter discusses isospin effects in the decay of dipole state of germanium. The second section is a discussion of systematic trends observed in the four total absorption cross sectlons. The last section puts forth suggestions for future work derived from the present work.

\section{Isospin Effects}

For many medium-heavy nuclel, the $(r, n p)$ reaction is particularly interesting from an isospin viewpoint. 'Brlefly, neutron emission from the $T$, glant resonance directly to the ground state of the residual nucleus is isospin forbidden. However, neutron emission from $T$, glant resonance state is isospin allowed to excited states of the proper isospin, $T_{>} \pm 1 / 2$, in the residual nucleus. The first such state is the Isobaric analog to the ground state of the nucleus populated by proton emission. The excitation energy of this level generally lies very close to the last proton binding energy. Since neutron decays are not influenced by the Coulomb barrier, a sizable fraction of the $T_{>}$giant resonance strength should appear in decays through these analog levels.

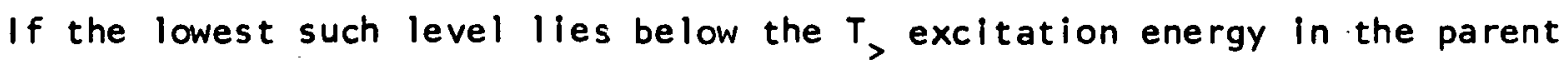
nucleus, and is also unstable to proton decay, the $(\gamma, n p)$ cross section 
should be enhanced by the $T_{>}$giant resonance component.

The $(\gamma, n p)$ cross sections from ${ }^{70} \mathrm{Ge},{ }^{72} \mathrm{Ge}$, and ${ }^{76} \mathrm{Ge}$ clearly reflect the operation of these isospin selection rules in the decay of the dipole state. The energy and isospin systematics of the $(\gamma ; n p)$ channel for these nuclel are shown in Figure 17.

Figure 17 shows the excitation energies of the glant resonance components, the $(\gamma, n p)$ thresholds, and the first analog states in the $(\mathrm{Z}, \mathrm{N}-\mathrm{l})$ residual nuclel. The glant resonance components were found by adding the splitting energies predicted by Leonardi (40) to a $T_{<}$energy assumed to be the energy of the first peak of the $(\gamma, \pi)$ cross section. The threshold energies were obtained from nuclear data sheets. The energies of the analog states were obtained from measured values when possible, or were calculated from the formula of Janecke (44).

In ${ }^{70} \mathrm{Ge}$, neutron decay to the $T=7 / 2$ level in ${ }^{69} \mathrm{Ge}$ is energetically allowed, but this state is bound with respect to proton decay by $0.6 \mathrm{MeV}$. The excitation energy of this $T=7 / 2$ state has been measured (54) and the value of the $7 \mathrm{MeV}$ (relative to the ${ }^{69} \mathrm{Ge}$ ground state) is in exact agreement with the calculated value. This means that $T_{>}$strength feeding this level will appear in the $(\gamma, n)$ channel, rather than the $(\gamma, n p)$. This . $T_{>}$contribution to the single neutron cross section should produce a

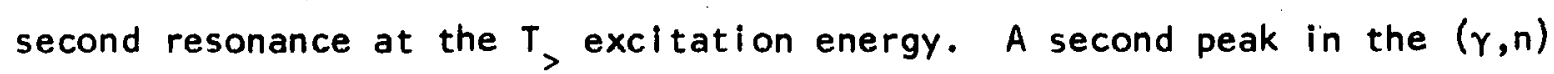
cross section of ${ }^{64} \mathrm{Zn}$ at the proper $T_{>}$energy has been reported by Schamber (28), but the resolution of the present measurement on ${ }^{70} \mathrm{Ge}$ prohibits observation of any simllar structure. Schamber also measured the ${ }^{64} \mathrm{Zn}(\gamma, \mathrm{np})$ cross section and it is interesting to compare it to the 
Figure 17. Energy and isospin systematics of the $(\gamma, n p)$ channel 


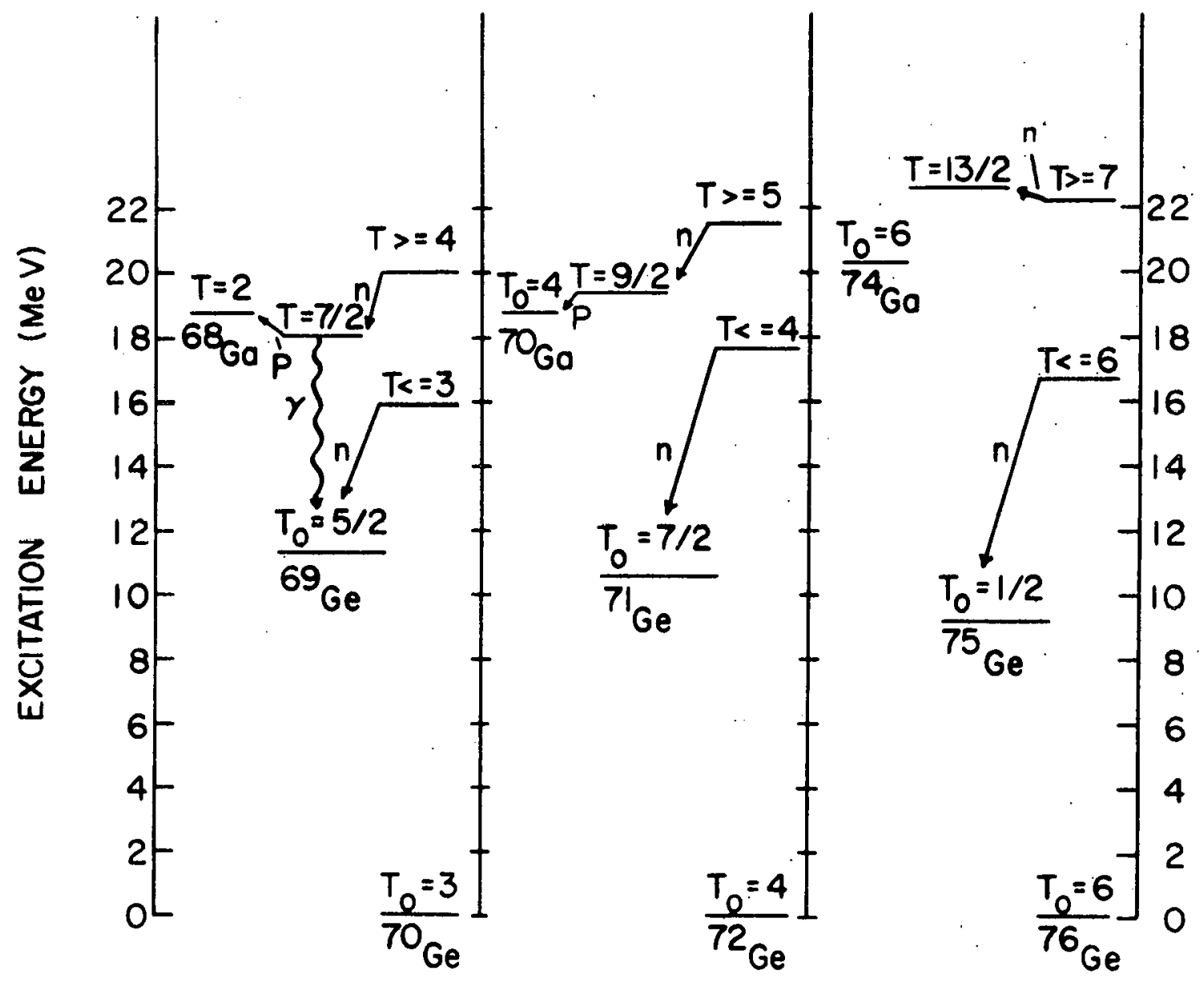


${ }^{70} \mathrm{Ge}(\gamma, \mathrm{np})$ result. In both $(\gamma, \mathrm{np})$ results, there is no evidence for any strength at the excitation energy of the $T_{>}$giant resonance, implying (on the basis of energy systematics) that the $T_{>}$decay contributes to the $(\gamma, n)$ cross section. In both the ${ }^{64} \mathrm{Zn}(\gamma, n p)$ and ${ }^{70} \mathrm{Ge}(\gamma, n p)$ cross sections the greatest strength appears in a resonance located at an excitation

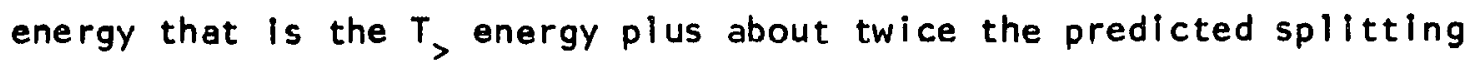
energy of the $T_{<}$and $T_{>}$giant resonance components.

in ${ }^{72} \mathrm{Ge}$, the $T=9 / 2$ analog level is not proton bound, and $T_{>}$contributions to the $(\gamma, n p)$ cross section are both isospin and energy allowed. One expects to see enhancement of the $(\gamma, n p)$ cross section near

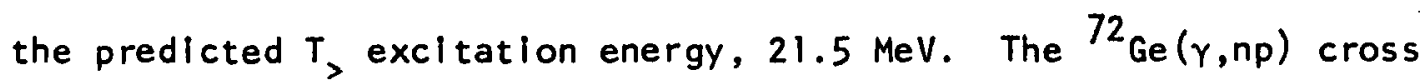
section measured in this experiment displays a prominent resonance at $21.8 \mathrm{MeV}$, that dominates the entire cross section and is not seen in ${ }^{70} \mathrm{Ge}(r, n p)$. Clearly this is the allowed contributions from the decay of the $T_{>}$glant resonance.

For ${ }^{76} \mathrm{Ge}$, the first analog level lies slightly above the center of the $T_{>}$giant resonance in energy. While the $T_{>}$state is probably broad enough to overlap with the analog state, the high energy of the analog state should certainly reduce neutron decays from the $T$, giant resonance. Indeed, this is the observed effect. While there is some indication of

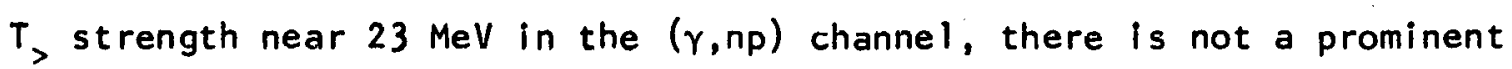

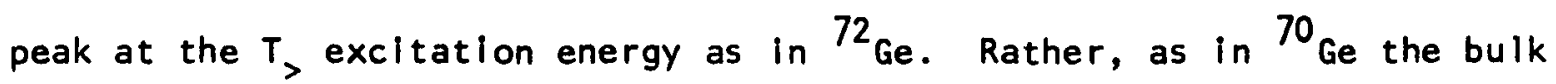
of the cross section is at 29 and $35 \mathrm{MeV}$.

We have Identified the peak at $21.8 \mathrm{MeV}$ in the ${ }^{72} \mathrm{Ge}(\gamma, \mathrm{np})$ cross section as contributions from the $T_{>}$giant resonance. If we place the 
$T_{<}$glant resonance at the peak energy of ${ }^{72} \mathrm{Ge}(\gamma, n)$ cross section $(17.5$ $\mathrm{MeV})$ we can estimate the splitting of the two glant resonance components as $4.3 \mathrm{MeV}$. This value is in excellent agreement with the predicted values of $4.0 \mathrm{MeV}$ by Leonardi (40) and $4.2 \mathrm{MeV}$ by Akyuz and Faillieros (22). Not all of the $T_{>}$giant resonance strength is expected to contribute to the $(\gamma, n p)$ cross section. In particular, $T_{>}$contributions (and $T_{<}$as well) are expected to be found in the $(\gamma, p)$ reaction cross section. A measure of the size of the $T_{>}$contribution to the $(\gamma, n p)$ reaction is the ratio of $\sigma_{-1}\left(T_{>}\right)$to $\sigma_{-1}\left(T_{<}\right)$calculated from the $(\gamma, n p)$ cross section for $T_{>}$and the $(\gamma, n)$ cross section for $T_{<}$strength. For ${ }^{72}$ Ge we find the value

$$
\sigma_{-1}\left(T_{>}\right) / \sigma_{-1}\left(T_{<}\right)=0.032
$$

This ratio can be predicted from the isospin sum rule calculations, and Leonardi (40) gives a value of 0.17 for ${ }^{72} \mathrm{Ge}$. Hayward et al. (43) predict a lower value of 0.12 . While the experimental value is low, we note that $T_{>}$strength in the proton decay channel has not been included in this estimate, while the sum rule estimates the entire $T_{>}$contribution. The measurement of the ${ }^{74} \mathrm{Ge}(\gamma, \mathrm{p})$ cross section is of interest in determining the $T_{>}$contributions to proton decay, and is the subject of the next section of this chapter.

The ${ }^{74} \mathrm{Ge}(\gamma, \mathrm{p})$ cross section

The role of isospin in the decay of the dipole state in germanium is also reflected in the ${ }^{74} \mathrm{Ge}(\gamma, p)$ reaction cross section. The $(\gamma, p)$ cross section shows resonant structure at 18,24 and $30 \mathrm{MeV}$. The behavior at $18 \mathrm{MeV}$ and $24 \mathrm{MeV}$ is easily explained if the $T_{<}$and $T_{>}$resonances are 
located at these energies. The enhancement at $24 \mathrm{MeV}$ in the $(\gamma, p)$ cross section is due to the isospin selection rules, which forbid neutron decay from the $T_{>}=6$ states in ${ }^{74} \mathrm{Ge}$ to the $T_{<}=9 / 2$ states in ${ }^{73} \mathrm{Ge}$, but permit proton decay to either the $T_{>}=11 / 2$ and $T_{>}=13 / 2$ states in ${ }^{73} \mathrm{Ga}$. Neutron decay to $T=11 / 2$ states in ${ }^{73} \mathrm{Ge}$ is allowed. The first such state is predicted to be at $20.8 \mathrm{MeV}$ and is proton unstable. As in ${ }^{72} \mathrm{Ge} \mathrm{T}_{\text {, con- }}$ tributions should enhance the ${ }^{74} \mathrm{Ge}(\gamma, \mathrm{np})$ cross section.

The first peak in the $(\gamma, n)$ cross section for ${ }^{74}$ Ge should occur at the energy of the $T_{<}$glant resonance. Vander Molen (31) finds this at $17 \mathrm{MeV}$, and does not see a second resonance at the $T_{>}$energy as Schamber does in ${ }^{64} \mathrm{Zn}$. This is consistent with the fact that the $(\gamma, n p) T_{>}$contribution is energetically allowed in ${ }^{74} \mathrm{Ge}$ (as in ${ }^{72} \mathrm{Ge}$ ) but is not in ${ }^{64} \mathrm{Zn}$.

A curious feature is that the $(\gamma, n)$ and $(\gamma, p)$ cross section do not appear to peak at the same energles. This feature was also noted in Clark's analys ls of ${ }^{64} \mathrm{Zn}(30)$, and is probably due to uncertainties in energy calibrations of the experiments.

The strength at $30 \mathrm{MeV}$ is at nearly the same energy as the secondary resonance observed in the ${ }^{74} \mathrm{Ge}$ total cross section. Clark also observed a higher energy component in the ${ }^{64} \mathrm{Zn}(\gamma, \mathrm{p})$ cross section and has tentatively identified that strength as the electric quadrupole giant resonance, on the basis of angular correlation results. It is doubtful that this explanation will suffice in the case of ${ }^{74} \mathrm{Ge}$. The combined strength of the $(\gamma, p)$ and $(\gamma, n)$ channels at $30 \mathrm{MeV}$ is much greater than the $8 \%$ of the total cross section predicted for the quadrupole resonance. 
Assigning the $T_{<}$resonance at $18 \mathrm{MeV}$ and the $T_{>}$resonance at $24 \mathrm{MeV}$ gives an isospin splitting energy of $E=6.0 \pm 1.0 \mathrm{MeV}$ for ${ }^{74} \mathrm{Ge}$, in good agreement to the predicted values of $5.4 \mathrm{MeV}$ (40) and $5.0 \mathrm{MeV}$ (22).

An attempt has been made to fit two curves to the $(\gamma, p)$ cross section, and compute the bremsstrahlung welghted cross section for each resonance. The $(\gamma, n){ }^{74} \mathrm{Ge}$ curve has also been fitted and $\sigma_{-1}$ computed. The $\sigma_{-1}\left(T_{<}\right)$ result was estimated as the sum of $\sigma_{-1}(\gamma, n)$ and $\sigma_{-1}$ for the first $(\gamma, p)$ resonance. The value of $\sigma_{-1}\left(T_{>}\right)$was taken as the second $(\gamma, p)$ resonance. The ratio of results is

$$
\sigma_{-1}\left(T_{>}\right) / \sigma_{-1}\left(T_{<}\right)=0.038
$$

As indlcated earlier $\mathrm{T}_{>}$strength is expected to be found in the $(\gamma, n p)$ cross section.

Sum rule calculations can again be used to estimate the ratio of the entire $T_{>}$contribution to the $T_{<}$strength. Leonardi (40) finds a value of 0.13 , while Hayward et al. give their value as 0.09 . The low value of the experimental value indlcates we should expect $T_{>}$contribution in the ${ }^{74} \mathrm{Ge}(\gamma, \mathrm{np})$ cross section. Considering the crudeness of our experimental estimate, agreement with sum rule results in good.

\section{Systematic Effects in the Total Cross Sections}

The total absorption cross sections from all the reactions observed in this series of experiments, presented in the preceding chapter, have been fitted by least squares methods to a function that represents the sum of two Lorentz line shapes, and has the following functional form; 


$$
\sigma_{\text {tot }}(E)=\frac{\sigma_{a}^{0}\left(E \Gamma_{a}\right)^{2}}{\left(E_{a}^{2}-E^{2}\right)^{2}-E^{2} \Gamma_{a}^{2}}+\frac{\sigma_{b}^{0}\left(E \Gamma_{b}\right)^{2}}{\left(E_{b}^{2}-E^{2}\right)^{2}+E^{2} \Gamma_{b}{ }^{2}}
$$

Goodness of fit was judged by values for the chi-squared parameter, defined as

$$
x^{2}=\frac{1}{n} \sum_{i=1}^{n} \frac{\left(\sigma_{1}-\sigma_{1 F I T}\right)^{2}}{\left(\Delta \sigma_{i}\right)^{2}}
$$

where $x^{2}=1$ is the optimum value. Values of all parameters for each curve are given in Table 8 , and the fitted curves are compared to the experimental data in Figures 18, 19, 20 and 21 . The error In absolute normalization of experimental cross sections is about $20 \%$.

\section{Giant resonance systematics}

The energy of the main resonance remains constant within the experimental resolution for all nuclei. The energy of the second resonance, however, decreases in energy systematically from ${ }^{72} \mathrm{Ge}$ to ${ }^{76} \mathrm{Ge}$. The FWHM of both resonances increases as neutrons are added, with the only exception being the second resonance in ${ }^{76} \mathrm{Ge}$.

The fact that the glant resonance energy remains essentially constant is in agreement $w$ th collective model treatments which predict a slow $A$ dependence of the resonance energy. Furthermore, the energies found here are in agreement with measurements on nearby nuclei $(7,26,28,35,67,68)$.

The increasing FWHM of the main giant resonance with increasing neutron excess is in qualitative agreement with predictions of the dynamic collective model. Addition of neutrons makes the nuclear surface 
Table 8. Fltted parameters of total cross sections of germanium ${ }^{a}$

\begin{tabular}{lcccccc}
\hline Nucleus & $\begin{array}{c}\sigma_{a}^{0} \\
(\mathrm{mb})\end{array}$ & $\begin{array}{c}\Gamma_{a} \\
(\mathrm{MeV})\end{array}$ & $\begin{array}{c}\mathrm{E}_{\mathrm{a}} \\
(\mathrm{MeV})\end{array}$ & $\begin{array}{c}\sigma_{b}{ }^{\circ} \\
(\mathrm{mb})\end{array}$ & $\begin{array}{c}\Gamma_{b} \\
(\mathrm{MeV})\end{array}$ & $\begin{array}{c}E_{b} \\
(\mathrm{MeV})\end{array}$ \\
\hline${ }^{70} \mathrm{Ge}$ & 150 & 5.8 & 15.5 & 41.3 & 5.74 & 33.0 \\
$7{ }^{7 \mathrm{Ge}}$ & 150 & 5.9 & 17.5 & 66.0 & 8.5 & 34.0 \\
$74 \mathrm{Ge}$ & 144 & 6.8 & 16.5 & 60.1 & 11.2 & 30.0 \\
$76_{\mathrm{Ge}}$ & 120 & 8.3 & 16.5 & 78.5 & 9.5 & 26.7 \\
\hline
\end{tabular}

$a_{\sigma}{ }^{0}$ is peak height in millibarns

$\Gamma$ is FWHM of resonance in MeV.

$E$ is resonance energy in MeV. 


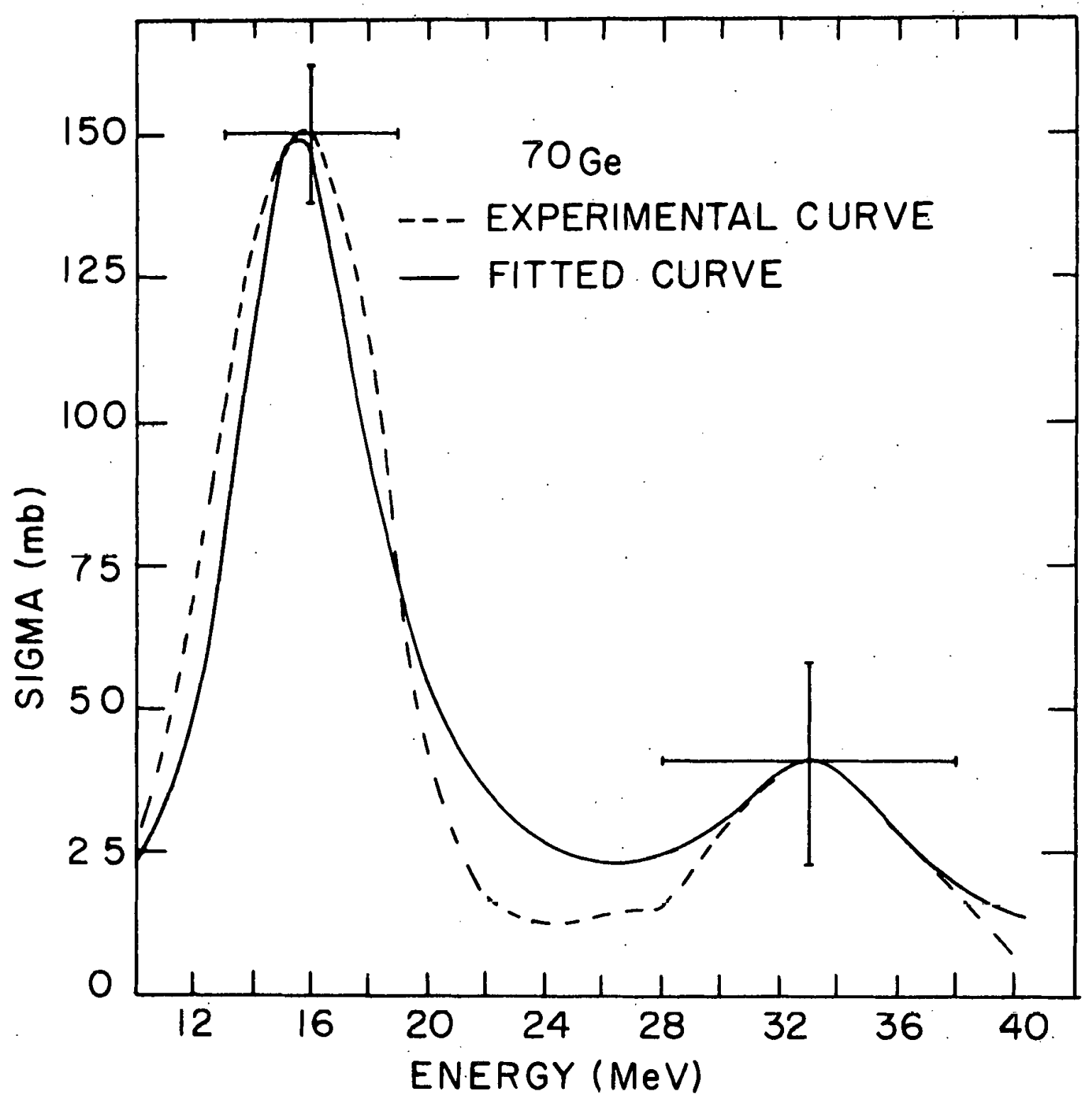

Figure 18. Comparison of experimental ${ }^{70} \mathrm{Ge}$ total cross section with fitted curve 


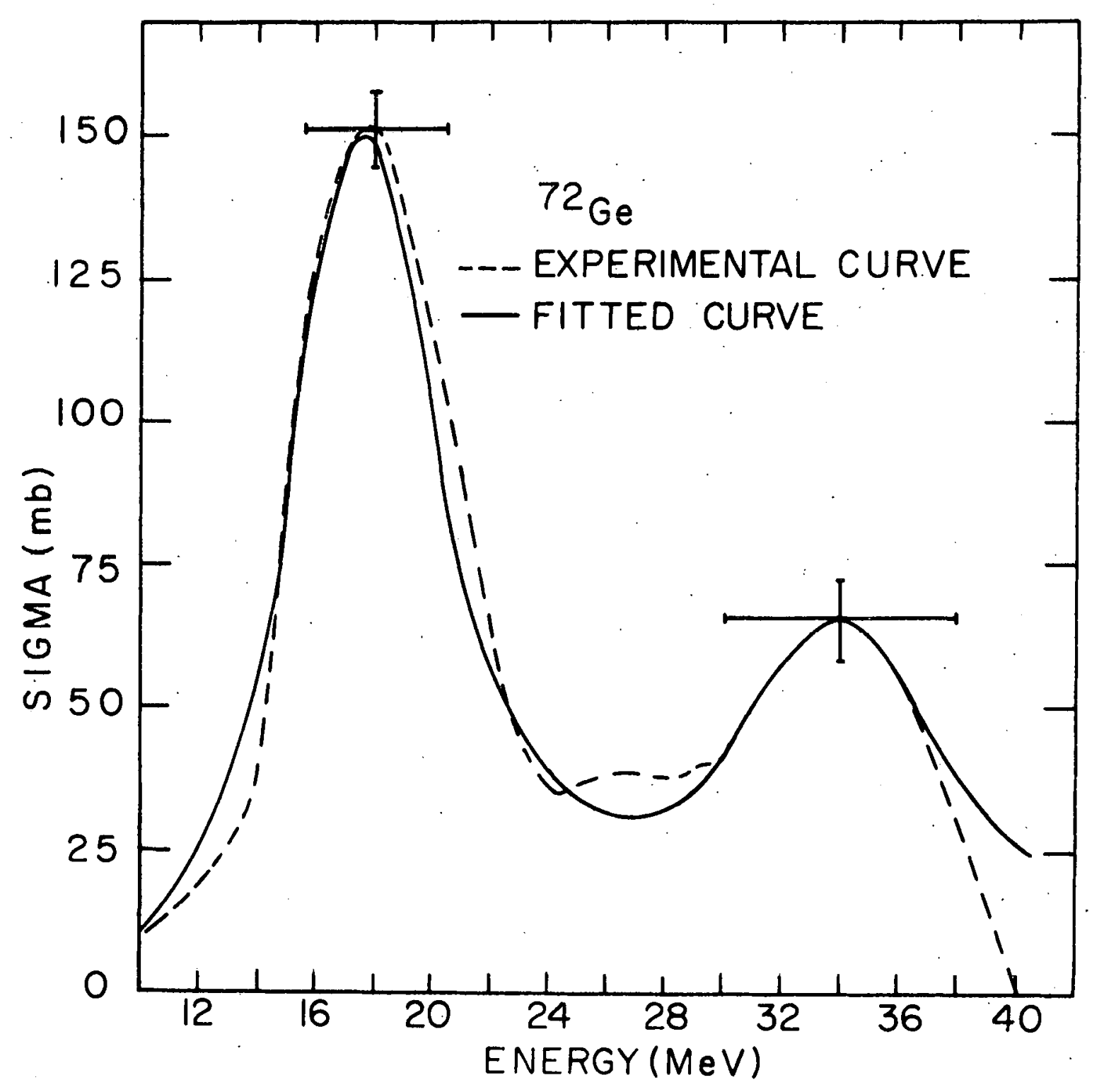

Figure 19. Comparison of experimental ${ }^{72} \mathrm{Ge}$ total cross section with fitted curve 


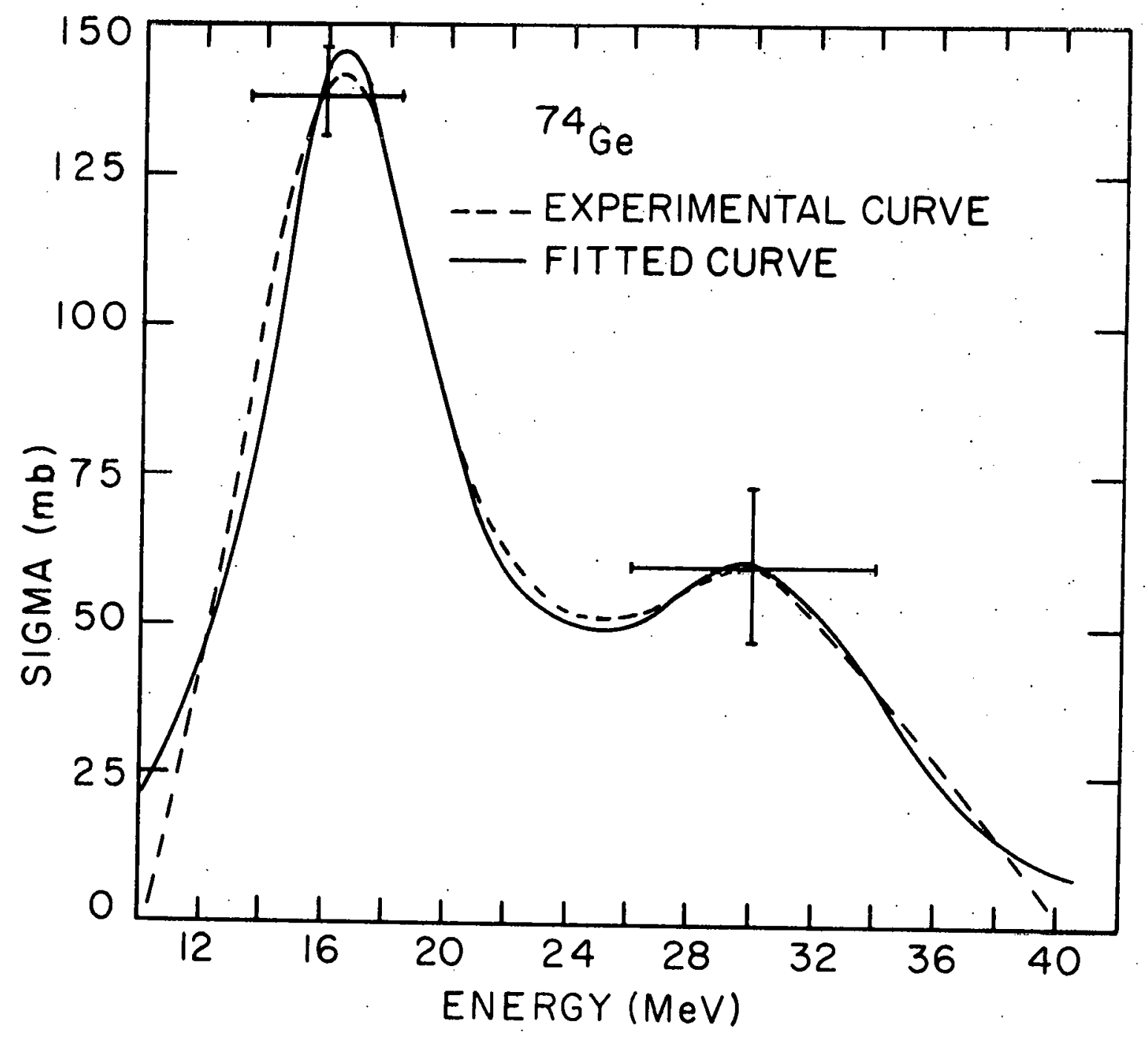

Figure 20. Comparison of experimental ${ }^{74} \mathrm{Ge}$ total cross section with fitted curve 


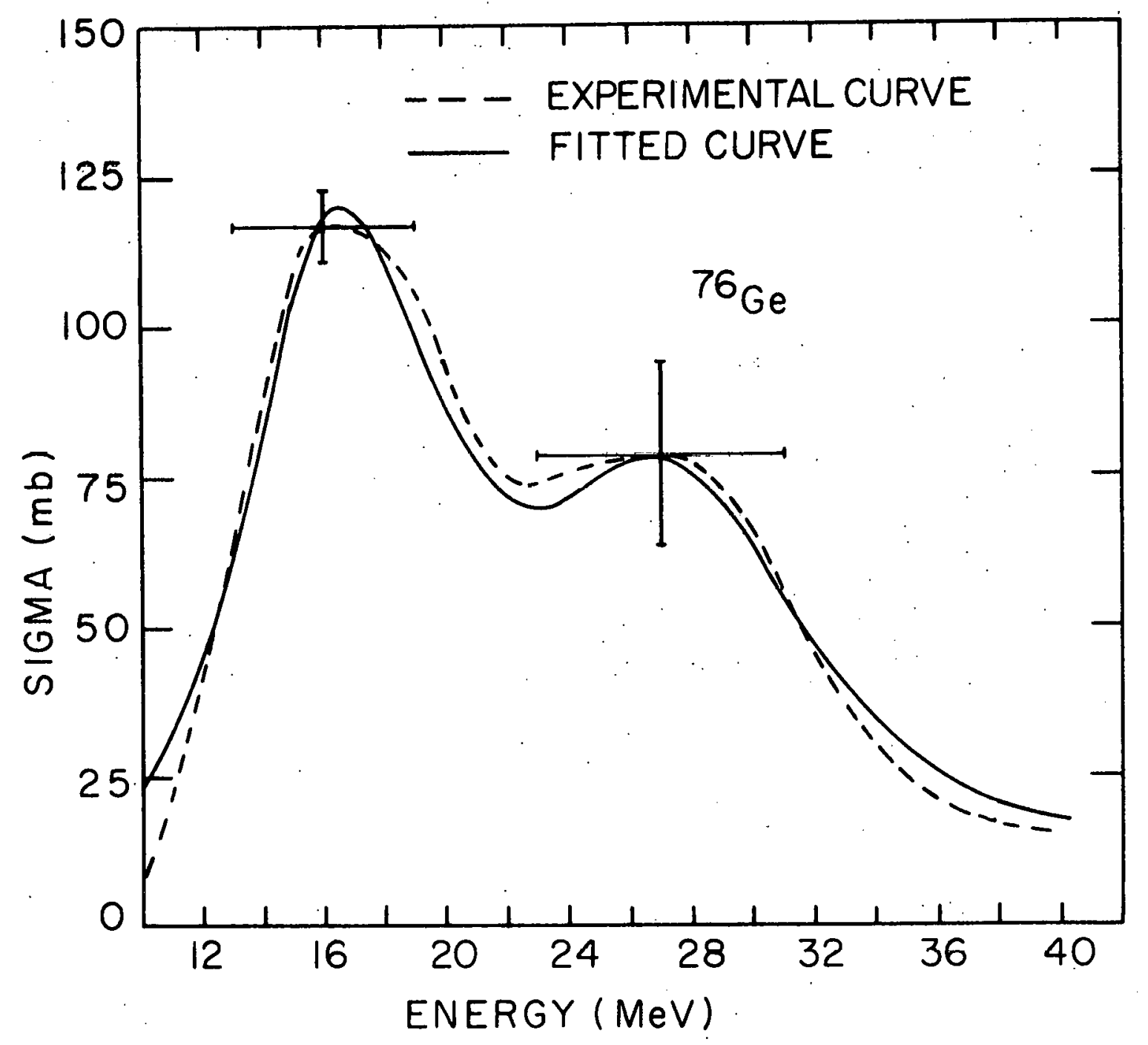
Figure 21. Comparison of experimental ${ }^{76} \mathrm{Ge}$ total cross section
with fitted curve 
"softer", strengthening the dipole-surface vibration coupling which splits the giant resonance into several components. These components appear as one broad resonance in poor resolution experiments. Owen and Mui rhead have reported a calculation by Huber for ${ }^{74} \mathrm{Ge}(70)$. Good agreement between experimental results (70) and theory are obtained in the glant resonance region. The predicted FWHM is $7 \mathrm{MeV}$, in good agreement to the $6.8 \mathrm{MeV}$ found in this work. Fielder et al. (35) have performed a similar comparison for ${ }^{75}$ As using the results of a dynamic collective model. Again a broad glant resonance is predicted and quite good agreement with experimental results is established.

Table 9 presents the cross section strengths integrated to $40 \mathrm{MeV}$ calculated by using the fitted curve parameters. The integrated cross section for the first resonance is as great or slightly exceeds the value given by the dipole sum rule (sixth column), without the addition of the exchange force term. The average of the excess integrated cross section in the main resonance over the sum rule values is approximately $15 \%$.

Structure above the glant resonance

It is evident that considerable resonant strength appears in the germanium cross sections at energies above the giant resonance. The secondary resonance contains an integrated strength that is $20 \%$ of the giant resonance strength for ${ }^{70} \mathrm{Ge}$, and about $45 \%$ of the glant resonance strength for the three other nuclei. The secondary resonance is a broad structure with a FWHM several MeV wider than that of the glant resonance. This structure appears at an energy more than twice the glant resonance energy in ${ }^{70} \mathrm{Ge}$, but this energy steadily decreases to 1.6 times the glant 
Table 9. Resonance strengths for germaniuma

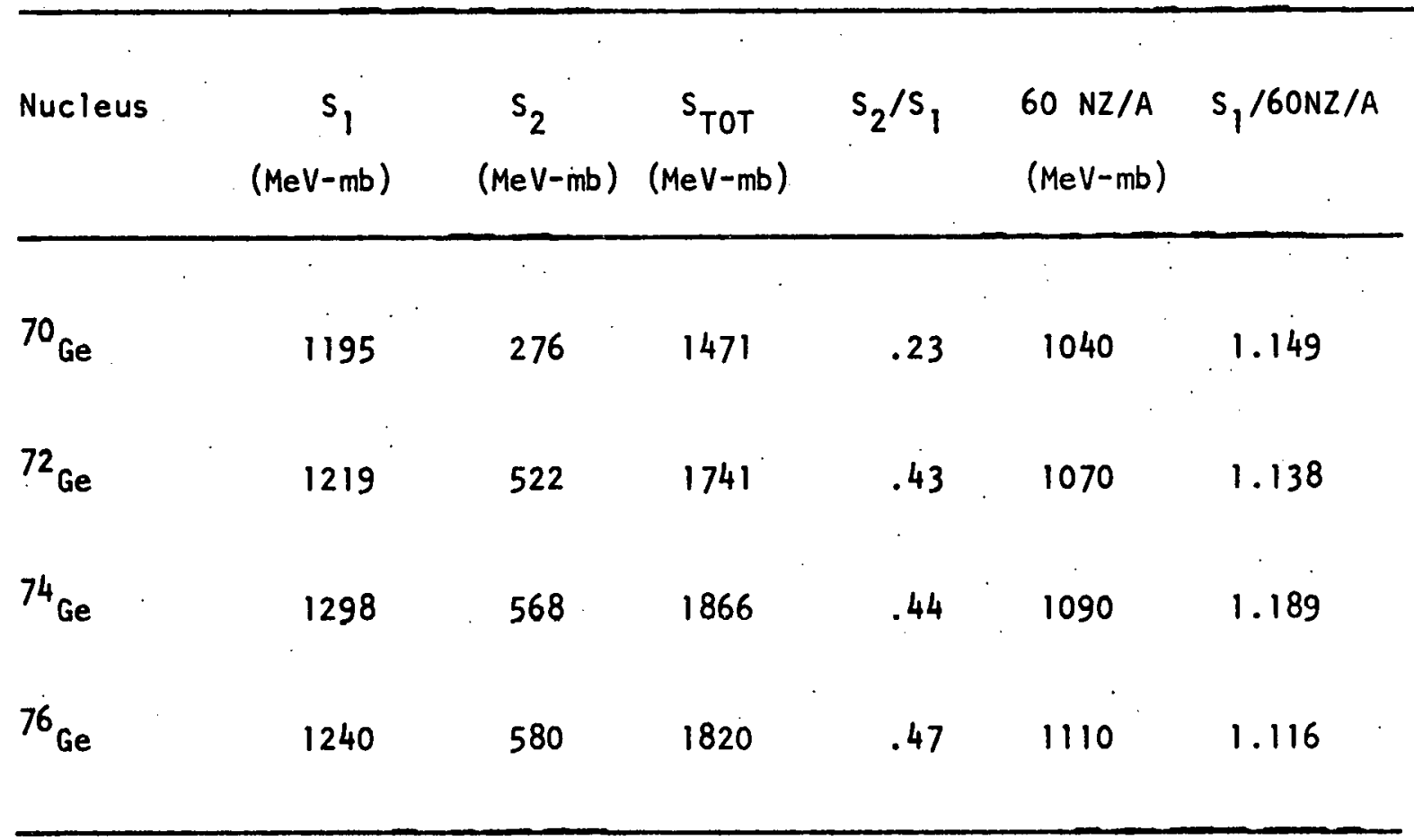

${ }^{a} s_{1}$ is the integrated cross section in the primary resonance.

$\mathrm{S}_{2}$ is the integrated cross section in the secondary resonance.

$S_{\text {TOT }}$ is the total integrated cross section. 
resonance energy in ${ }^{76} \mathrm{Ge}$.

Several mechanisms have been proposed at various times to explain structure at energies above the giant resonance. Among these mechanisms are: (a) the giant quadrupole resonance, (b) isobaric spin splitting of the giant resonance, (c) deformation splitting of the giant resonance and (d) additional dipole contributions to the cross section in the form of "overtones" to the dipole resonance. However, none of these mechanisms can reasonably account for the large secondary resonances observed here. Quadrupole contributions to the photon absorption cross section have been calculated by several authors $(71,72,73)$. The hydrodynamic model discussed in Chapter 11 predicts a quadrupole resonance at 1.6 times the energy of the electric dipole resonance, or at about $26.5 \mathrm{MeV}$ for germanium. The integrated cross section under this quadrupole resonance is estimated to be about $8 \%$ of the integrated cross section of the dipole resonance. Lushniknv and Urin (72) predict that the maximum of the quadrupole resonance occurs at an energy given by:

$$
E=190 \mathrm{~A}^{-1 / 3} \mathrm{MeV} \text {, }
$$

which gives an average resonance energy of $45 \mathrm{MeV}$ for germanium. The FWHM, $\Gamma_{Q}$, is given approximately by:

$$
\Gamma_{Q}=20 \mathrm{~A}^{-1 / 3} \mathrm{MeV} \text {. }
$$

This gives values of about $4.8 \mathrm{MeV}$ for the germanium isotopes. Clearly these predictions do not fit the resonances observed in germanium, elther in strength or energy dependence. In general, the integrated cross section in all the second resonance is far in excess of the expected 
quadrupole contribution. The width of the second resonance is nearly twice the width one expects for the quadrupole resonance, al though one suspects that dipole-quadrupole interactions will broaden the quadrupole resonance somewhat. While some structure above the glant resonance is posslbly from quadrupole contributions, a more suitable explanation of the large structure in germanium is required.

The predictions of isospin formalism, whlle they are useful in the $(\gamma, n p)$ and $(\gamma, p)$ cross section analysis, provlde little aid in explaining the total cross sections. The maximum splitting of the two isospin components of the giant resonance is predicted to be 5 to $6 \mathrm{MeV}$ for the germanlum isotopes, much less than the energy separation of the two prominent resonances observed in the total cross sections. Isospin formalism predicts that splltting will increase as $T_{0}$ increases, In direct opposition to the decrease in separation energy observed in the experiment results. Isospin sum rule calculations predict an integrated cross section for the $T_{>}$resonance that is a maximum of $23 \%$ of the $T_{<}$resonance, and that the $T_{>}$strength should systematically decrease as. $T$ increases. Again this prediction is quite opposite from the observed trends in this experiment. In fact, we have al ready identified the $T_{\text {, contributions in }}$ the $(\gamma, n p)$ and $(\gamma, p)$ results within the primary resonance and have no

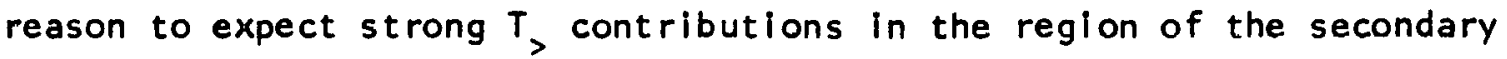
resonance.

A third possible mechanism to account for structure above. the giant resonance is deformation splitting of the glant resonance, as predicted by hydrodynamic models of dipole absorption. This explanation is unlikely 
to be of use in germanium for several reasons; in particular, there are no indications of signiflcant ground state deformation in the spectra of these nuclei. The low lying energy levels in each nucleus can be accounted for on the basis of shell model configurations without the use of deformation. Measured $B(E 2)$ values (54) indicate some evidence of slight deformation but not an effect large enough to produce the large resonance separations observed here. Indeed, a survey of experimental results for nuclei with large deformations (74) suggest $5 \mathrm{MeV}$ as the maximum resonance splitting produced by deformation. The observed ratio of integrated cross section is roughly $2: 1$ with few exceptions $(74)$, the second peak belng the larger in area. This result is opposite to ratio of cross sections observed in germanium.

We are left with considering the final possible mechanism, additional dipole contributions. The possibllity of dipole overtone resonances has been suggested, but the predicted energy of the first overtone lies at about $54 \mathrm{MeV}$ in germanium, much above the energy of the second resonance. it is doubtful that dipole overtones can account for the structures seen in germanium.

It is of great interest to note that this pronounced second resonance may occur in nuclel over a wide range of nuclear mass. Berman et al. (7) report structure above the giant resonance in the total photoneutron cross sections of ${ }^{75} \mathrm{As},{ }^{107} \mathrm{Ag}$ and ${ }^{138} \mathrm{C}$. Recent electron scattering experiments (75) have Identified high energy structures $\ln { }^{90} \mathrm{Zr}$ and other elements. While the reported strengths are not as great as that seen in germanium, the comparison verifies that this structure may not be unusual. 
A particularly convincing parallel exists in the total photoneutron cross sections of tin isotopes reported by fultz et al. (27). In this work considerable strength is reported above the main giant resonance. To provide a basis for comparison with the germanium results, we have fit the cross sections to Lorentz line shapes in the same manner as the germanlum cross sectlons. The results of the fitting procedure are given In Table 10. Figure 22 compares the cross sections to the fitted curves. The strength above the glant resonance in $t$ in is located near the predicted energies of the $T_{>}$resonance and the quadrupole resonance. However, the integrated cross section exceeds the sum of the predicted T, and quadrupole contrlbutions by a factor of two. Since fultz et al. could not separate the $(\gamma, n)$ and $(\gamma, n p)$ contributions, they assign the excess strength to the $(\gamma, n p)$ cross section. This explanation now seems inadequate in light of the germanium results for the $(\gamma, n p)$ contribution. In germanium the $(\gamma, n p)$ cross section is much too small to produce any considerable change in the total cross section. The comparison of tin and germanium is even more convincing if one examines the fitted parameters for tin listed in Table 11. The behavior of the second resonance in tin is remarkably like that of germanium. The width of the secondary resonance is considerably larger than the main resonance width in all isotopes except ${ }^{116} \mathrm{Sn}$. The energy of the second resonance decreases with increasing neutron excess for all isotopes except ${ }^{124} \mathrm{Sn}$. The complete comparison of tin results to germanium (Tables 8 and 9) leaves little doubt that the maln body of structure in the secondary resonance of both experiments result from the same mechanism. 
Table 10. Resonance strengths for tin

\begin{tabular}{|c|c|c|c|c|c|c|}
\hline Nucleus & $\begin{array}{c}\mathrm{S}_{1} \\
(\mathrm{MeV}-\mathrm{mb})\end{array}$ & $\begin{array}{c}\mathrm{S}_{2} \\
(\mathrm{MeV}-\mathrm{mb})\end{array}$ & $\begin{array}{c}\mathrm{S}_{\mathrm{TOT}} \\
(\mathrm{MeV}-\mathrm{mb})\end{array}$ & $s_{2} / s_{1}$ & $\begin{array}{l}60 \mathrm{NZ} / \mathrm{A} \\
(\mathrm{MeV}-\mathrm{mb})\end{array}$ & $\mathrm{S}_{1} / 60 \mathrm{NZ} / \mathrm{A}$ \\
\hline${ }^{116} \mathrm{sn}$ & 1631 & 139 & 1770 & .085 & 1707 & 0.96 \\
\hline $118_{\mathrm{Sn}}$ & 1736 & 271 & 2007 & .156 & 1728 & 1.01 \\
\hline${ }^{120} \mathrm{Sn}$ & 1915 & 315 & 2230 & .141 & 1750 & 1.10 \\
\hline${ }^{124} \mathrm{Sn}$ & 1868 & 272 & 2140 & .145 & 1790 & 1.04 \\
\hline
\end{tabular}

Table 11. Fitted parameter of tin cross sections

\begin{tabular}{lcccccc}
\hline Nucleus & $\begin{array}{c}\sigma_{a}^{0} \\
(\mathrm{mb})\end{array}$ & $\begin{array}{c}\Gamma_{a} \\
(\mathrm{MeV})\end{array}$ & $\begin{array}{c}E_{a} \\
(\mathrm{MeV})\end{array}$ & $\begin{array}{c}\sigma_{b}^{0} \\
(\mathrm{mb})\end{array}$ & $\begin{array}{c}\Gamma_{b} \\
(\mathrm{MeV})\end{array}$ & $\begin{array}{c}E_{b} \\
(\mathrm{MeV})\end{array}$ \\
\hline $116_{\mathrm{Sn}}$ & 270 & 4.29 & 15.6 & 19.6 & 2.10 & 27.6 \\
$118_{\mathrm{Sn}}$ & 255 & 4.87 & 15.5 & 29.1 & 6.98 & 24.0 \\
$120 \mathrm{Sn}$ & 280 & 4.90 & 15.4 & 35.0 & 6.67 & 23.0 \\
$124 \mathrm{Sn}$ & 285 & 4.68 & 15.18 & 28.9 & 7.08 & 25.0 \\
\hline
\end{tabular}



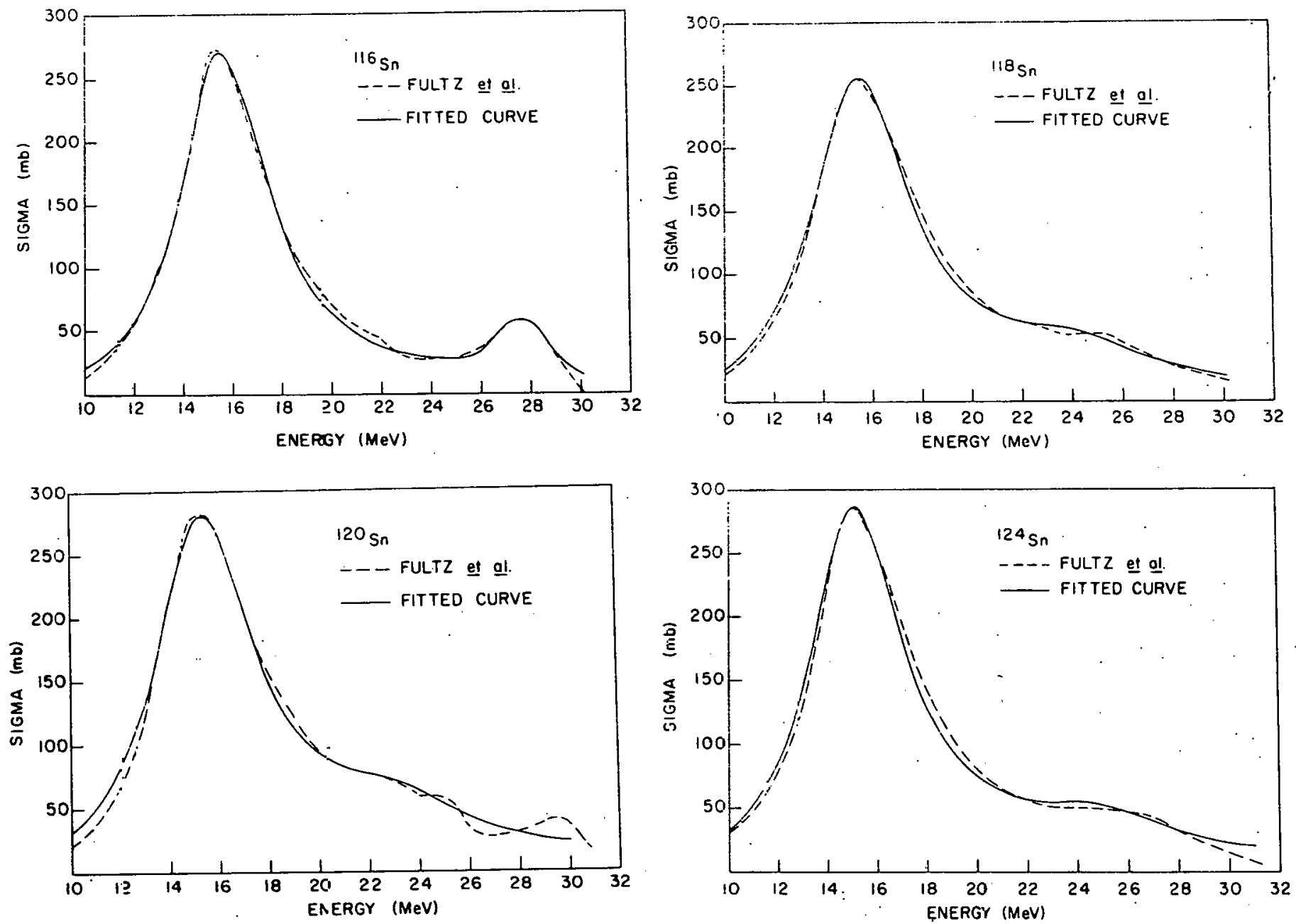

Figure 22. Total neutron cross sections of ${ }^{116} \mathrm{Sn},{ }^{118} \mathrm{Sn},{ }^{120} \mathrm{Sn}$ and ${ }^{124} \mathrm{Sn}$ from Fultz et al. 
We conclude on the basis of the above discussion that structure above the glant resonance that has definite systematic energy dependence and considerable cross section strength is well established in experimental results. Also, a suitable interaction mechanism to explain this structure has not yet been put forward.

\section{Suggestions for Future Work}

The medium mass region of the periodic table has not been well studied. In particular, few measurements exist above $30 \mathrm{MeV}$, and still fewer measure anything except $(\gamma, n)$ and possibly $(\gamma, 2 n)$ cross sections. The present measurements of germanium cross sections have revealed structure which is not easlly explained by current models of the nuclear photo-effect. This strongly suggests that simllar systematic studies of medium mass nuclei should be performed. In particular, experiments that measure as many decay channels as possible, or that they are sensitive to the total absorption cross section itself, would be most valuable in characterizing high energy structures. In the case of several nuclei (Se, $\mathrm{Kr}, \mathrm{Zr}$ and Mo) activation measurements (in the manner presented in this experiment) could be combined with direct neutron counting experiments to give total cross sections. In particular, the $(\gamma, p)$ and $(\gamma, n p)$ cross sections for tin could be studled by activation and be combined with the results of Fultz. The advent of the new high intensity linac at MIT, will provide an opportunity to study these effects with inelastic electron scattering experiments. Using separated isotopes, many nuclei could be studied not available to activation studies.

There are several measurements which would be of immediate interest 
106

in light of results reported here. A more complete verification of isospin sum rule calculations could be obtained by measuring the ${ }^{70} \mathrm{Ge}(\gamma, p)$, ${ }^{72} \mathrm{Ge}(\gamma, \mathrm{p}),{ }^{73} \mathrm{Ge}(\gamma, \mathrm{p})$ and ${ }^{76} \mathrm{Ge}(\gamma, \mathrm{p})$ reaction cross sections. The measure-

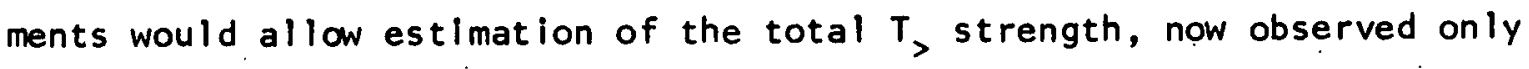
In the $(\gamma, n p)$ channel. In addition, the ${ }^{73} \mathrm{Ge}(\gamma, p)$ measurement would complete the $(\gamma, n p)$ measurements by allowing a separation of the present ${ }^{74} \mathrm{Ge}(\gamma, \mathrm{np})+{ }^{73} \mathrm{Ge}(\gamma, \mathrm{p})$ results and help identify systematic structure in the np channel. The ${ }^{76} \mathrm{Ge}(\gamma, \mathrm{p})$ could be measured in a simple activation experiment, and the others by direct proton counting using separated isotopes as targets. In ${ }^{70} \mathrm{Ge}$ and ${ }^{72} \mathrm{Ge}$ the inverse $(p, \gamma)$ cross sections could also be used instead of the $(\gamma, p)$ result. 


\section{REFERENCES}

1. M. Goldhaber and E. Teller, Phys. Rev. 74, 1046 (1948).

2. D. H. Wilkinson, Physica 22, 1039 (1956).

3. E. P. Wigner, Phys. Rev. 51, 706 (1937).

4. J. E. Anderson, C. Wong, and.J. W. McClure, Phys. Rev. 126, 2170 (1962).

5. G. C. Baldwin and G. S. Klaiber, Phys. Rev. 73, 1156 (1948).

6. S. C. Fultz, R. L. Bramblett, J. T. Caldwell, and N. A. Derr, Phys. Rev. 136, B126 (1964).

7. B. L. Berman, R. L. Bramblett, J. T. Caldwell, H. S. Davis, M. A. Kelly, and S. C. Fultz, Phys. Rev. 177, 1745 (1969).

8. C. D. Bowman, G. F. Auchampaugh, and S. C. Fultz, Phys. Rev. 133B, $676(1964)$.

9. B. L. Berman, M. A. Kelly; R. L. Bramblett, J. T. Caldwell, H. S. Davis, and S. C. Fultz, Phys. Rev. 185, 1576 (1969).

10. E. G. Fuller and Evans Hayward, Nuclear Physics 30, 613 (1962).

11. L. Katz and R. Montalbetti, Can. J. Phys. 31, 798 (1953).

12. B. M. Spicer, Phys. Rev. 99, 33 (1955).

13. A. S. Penfold and B. M. Spicer, Phys. Rev. 100, 1377 (1955):.

14. S. A. E. Johansson and B. Forkman, Arkiv. Fysik 12, 359 (1957).

15. P. S. Flsher, U. F. Measday, F. A. Nikolaev, A. Kalmykov, and

A. B. Clegg, Nuclear Physics 45, 113 (1963).

16. P. P. Singh, R. E. Segel, L. Meyer-Schutzmeister, S. S. Hanna, and R. G. Allas, Nuclear Physics 65,577 (1965).

17. D. M. Brink, Nuclear Physics 4, 215 (1957).

18. V. V. Balashov, Sov. Phys. JETP 42, 275 (1962).

19. R. G. Johnson, Ph.D. thesis, lowa State University, Ames, lowa, 1970 (unpubIIshed). 
20. R. E. Segel, in Isobaric Spin in Nuclear Physics, edited by $J$. $D$. Fox and D. Robson (Academic Press Tnc., New York, 1966.). F. 194.

21. P. Axe1, D. M. Drake, S. Whetstone, and S. S. Hanna, Phys. Rev. Letters 19, 1343 (1967).

22. R. 0. Akyuz and Fallleros, Phys. Rev. Letters 27, 1016 (1971).

23. K. Shoda, M. Sugawara, T. Saito, and H. Miyase, Phys. Rev. Letters 23, $800(1969)$.

24. B. L. Berman, J. T. Caldwell, R. R. Harvey, M. A. Kelly, R. L. Bramblett, and S. C. Fultz, Phys. Rev. 162, 1098 (1967).

25. H. Arenhovel and J.. M. Maison, Nucl. Phys. Al47, 305 (1970).

26. K. Min, Phys. Rev. 182, 1359 (1969).

27. S. C. Fultz, R. L. Bramblett, B. L. Berman, J. T. Caldwell, and M. A. Kelly, Phys. Rev. 186, 1255 (1969).

28. F. H. Schamber, Ph.D. thesis, lowa State University, Ames, lowa, 19.70 (unpublished).

29. P. Paul, J. F. Amann, and K. A. Snover, Phys. Rev. Letters 27 , 1013 (1971).

30. G. E. Clark, Ph.D. thesis, lowa State University, Ames, lowa, 1972 (unpublished).

31. H. J. Vander Molen, Ph.D. thesis, lowa State University, Ames, lowa, 1972 (unpublished).

32. J. S. Levinger and H. A. Bethe, Phys. Rev. 78, 155 (1950).

33. J. Le Tourneaux, Phys. Letters 13, 325 (1964).

34. M. Danos and W.'Greiner, Phys. Rev. 134, B284 (1964).

35. D. S. Fielder, J. Le Tourneaux, K. Min, and W. D. Whitehead, Phys. Rev. Letters 15, 33 (1965).

36. J. P. Elliott and B. H. Flowers, Proc. Roy. Soc. (London) A242, 57 (1957).

37. L. E. Trainor, Phys. Rev. 85, 962 (1952).

38. L. A. Radicati, Phys. Rev. 87, 521 (1952). 
39. M. Gell-Mann and V. L. Telegdi, Phys. Rev. 91, 169 (1963).

40. R. Leonardi, Phys. Rev. Letters 28, 836 (1972).

41. B. Goulard and S. Fallieros, Can. J. Phys. 45, 322 (1967).

42. J. S. O'Connel1, Phys. Rev. Letters 22, 1314 (1969).

43. E. Hayward, B. F. Gibson, and J. S. O'Connell, Phys. Rev. 5C, $846(1972)$.

44. J. Jannecke, Nucl. Phys. 73,97 (1965).

45. J. E. Grlffin and C. L. Hammer, U.S. Atomic Energy Commission Report. 1S-676, 1963 (unpublished).

46. J. S. Pruitt and S. R. Domen, National Bureau of Standards Monograph $48,1962$.

47. L. 1. Schiff, Phys. Rev. 83, 252 (1951).

48. M. A. Mariscotti, Nucl. Instr. Meth. 50, 309 (1967).

49. E. Bramanis, T. K. Deague, R. S. Hicks, R. J. Hughes, E. G. Mui rhead, R. H. Sambell, and R. J. J. Stewart, Nucl, Instr. Meth. 100, 59 (1972).

50. L. Katz and A. G. W. Cameron, Can. J. Phys. 29, 518 (1963).

51. A. S. Penfold and J. E. Leiss, University of Illinois Report (1959) (unpublished).

52. B. C. Cook, Nucl. Instr. Meth. 24, 256 (1963).

53. C. M. Lederer, J. M. Hollander, and I. Pulman, Table of Isotopes, Sixth Edition, (John Wiley and Sons, New York, 1967.)

54a. Nuclear Data Sheets, Nuclear Data Group, Oak Ridge Natl. Laboratory, Academic Press, Inc., New York. B2-6, 93, 111 (1968).

54b. Nuclear Data Sheets, Nuclear Data Group, Oak Ridge Natl, Laboratory, Academic Press, Inc., New York, Bl-6, 1, 27, 59, 103 (1966).

55. K. A. Baskova, S. S. Vasllev, M. A. Khamo-Lella, L. Y. Shautualov, Bull. Acad. Sci. USSR, Phys. Ser. 29, 201 (1966).

56. F. R. Hudson, P. F. Bampton, R. N. Glover, Phys. Letters 27B, $84(1968)$. 
57. H. Langhoff, L. Frevert, Nucl. Phys. All1, 225 (1968).

58. A. W. Schardt, J. P. Welker, Phys. Rev. 99, 810.(1955).

59. H. J. van den Bold, H. C. Geijn, P. M. Endt, Physica 24, 23 (1958).

60. C. Ythier, R. K. Girgis, R. A. Ricci, R. van Lieshout, Nucl. Phys. 9. 108 (1958).

61. F. Schweizer, G. W. Eaken, J. Van Klinken, Bull. Am. Phys. Soc. I1, No. 4, 458, Bc7 (1966).

62. M. E. Bunker, J. P. Mize, J. W. Starner, Phys. Rev. 105, 227 (1957).

63. D. E. Alburger, Phys. Rev. 199, 1222 (1958).

64. L. M. Langer, D. R. Smith, Phys. Rev. 119, 1308 (1960).

65. V. D. Vitman, N. A. Voinova, B. S. Dzhelepov, Nucl. Phys. 46,368 (1963).

66. E. Elchler, G. D. O'Kelly, R. L. Robinson, J. A. Marinsky, N. R. Johnson, Nucl. Phys. 35, 625 (1962).

67. 0. A. Borello, J. Goldenberg, and M. D. S. Santos, Am. Acad. Brasil. Cienc. 27, 413 (1955).

68. F. Ferrero, S. Ferroni, R. Malvano, S. Menardi, and E. Silva, Nucl. Phys. 1.5, 436 (1960).

69. L. Katz and G. W. Cameron, Can. J. Phys. 29, 518 (1951).

70. D. G. Owen and E. G. Muirhead, private communication (1972).

71. V. G. Shevchenko, N. P. Yudin, B. A. Yurlev, JETP Lett. 45,180 (1963).

72. A. Lushnikov and M. Urin, Sov. J. Nucl. Phys. 1, 311 (1965).

73. R. Ligensa, W. Greiner, and M. Danos, Phys. Rev. Letters, 16, $364(1966)$.

74. E. Hayward, in Photonuclear Reactions, NBS Monograph 118, (1870).

75. S. Fukuda and Y. Torlzuka, Phys. Rev. Letters, 29, 1109 (1972). 


\section{ACKNOWLEDGMENTS}

I wish to thank the many people whose support contributed so much to this effort. In particular, I wish to thank my major advisor, Dr. R. C. Morrison for his encouragement, support and guidance throughout the entire project. Special gratitude is due also to Dr. D. J. Zaffarano who, has served as an inspiration during my graduate years at lowa state. The help provided by many other people has been invaluable, and deserves acknowledgment here: Dr. J.E.E. Baglin for his encouraging and helpful suggestions, Dr. A. J. Bureau and Mr., George Holland for their constant attention to synchrotron operation which allowed us to perform the experiment, Dr. Gary Clark, Dr. Harold Vander Molen, Mr. Bob Pritchard, and Mr. David Fromme for their careful help in collecting data. Special thanks go to Dr. W. L. Talbert, Jr., and Dr. W. C. Schick, Jr., and their groups for the loan of the Ge(Li) detectors used in this experiment.

I especially wish to thank my wife, Joan, for her love and understanding which have been a great source of strength for me. Her contribution to this project is easily as large as that of those mentioned above. 